\title{
Estimates by gap potentials of free homotopy decompositions of critical Sobolev maps
}

https://doi.org/10.1515/anona-2020-0047

Received November 28, 2018; accepted October 22, 2019.

Abstract: A free homotopy decomposition of any continuous map from a compact Riemannian manifold $\mathcal{M}$ to a compact Riemannian manifold $\mathcal{N}$ into a finite number maps belonging to a finite set is constructed, in such a way that the number of maps in this free homotopy decomposition and the number of elements of the set to which they belong can be estimated a priori by the critical Sobolev energy of the map in $W^{s, p}(\mathcal{M}, \mathcal{N})$, with $s p=m=\operatorname{dim} \mathcal{M}$. In particular, when the fundamental group $\pi_{1}(\mathcal{N})$ acts trivially on the homotopy group $\pi_{m}(\mathcal{N})$, the number of homotopy classes to which a map can belong can be estimated by its Sobolev energy. The estimates are particular cases of estimates under a boundedness assumption on gap potentials of the form

$$
\iint_{\substack{(x, y) \in \mathcal{M} \times \mathcal{M} \\ d_{\mathcal{N}}(f(x), f(y)) \geq \varepsilon}} \frac{1}{d_{\mathcal{M}}(y, x)^{2 m}} \mathrm{~d} y \mathrm{~d} x .
$$

When $m \geq 2$, the estimates scale optimally as $\varepsilon \rightarrow 0$. When $m=1$, the total variation of the maps appearing in the decomposition can be controlled by the gap potential. Linear estimates on the Hurewicz homomorphism and the induced cohomology homomorphism are also obtained.

Keywords: Free homotopy; Hurewicz homomorphism; vanishing mean oscillation (VMO); fractional Sobolev spaces

MSC: 46E35 (55M25, 55P99, 55Q25, 58A12

\section{Introduction}

\subsection{Estimates on the topological degree}

Brouwer's topological degree classifies the homotopy classes of continuous maps from a sphere $\mathbb{S}^{m}$ to itself. If the map $f \in \mathscr{C}^{1}\left(\mathbb{S}^{m}, \mathbb{S}^{m}\right)$ is smooth, then its degree $\operatorname{deg}(f)$ can be computed by the classical Kronecker formula

$$
\operatorname{deg}(f)=\int_{\mathbb{S}^{m}} f^{\star} \omega_{\mathbb{S}^{m}},
$$

where $\omega_{\mathbb{S}^{m}}$ is the volume form on the sphere $\mathbb{S}^{m}$ normalized so that $\int_{\mathbb{S}^{m}} \omega_{\mathbb{S}^{m}}=1$ and $f^{\star} \omega_{\mathbb{S}^{m}}$ is the pullback of the form $\omega_{\mathbb{S}^{m}}$ by the map $f$. In view of the classical inequality between the geometric and quadratic means, we have

$$
\left|f^{\star} \omega_{\mathbb{S}^{m}}\right|=|\operatorname{det} D f| \omega_{\mathbb{S}^{n}} \leq \frac{|D f|^{m}}{m^{m / 2}} \omega_{\mathbb{S}^{m}}
$$

everywhere on the sphere $\mathbb{S}^{m}$; this implies then the integral estimate on the degree (see [4, Remark 0.7])

$$
|\operatorname{deg}(f)| \leq \frac{1}{m^{m / 2}} f_{\mathbb{S}^{m}}|D f|^{m}=\frac{1}{m^{m / 2}\left|\mathbb{S}^{m}\right|} \varepsilon^{1, m}(f),
$$

\footnotetext{
*Corresponding Author: Jean Van Schaftingen, Université catholique de Louvain (UCLouvain), Institut de Recherche en Mathématique et Physique (IRMP), Chemin du Cyclotron 2 bte L7.01.01, 1348 Louvain-la-Neuve, Belgium, E-mail: Jean.VanSchaftingen@uclouvain.be
} 
where we have defined the Sobolev energy $\mathcal{E}^{1, p}$ for $p \in[1,+\infty)$ by

$$
\mathcal{E}^{1, p}(f) \triangleq \int_{\mathbb{S}^{m}}|D f|^{p}
$$

This estimate (1.2) remains valid under the weaker assumption that the map $f$ lies in the Sobolev space $W^{1, m}\left(\mathbb{S}^{m}, \mathbb{S}^{m}\right)$ of weakly differentiable maps whose weak derivative satisfies the integrability condition that $\int_{\mathbb{S}^{m}}|D f|^{n}<+\infty$; the degree of $f$ is then understood in the sense of maps of vanishing mean oscillation (VMO) [11]. By the classical Hölder inequality, the estimate (1.2) implies that the degree of $f$ can also be controlled by the $L^{p}$ norms of its derivative $D f$ for $p \in(m,+\infty]$.

Although the integral formula (1.1) does not have a clear sense when the map $f$ does not have some kind of derivatives, the natural counterpart of the integral estimate (1.2) still holds for fractional Sobolev maps: for every $p \in(m,+\infty)$, there exists a constant $C_{m, p}$ such that for every map $f \in W^{m / p, p}\left(\mathbb{S}^{m}, \mathbb{S}^{m}\right)$, one has [4, theorem 0.6] (see also [theorem A.3; 6; 33, theorem 2.3])

$$
|\operatorname{deg}(f)| \leq C_{m, p} \varepsilon^{m / p, p}(f),
$$

where the fractional Sobolev energy of the map $f: \mathbb{S}^{m} \rightarrow \mathbb{S}^{m}$ is defined for any $s \in(0,1)$ and $p \in[1,+\infty)$ as

$$
\mathcal{E}^{s, p}(f) \triangleq \iint_{\mathbb{S}^{m} \times \mathbb{S}^{m}} \frac{|f(y)-f(x)|^{p}}{|y-x|^{m+s p}} \mathrm{~d} x \mathrm{~d} y,
$$

and the finiteness of the quantity $\mathcal{E}^{s, p}(f)$ is equivalent to have the map $f$ belonging to the fractional Sobolev space $W^{s, p}\left(\mathbb{S}^{m}, \mathbb{S}^{m}\right)$ with $s=m / p$.

Although the degree is naturally defined for continuous maps or for maps of vanishing mean oscillation (VMO), the associated norms in $L^{\infty}$ or in BMO remain bounded over the entire class of maps on the sphere and cannot control in any way the topology,

Jean Bourgain, Haïm Brezis, Petru Mironescu and NGuYÊN Hoài-Minh have obtained gap potential estimates of the degree that imply integral estimates of the form (1.2) and (1.3) [4, open problem 2; 5, theorem $1.1 ; 37$ ] (see also [42]): they have proved that for every $\varepsilon \in\left(0, \sqrt{2\left(1+\frac{1}{m+1}\right)}\right)$, there exists a constant $C_{\varepsilon, m}$ such that for every map $f \in \mathscr{C}\left(\mathbb{S}^{m}, \mathbb{S}^{m}\right)$, one has

$$
|\operatorname{deg}(f)| \leq C_{\substack{(x, y) \in \mathbb{S}^{m} \times \mathbb{S}^{m} \\|f(y)-f(x)|>\varepsilon}} \frac{1}{|y-x|^{2 m}} \mathrm{~d} x \mathrm{~d} y .
$$

In view of the definition (1.4), the gap potential estimate (1.5) implies the fractional Sobolev estimate (1.3). If $m \geq 2$, the constant can be taken to satisfy $C_{\varepsilon, m} \leq C_{m} \varepsilon^{m}$ [43].

The gap potentials on the right-hand side of (1.5) also appeared in estimates on lifting of maps into the circle [39, theorem 2] and were showed to characterize as $\varepsilon \rightarrow 0$ Sobolev spaces $[35,36,38,44]$ and to provide a property stronger than VMO [10].

\subsection{Estimates on the Hopf invariant}

Continuous maps from $\mathbb{S}^{3}$ into $\mathbb{S}^{2}$ are classified by the Hopfinvariant $\operatorname{deg}_{H}(f)$. Tristan RIviÈRE has proved that [49, lemma III.1] (see also [20; 21, Lemma 7.12])

$$
\left|\operatorname{deg}_{H} f\right| \leq C\left(\int_{\mathbb{S}^{3}}|D f|^{3}\right)^{1+\frac{1}{3}} .
$$

Compared to the corresponding estimate of the topological degree (1.2), a power $1+\frac{1}{3}$ applied to the integral appears, related to the Whitehead formula for the Hopf invariant [58]. No fractional counterpart to (1.6) seems to be know (see open problem 5 below). 
Rivière's bound (1.6) extends straightforwardly to its higher-dimensional counterpart which is a homotopy invariant for maps from the sphere $\mathbb{S}^{2 n-1}$ into $\mathbb{S}^{n}$ [3, proposition 17.22], resulting in an estimate

$$
\left|\operatorname{deg}_{H} f\right| \leq C\left(\int_{\mathbb{S}^{2 n-1}}|D|^{2 n-1}\right)^{1+\frac{1}{2 n-1}} .
$$

The Hopf invariant takes nontrivial values when $n$ is even [3, proposition 17.22] but is not necessarily injective (when $n \in\{1, \ldots, 20\}$, it is injective if and only if $n \in\{2,6\}[56]$ ). In all cases, only finitely many homotopy classes share the same value of the Hopf invariant and thus any set of maps which is bounded in the Sobolev space $W^{1,2 m-1}\left(\mathbb{S}^{m}, \mathbb{S}^{2 m-1}\right)$ is contained in finitely homotopy classes of maps.

By a theorem of Jean-Pierre SERRE [55], all the other classes of continuous maps between spheres of different dimension consist only of finitely many homotopy classes; thus in general a bounded set of maps in $W^{1,3}\left(\mathbb{S}^{m}, \mathbb{S}^{n}\right)$ is contained in finitely many homotopy classes.

\subsection{Estimates on free homotopy decompositions}

The results outlined above for maps between spheres raise the question whether sets which are bounded Sobolev norms are contained in finitely many homotopy classes of maps.

When $s \in(0,1), p \in(1,+\infty)$ and $s p>m$, the classical Morrey-Sobolev embedding (see for example [7, theorem 9.12; 59, lemma 6.4.3]) ensures that sets which are bounded in energy in $W^{s, p}\left(\mathbb{S}^{m}, \mathcal{N}\right)$ are also bounded in the space of Hölder-continuous functions $\mathscr{C}^{0, s-m / p}\left(\mathbb{S}^{m}, \mathcal{N}\right)$, and thus by the Ascoli compactness criterion and the local invariance of homotopy classes, they are contained in finitely homotopy classes.

A slightly more subtle case is $W^{1,1}\left(\mathbb{S}^{1}, \mathcal{N}\right)$ : although there is no compact embedding in the set of continuous maps, each map is homotopic to a map whose Lipschitz constant is controlled; hence bounded sets are contained in finitely many homotopy classes.

In the general case of $W^{s, p}\left(\mathbb{S}^{m}, \mathcal{N}\right)$ with $s p=m, p>1$ and an arbitrary target manifold $\mathcal{N}$, such a control turns out to be impossible. In order to construct infinitely many non-homotopic maps whose Sobolev energies remain bounded, we rely on the following definition:

Definition 1.1 (Free homotopy decomposition). A map $f \in \mathscr{C}\left(\mathbb{S}^{m}, \mathcal{N}\right)$ has a free homotopy decomposition into the maps $f_{1}, \ldots, f_{k} \in \mathscr{C}\left(\mathbb{S}^{m}, \mathbb{S}^{m}\right)$ whenever there exists a map $g \in \mathscr{C}\left(\mathbb{S}^{m}, \mathcal{N}\right)$ homotopic to $f$ on $\mathbb{S}^{m}$ and nontrivial geodesic balls $B_{\rho_{1}}\left(a_{1}\right), \ldots, B_{\rho_{\ell}}\left(a_{k}\right) \subset \mathbb{S}^{m}$ such that $g$ is constant on $\mathbb{S}^{m} \backslash \bigcup_{i=1}^{k} B_{\rho_{i}}\left(a_{i}\right)$ and for every $i \in\{1, \ldots, k\}$, its restriction $\left.g\right|_{\bar{B}_{\rho_{i}}\left(a_{i}\right)}$ is homotopic to some $f_{i} \in \mathscr{F}$ on $\mathbb{S}_{m} \simeq \bar{B}_{\rho_{i}}\left(a_{i}\right) / \partial B_{\rho_{i}}\left(a_{i}\right)$.

The map $g$ is well defined on the quotient $\bar{B}_{\rho_{i}}\left(a_{i}\right) / \partial B_{\rho_{i}}\left(a_{i}\right) \simeq \mathbb{S}^{m}$ because it is constant on $\partial B_{\rho_{i}}\left(a_{i}\right)$.

The free homotopy decomposition appears in the construction of harmonic and polyharmonic maps that are known in many instances to generate through free homotopy decomposition all the homotopy classes [18, theorem 14; 50, theorem 5.5].

The free homotopy decomposition is an invariant under homotopies of the maps, but is not in general a faithful invariant: for example if $\mathcal{N}=\left(\mathbb{S}^{1} \times \mathbb{S}^{2 m} \cup \mathbb{S}^{m} \times \mathbb{S}^{m+1}\right) / \mathbb{S}^{2 m}$, then there are two maps into which infinitely many homotopy classes decompose freely (see proposition 2.4 below).

The next result shows that maps that have the same free homotopy decomposition satisfy up to homotopy the same fractional Sobolev bound.

Theorem 1.2 (Bound on the Sobolev energy by free homotopy decomposition). Let $m \in \mathbb{N}_{\star}$ and $\mathcal{N}$ be a connected Riemannian manifold. If $f \in \mathscr{C}\left(\mathbb{S}^{m}, \mathcal{N}\right)$ has a free homotopy decomposition into $f_{1}, \ldots, f_{k} \in \mathscr{C}\left(\mathbb{S}^{m}, \mathcal{N}\right)$, then for every $s \in(0,1]$ and every $p \in[m,+\infty)$ such that $p=m / s>1$, one has

$$
\inf \left\{\mathcal{E}^{s, p}(g) \mid g \in\left(\mathscr{C} \cap W^{s, p}\right)\left(\mathbb{S}^{m}, \mathcal{N}\right) \text { is homotopic to } f\right\} \leq \sum_{i=1}^{k} \mathcal{E}^{s, p}\left(f_{i}\right) .
$$


In particular, theorem 1.2 implies that all the homotopy classes that decompose freely into the maps $f_{1}, \ldots, f_{k}$ satisfy the same energy bound; if there are infinitely many such homotopy classes, then there are infinitely many nonhomotopic map satisfying the same energy bound.

The proof of theorem 1.2 is performed by gluing together the maps $f_{1}, \ldots, f_{k}$ with an arbitrarily small energetic cost of gluing, performed through conformal transformations by Mercator projections. Theorem 1.2 does not cover the case $s=p=m=1$. This is consistent with our observation that a Sobolev energy bound gives a control on the homotopy classes.

By taking the phenomenon described in theorem 1.2 into account, it has been proved that for every $\lambda>0$, there exists a finite set $\mathscr{F}$ and $k \in \mathbb{N}$ such that every map $f \in\left(W^{s, p} \cap \mathscr{C}\right)\left(\mathbb{S}^{m}, \mathcal{N}\right)$ satisfying $\mathcal{E}^{s, m / s}(g) \leq \lambda$ has a free homotopy decomposition into $k$ maps of the set $\mathscr{F}$ for $m=1, s=\frac{1}{2}$ and $p=2$ by Ernst KuWERT [30], when $m \geq 1, s=1$ by Frank DUZAAR and Ernst KUWERT [14, theorem 4], when $m \geq 1$ and $s=1-\frac{1}{m+1}$ by Thomas MÜLLER [34, theorem 5.1] and when $m=2$ and $s=1$ by Richard SCHOEN and Jon WoLFSON [54, lemma 5.2].

The critical case $s p=m$ for estimates can be seen as a limiting case between the classical continuous picture of homotopy classes in the supercritical $s p>m$ and the combination of collapses and appearance of homotopy classes in the subcritical case $s p<m[8,9,23-26,57]$.

Our main result shows that these estimates are in fact consequences of a stronger gap potential estimate similar to (1.5).

Theorem 1.3 (Free homotopy decompositions controlled by a gap potential). Let $m \in \mathbb{N} \star$ and $\mathcal{N}$ be a compact Riemannian manifold. If $\varepsilon>0$ is small enough, then there is a constant $C>0$ such that for every $\lambda>0$, there exists a finite set $\mathscr{F}^{\lambda} \subset \mathscr{C}\left(\mathbb{S}^{m}, \mathcal{N}\right)$ such that any map $f \in \mathscr{C}\left(\mathbb{S}^{m}, \mathcal{N}\right)$ satisfying

$$
\iint_{\substack{(x, y) \in \mathbb{S}^{m} \times \mathbb{S}^{m} \\ d_{\mathcal{N}}(f(y), f(x))>\varepsilon}} \frac{1}{|y-x|^{2 m}} \mathrm{~d} y \mathrm{~d} x \leq \lambda,
$$

has a free homotopy decomposition into $f_{1}, \ldots, f_{k} \in \mathscr{F}^{\lambda}$ with $k \leq C \lambda$.

In fact it can be observed that under the assumptions of theorem 1.3 any measurable map that satisfies the integrability condition with $\varepsilon$ small enough has a small mean oscillation on small scales [10, proposition 1; 41] and therefore can be associated naturally and uniquely to a homotopy class of continuous maps from $\mathbb{S}^{m}$ to $\mathcal{N}$ (see [11, (8), remark 7 and lemma A.5]).

The appearance of free homotopy decompositions in which the way of gluing the $k$ maps together is arbitrary and uncontrolled can be thought of as a topological bubbling phenomenon, which is a topological version of the geometric bubbling phenomenon in conformally invariant geometric problems $[13,45,50]$. In many cases however, theorem 1.3 implies that maps satisfying a bound on the gap potential can only belong to finitely many homotopy classes.

Theorem 1.4 (Finitely many homotopy classes under a gap potential bound). Let $m \in \mathbb{N} \star$ and $\mathcal{N}$ be a compact Riemannian manifold. If $m=1$ and every conjugacy class of $\pi_{1}(\mathcal{N})$ is finite or $m \geq 2$ and every orbit of the action of $\pi_{1}(\mathcal{N})$ on $\pi_{m}(\mathcal{N})$ is finite, and if $\varepsilon>0$ is small enough, then for every $\lambda>0$, there exists a finite set $\mathscr{G}^{\lambda} \subset \mathscr{C}\left(\mathbb{S}^{m}, \mathcal{N}\right)$ such that any map $f \in \mathscr{C}\left(\mathbb{S}^{m}, \mathcal{N}\right)$ satisfying

$$
\iint_{\substack{(x, y) \in \mathbb{S}^{m} \times \mathbb{S}^{m} \\ d_{\mathcal{N}}(f(y), f(x))>\varepsilon}} \frac{1}{|y-x|^{2 m}} \mathrm{~d} x \mathrm{~d} y \leq \lambda,
$$

is homotopic to some $\mathrm{g} \in \mathscr{G}^{\lambda}$.

The assumptions of theorem 1.4 are satisfied in particular when the fundamental group $\pi_{1}(\mathcal{N})$ is finite, if $m=1$ and $\pi_{1}(\mathcal{N})$ is abelian or if $m \geq 2$ and the action of $\pi_{1}(\mathcal{N})$ on the homotopy group $\pi_{m}(\mathcal{N})$ is trivial.

In particular, under the assumptions of theorem 1.4, the homotopy group $\pi_{m}(\mathcal{N})$ endowed with the norm naturally induced by a Sobolev energy satisfies a sufficient condition for compactness of the currents with 
coefficients on an abelian group [17, assumption (H), lemma 7.4 and corollary 7.5] (when $m=1$, this only makes sense when the group $\pi_{1}(\mathcal{N})$ is abelian).

When $m \geq 2$, in analogy with the optimal scaling $\varepsilon^{m}$ when $\varepsilon \rightarrow 0$ of estimates [43], we obtain a similar optimal scaling in $\varepsilon$ (see theorem 5.8 below), with a different strategy of proof than [43].

The proof of theorem 1.3 is performed in a geometric setting where the sphere $\mathbb{S}^{m}$ is considered as the boundary at infinity of the hyperbolic space $\mathbb{H}^{m+1}$ and the manifold $\mathcal{N}$ is embedded isometrically into a Euclidean space $\mathbb{R}^{v}$. The extension of the map $f$ by averaging at each point $x \in \mathbb{H}^{m+1}$ over the sphere at infinity - which is also in fact the hyperharmonic extension - provides a Lipschitz-continuous extension $F: \mathbb{H}^{m+1} \rightarrow \mathbb{R}^{\nu}$. The set on which the values of the map $F$ cannot be retracted to $\mathcal{N}$ is contained in a number of balls whose diameter and number is controlled allowing to construct the families of maps by a classical Ascoli compactness argument for continuous maps.

In view of theorem 1.2, theorem 1.3 describes sharply the homotopy classes that can be encountered under a boundedness assumption on the double integral. However, our proof exhibits a set of maps $\mathscr{F}^{\lambda}$ by a compactness argument and gives thus double exponential bound of the form $\exp \left(C \sinh \left(C^{\prime} \lambda\right)\right)$ on the cardinal of $\mathscr{F}^{\lambda}$. This brings the question whether a better explicit control like the linear estimate (1.5).

In the one-dimensional case, we can estimate the total variation of the maps appearing in the decomposition.

Theorem 1.5 (Estimate on free homotopy decomposition by a truncated fractional energy). If $\varepsilon>0$ is small enough, there is a constant $C>0$ such that every $f \in \mathscr{C}(\mathbb{S}, \mathcal{N})$ has a free decomposition into $f_{1}, \ldots, f_{k} \in$ $\mathscr{C}^{1}\left(\mathbb{S}^{1}, \mathcal{N}\right)$ such that

$$
\sum_{i=1}^{k} \int_{\mathbb{S}^{1}}\left|f_{i}^{\prime}\right| \leq C \underset{\substack{(x, y) \in \mathbb{S}^{1} \times \mathbb{S}^{1} \\ d_{\mathfrak{N}}(f(y), f(x))>\varepsilon}}{ } \frac{1}{|y-x|^{2}} \mathrm{~d} y \mathrm{~d} x
$$

Since $\mathcal{N}$ is a compact manifold, there exists a constant $\eta>0$ such that if $f \in \mathscr{C}^{1}\left(\mathbb{S}^{1}, \mathbb{S}^{1}\right)$ is not homotopic to a constant, then $\int_{\mathbb{S}^{1}}\left|f^{\prime}\right| \geq \eta$ and sets of maps $f \in \mathscr{C}^{1}\left(\mathbb{S}^{1}, \mathbb{S}^{1}\right)$ such that the total variation $\int_{\mathbb{S}^{1}}\left|f^{\prime}\right|$ remains bounded are compact for the uniform convergence and thus intersect finitely many homotopy classes. Hence theorem 1.5 implies theorem 1.3 when $m=1$.

When the homotopy classes can be controlled by the homology, that is, when the Hurewicz homomorphism from $\pi_{m}(\mathcal{N})$ to the rational homology group $H_{m}(\mathcal{N})$ has a finite kernel, we recover a linear control on the number of homotopy classes that satisfy a given bound (see theorem 6.1 below).

When the domain $\mathbb{S}^{m}$ is replaced by a general $m$-dimensional manifold $M$, theorem 1.3 has a natural generalization, in which the corresponding homotopy classes are generated by a finite set of homotopy classes of $\mathscr{C}(\mathcal{N}, \mathcal{N})$ glued together with a finite number of maps taken in finitely many homotopy classes of $\mathscr{C}\left(\mathbb{S}^{m}, \mathcal{N}\right)$ (see section 7 below). As before, there can be in general infinitely many homotopy classes generated in this way by finitely many homotopy classes. The strategy of the proof is similar.

As perspectives of the present work, several open problems are presented in the last section of the present work (see section 8).

\section{Free homotopy decomposition}

\subsection{Free homotopy decomposition and homotopy groups}

The notion of free homotopy decomposition of definition 1.1 plays an important role in the present work. We describe here free homotopy decomposition in terms of homotopy groups.

We define $f \in \mathscr{C}\left(\mathbb{S}^{m}, \mathcal{N}\right)$ and $y \in \pi_{m}(\mathcal{N})$ to be homotopic whenever any representative of the relative homotopy class $y$ is homotopic to the map $f$. Since we have not fixed a base point in the homotopy between 
the representative in $y \in \pi_{m}(\mathcal{N})$ and the map $f$, a given map $f \in \mathscr{C}\left(\mathbb{S}^{m}, \mathcal{N}\right)$ can be homotopic to several distinct elements of $\pi_{m}(\mathcal{N})$.

When $m=1$, the elements of the fundamental group $\pi_{1}(\mathcal{N})$ homotopic to a free homotopy class of maps from the circle $\mathbb{S}^{1}$ to $\mathcal{N}$ form a conjugacy class of the fundamental group $\pi_{1}(\mathcal{N})$ (see for example [27, exercise 1.1.6 and proposition 4A.2]).

Proposition 2.1 (Free decompositions and the fundamental group). Assume that the maps $f, f_{1}, \ldots, f_{k} \in$ $\mathscr{C}\left(\mathbb{S}^{1}, \mathcal{N}\right)$ are respectively homotopic to $y, y_{1}, \ldots, y_{k} \in \pi_{1}(\mathcal{N})$. Then $f$ has a free homotopy decomposition into $f_{1}, \ldots, f_{k}$ if and only if there exist $\beta_{1}, \ldots, \beta_{k} \in \pi_{1}(\mathcal{N})$ such that $y=\beta_{1} y_{1} \beta_{1}^{-1} \cdots \beta_{k} y_{k} \beta_{k}^{-1}$.

In particular, when the fundamental group $\pi_{1}(\mathcal{N})$ is abelian, the homotopy classes of $\mathscr{C}\left(\mathbb{S}^{1}, \mathcal{N}\right)$ correspond to elements in $\pi_{1}(\mathcal{N})$, and the map $f$ has a free homotopy decomposition into $f_{1}, \ldots, f_{k}$ if and only if $y=y_{1} \cdots y_{k}$.

When $m \geq 2$, the elements of $\pi_{m}(\mathcal{N})$ corresponding to a free homotopy class of maps from the circle $\mathcal{N}$ correspond to orbits of the action of the fundamental group $\pi_{1}(\mathcal{N})$ on the homotopy group $\pi_{m}(\mathcal{N})$ (see for example [27, proposition 4A.2]).

Proposition 2.2 (Free decompositions and the homotopy groups). Let $m \geq 2$ and assume that the maps $f, f_{1}, \ldots, f_{k} \in \mathscr{C}\left(\mathbb{S}^{m}, \mathcal{N}\right)$ are respectively homotopic to $y, y_{1}, \ldots, y_{k} \in \pi_{m}(\mathcal{N})$. Then $f$ has a free homotopy decomposition into $f_{1}, \ldots, f_{k}$ if and only if there exists $\beta_{1}, \ldots, \beta_{k} \in \pi_{1}(\mathcal{N})$ such that $y=\beta_{1} \cdot y_{1}+\cdots+\beta_{k} \cdot y_{k}$.

Here $\beta_{i} \cdot y_{i}$ denotes the action of $\beta_{i} \in \pi_{1}(\mathcal{N})$ on $y_{i} \in \pi_{m}(\mathcal{N})$ (see [27, §4.1]). If the action of the fundamental group $\pi_{1}(\mathcal{N})$ on the homotopy group $\pi_{m}(\mathcal{N})$ happens to be trivial, then the map $f$ has a free homotopy decomposition into $f_{1}, \ldots, f_{k}$ if and only if $y=y_{1}+\cdots+y_{k}$.

Proposition 2.3. Assume that $m=1$ and every conjugacy class of $\pi_{1}(\mathcal{N})$ is abelian, or that $m \geq 2$ and every orbit of the action of $\pi_{1}(\mathcal{N})$ on $\pi_{m}(\mathcal{N})$ is finite. If $k \in \mathbb{N}$ and $f_{1}, \ldots, f_{k} \in \mathscr{C}\left(\mathbb{S}^{m}, \mathcal{N}\right)$, then there exists a finite set $\mathscr{G} \subset \mathscr{C}\left(\mathbb{S}^{m}, \mathcal{N}\right)$ such that if $\in \mathscr{C}\left(\mathbb{S}^{m}, \mathcal{N}\right)$ has a free homotopy decomposition into $f_{1}, \ldots, f_{k}$, then $f$ is homotopic to some $g \in \mathscr{G}$.

Proof. When $m=1$, let $y_{1}, \ldots, y_{k} \in \pi_{1}(\mathcal{N})$ be elements of $\pi_{1}(\mathcal{N})$ respectively homotopic to $f_{1}, \ldots, f_{k}$. We consider the set

$$
\Gamma=\left\{\beta_{1} y_{1} \beta_{1}^{-1} \cdots \beta_{k} y_{k} \beta_{k}^{-1} \mid \beta_{1}, \ldots, \beta_{k} \in \pi_{1}(\mathcal{N})\right\} \subseteq \pi_{1}(\mathcal{N}) .
$$

By hypothesis, for every $i \in\{1, \ldots, k\}$, the set $\left\{\beta_{i} y_{i} \beta_{i}^{-1} \mid \beta_{i} \in \pi_{1}(\mathcal{N})\right\}$ is finite and thus the set $\Gamma$ is also finite. We choose $\mathscr{G} \subset \mathscr{C}\left(\mathbb{S}^{1}, \mathcal{N}\right)$ to be a finite set such that each $y \in \Gamma$ is homotopic to some $g \in \mathscr{G}$. By proposition 2.1, any map $f$ that has a free homotopy decomposition into $f_{1}, \ldots, f_{k}$ is homotopic to a map in $\mathscr{G}$.

When $m \geq 2$, the proof is similar and follows from the application of proposition 2.2.

\subsection{Infinitely many homotopy classes sharing the same free homotopy decomposition}

We now show that for some manifolds infinitely many homotopy classes can be decomposed freely into a finite set of maps. This implies in particular that the left-hand side in theorem 1.2 goes through infinitely many homotopy classes.

Proposition 2.4 (Infinitely many homotopy classes sharing a free homotopy decomposition). For every $m \in$ $\mathbb{N}_{\star}$, there exists a compact manifold $\mathcal{N}$ and a map $f \in \mathscr{C}\left(\mathbb{S}^{m}, \mathcal{N}\right)$ such that for every $k \in\{2,3, \ldots\}$, there exists infinitely many homotopy classes in $\mathscr{C}\left(\mathbb{S}^{m}, \mathcal{N}\right)$ having a free homotopy decomposition into $k$ copies of $f$. 
In the one-dimensional case $m=1$, examples can be provided by tori with at least two holes. The next lemma shows that a $g$-hole torus - or equivalently, an orientable surface of genus $g$ - has a fundamental group which is not less complex than a free group on $g$ generators.

Lemma 2.5 (Free group in the fundamental group of $g$-hole tori). If $\mathcal{N}$ is a g-hole torus, then there exists a surjective homomorphism $\tau: \pi_{1}(\mathcal{N}) \rightarrow\left\langle\alpha_{1}, \ldots, \alpha_{g}\right\rangle$.

Here, $\left\langle\alpha_{1}, \ldots, \alpha_{g}\right\rangle$ is the free group on the $g$ generators $\alpha_{1}, \ldots, \alpha_{g}$.

Proof of lemma 2.5. The fundamental group $\pi_{1}(\mathcal{N})$ of the $g$-hole torus $\mathcal{N}$ can be characterized by the group presentation

$$
\pi_{1}(\mathcal{N})=\left\langle a_{1}, b_{1}, \ldots, a_{g}, b_{g} \mid\left[a_{1}, b_{1}\right] \cdots\left[a_{g}, b_{g}\right]=1\right\rangle,
$$

where $\left[a_{i}, b_{i}\right]=a_{i} b_{i} a_{i}^{-1} b_{i}^{-1}\left[27, \S 1.2\right.$, p. 51]. We define the group homomorphism $\hat{\tau}:\left\langle a_{1}, b_{1}, \ldots, a_{g}, b_{g}\right\rangle \rightarrow$ $\left\langle\alpha_{1}, \ldots, \alpha_{g}\right\rangle$ by setting for each $i \in\{1, \ldots, g\}, \hat{\tau}\left(a_{i}\right) \triangleq \alpha_{i}$ and $\hat{\tau}\left(b_{i}\right) \triangleq 1$, and we observe that for each $i \in\{1, \ldots, g\}, \hat{\tau}\left(\left[a_{i}, b_{i}\right]\right)=\left[\hat{\tau}\left(a_{i}\right), \hat{\tau}\left(b_{i}\right)\right]=\left[\alpha_{i}, 1\right]=1$. Hence, we have $\hat{\tau}\left(\left[a_{1}, b_{1}\right] \cdots\left[a_{g}, b_{g}\right]\right)=$ $\hat{\tau}\left(\left[a_{1}, b_{1}\right]\right) \cdots \hat{\tau}\left(\left[a_{g}, b_{g}\right]\right)=1$ and thus $\hat{\tau}$ induces a quotient homomorphism $\tau: \pi_{1}(\mathcal{N}) \rightarrow\left\langle\alpha_{1}, \ldots, \alpha_{g}\right\rangle$. Since $\tau\left(a_{i}\right)=\alpha_{i}$ for each $i \in\{1, \ldots, g\}$, the homomorphism $\tau$ is surjective.

The next lemma will allow us to prove in algebraic terms that maps in $\mathscr{C}\left(\mathbb{S}^{1}, \mathcal{N}\right)$ lie in different homotopy groups.

Lemma 2.6 (Nonconjugacy along a conjugation orbit in a free group). If $k \in\{2,3, \ldots\}$ and if $\ell, j \in \mathbb{N}$, then there exists $h \in\left\langle\alpha_{1}, \ldots, \alpha_{g}\right\rangle$ such that

$$
h^{-1} \alpha_{1} \alpha_{2}^{-\ell} \alpha_{1}^{k-1} \alpha_{2}^{\ell} h=\alpha_{1} \alpha_{2}^{-j} \alpha_{1}^{k-1} \alpha_{2}^{j}
$$

if and only if $\ell=j$.

Proof. If $k=\ell$ the statement holds with $h=1$

Conversely, it can be observed that $\alpha_{1} \alpha_{2}^{-\ell} \alpha_{1}^{k-1} \alpha_{2}^{\ell}$ and $\alpha_{1} \alpha_{2}^{-j} \alpha_{1}^{k-1} \alpha_{2}^{j}$ are cyclically reduced words which can be conjugate in a free group if and only the words are cyclic permutation of each other [32, theorem 1.3]. The statement can also be proved directly. We assume by contradiction that $\ell>k \geq 0$ and that there exists $h \in\left\langle\alpha_{1}, \ldots, \alpha_{g}\right\rangle$ such that the identity holds. Then both corresponding reduced words should have the same length. Since $\ell>j \geq 0$, this means that there should be $(\ell-j)+$ length $(h)$ cancellations between inverses on the left-hand side, and thus at least one cancellation at the beginning and one cancellation at the end of the word on the left-hand side. Since $\ell \neq 0$, the cancellation on the left implies that the first letter of $h$ is $\alpha_{1}$ and the cancellation on the right that the first letter of $h$ is $\alpha_{2}$; this is a contradiction.

Proof of proposition 2.4 when $m=1$. We take the manifold $\mathcal{N}$ to be a $g$-hole torus, with $g \geq 2$. Let $\tau: \pi_{1}(\mathcal{N}) \rightarrow$ $\left\langle\alpha_{1}, \ldots, \alpha_{g}\right\rangle$ be the homomorphism of lemma 2.5 and let $f \in C\left(\mathbb{S}^{1}, \mathcal{N}\right)$ be homotopic to $a_{1} \in \tau^{-1}\left(\left\{\alpha_{1}\right\}\right) \subset$ $\pi_{1}(\mathcal{N})$. We also fix $a_{2} \in \tau^{-1}\left(\left\{\alpha_{2}\right\}\right)$. For every natural number $\ell \in \mathbb{N}$, we choose $f_{\ell} \in \mathscr{C}\left(\mathbb{S}^{1}, \mathcal{N}\right)$ that is homotopic to $a_{1} a_{2}^{-\ell} a_{1}^{k-1} a_{2}^{\ell} \in \pi_{1}(\mathcal{N})$. By proposition 2.1, the map $f_{\ell}$ has a free homotopy decomposition into $k$ copies of the map $f$. If for some $\ell, j \in \mathbb{N}$, the maps $f_{\ell}$ and $f_{j}$ are homotopic, then $a_{1} a_{2}^{-\ell} a_{1}^{k-1} a_{2}^{\ell}$ and $a_{1} a_{2}^{-j} a_{1}^{k-1} a_{2}^{j}$ are conjugate in $\pi_{1}(\mathcal{N})$ and thus, since $\tau$ is a homomorphism, we deduce that $\alpha_{1} \alpha_{2}^{-\ell} \alpha_{1}^{k-1} \alpha_{2}^{\ell}$ and $\alpha_{1} \alpha_{2}^{-j} \alpha_{1}^{k-1} \alpha_{2}^{j}$ are conjugate in the free group $\left\langle\alpha_{1}, \ldots, \alpha_{g}\right\rangle$. By lemma 2.6, this implies that $\ell=j$, and thus the maps $f_{\ell}$ and $f_{j}$ are homotopic if and only if $\ell=j$.

For $m \geq 2$, we rely on the following construction of manifolds:

Lemma 2.7 (Manifold with nontrivial action by the fundamental group). For every $m \geq 2$, there exists $a(2 m+$ 1)-dimensional compact Riemannian manifold $\mathcal{N}$ isometrically embedded into $\mathbb{R}^{2 m+2}$ such that $\pi_{1}(\mathcal{N}) \simeq \mathbb{Z}$, $\pi_{m}(\mathcal{N}) \simeq \mathbb{Z}^{\mathbb{Z}}$ and $\pi_{1}(\mathcal{N})$ acts on $\pi_{m}(\mathcal{N})$ as the translation operator. 
Proof. If $X \triangleq \mathbb{S}^{1} \vee \mathbb{S}^{m}$ is the CW complex obtained by the bouquet construction applied between the circle $\mathbb{S}^{1}$ and the sphere $\mathbb{S}^{m}$, then $\pi_{1}(X) \simeq \mathbb{Z}, \pi_{m}(X) \simeq \mathbb{Z}^{\mathbb{Z}}$ and $\pi_{1}(X)$ acts on $\pi_{m}(X)$ as the translation operator (see for example [27, example 4.27]).

We embed the CW complex $X$ in the Euclidean space $\mathbb{R}^{2 m+2}$ and we consider a neighborhood $U$ of $X$ in $\mathbb{R}^{2 m+2}$ that has a smooth boundary and such that $X$ is a retraction of $U$ and $\partial U$ is a retraction of $U \backslash X$. We define $\mathcal{N} \triangleq \partial U$.

We then observe that any Lipschitz-continuous homotopy $h: \mathbb{S}^{k} \times[0,1] \rightarrow U$ has a $(k+1)$-dimensional image. Since the set $U \subset \mathbb{R}^{2 m+2}$ is open, if $k \leq m$, the homotopy $h$ can be perturbed in such a way of not intersecting the $m$-dimensional set $X$. This implies that $\pi_{1}(\mathcal{N}) \simeq \pi_{1}(U \backslash X) \simeq \pi_{1}(U) \simeq \pi_{1}(X) \simeq \mathbb{Z}$ and $\pi_{m}(\mathcal{N}) \simeq \pi_{m}(U \backslash X) \simeq \pi_{m}(U) \simeq \pi_{m}(X) \simeq \mathbb{Z}^{\mathbb{Z}}$, with isomorphisms between the actions of $\pi_{1}(\mathcal{N})$ on $\pi_{m}(\mathcal{N})$ and of $\pi_{1}(X)$ on $\pi_{m}(X)$.

The manifold $\mathcal{N}$ constructed in the proof of lemma 2.7 can be described as the result of gluing $\mathbb{S}^{1} \times \mathbb{S}^{2 m}$ to $\mathbb{S}^{m} \times \mathbb{S}^{m+1}$ along a trivial sphere $\mathbb{S}^{2 m}$.

Remark 2.8. When $m=2$, the construction of the proof of lemma 2.7 yields a 3-dimensional compact Riemannian manifold $\mathcal{N}$ embedded into $\mathbb{R}^{4}$ such that $\pi_{1}(\mathcal{N})$ is a free group on two generators.

Proof of proposition 2.4 when $m \geq 2$. Let $\mathcal{N}$ be the manifold given by lemma 2.7. We fix a map $f \in \mathscr{C}\left(\mathbb{S}^{m}, \mathcal{N}\right)$ that is not homotopic to a constant and we choose $a_{0} \in \pi_{m}(\mathcal{N})$ homotopic to $f$. For each $k \in \mathbb{Z}$, let $a_{k}$ be the result of the action of $k \in \mathbb{Z} \simeq \pi_{1}(\mathcal{N})$ on $a_{0} \in \pi_{m}(\mathcal{N})$. By proposition 2.3, the homotopy classes that have a free homotopy decomposition into $k$ copies of the map $f$ correspond to sets of the form $\left\{a_{i_{1}+\ell}+\cdots a_{i_{k}+\ell} \mid \ell \in \mathbb{Z}\right\}$, with $i_{1}, \ldots, i_{k} \in \mathbb{Z}$. If $k \geq 2$, there are infinitely many such sets.

\section{Upper bound on Sobolev energies by free homotopy decomposition}

Theorem 1.2 will be obtained by induction from the corresponding result with $k=2$ :

Proposition 3.1 (Estimate of Sobolev energy by free homotopy decomposition into two maps). Let $m \in \mathbb{N}_{*}$, $\mathcal{N}$ be a connected Riemannian manifold $s \in(0,1]$ and $p \in[m,+\infty)$. If $p=m / s>1$ and if $f \in \mathscr{C}\left(\mathbb{S}^{m}, \mathcal{N}\right)$ has a free homotopy decomposition into $f_{+}, f_{-} \in\left(\mathscr{C} \cap W^{s, p}\right)\left(\mathbb{S}^{m}, \mathcal{N}\right)$, then

$$
\inf \left\{\mathcal{E}^{s, p}(g) \mid g \in\left(\mathscr{C} \cap W^{s, p}\right)\left(\mathbb{S}^{m}, \mathcal{N}\right) \text { is homotopic to } f\right\} \leq \mathcal{E}^{s, p}\left(f_{+}\right)+\mathcal{E}^{s, p}\left(f_{-}\right) .
$$

Computations will be facilitated by parametrizing the sphere $\mathbb{S}^{m}$ through its Mercator projection on the cylin$\operatorname{der} \mathbb{S}^{m-1} \times \mathbb{R}$. When $m=2$, this corresponds to the projection used by Mercator on the cylinder to cartography the earth. The Mercator projection is a conformal transformation, and preserves thus the critical Sobolev energy.

Lemma 3.2 (Conformal derivative integrals under Mercator cylindrical projection). For every $m \in \mathbb{N}_{\star}$ and for every $f \in \mathbb{S}^{m} \rightarrow \mathcal{N}$, we have $f \in W^{1, m}\left(\mathbb{S}^{m}, \mathcal{N}\right)$ if and only if $f \circ Y \in W^{1, m}\left(\mathbb{S}^{m-1} \times \mathbb{R}, \mathcal{N}\right)$, where the map $Y: \mathbb{S}^{m-1} \times \mathbb{R} \rightarrow \mathbb{S}^{m}$ is defined for each $(z, s) \in \mathbb{S}^{m-1} \times \mathbb{R}$ by $Y(z, s) \triangleq(z$ sech $s$, tanh $s)$. Moreover,

$$
\int_{\mathbb{S}^{m}}|D f|^{m}=\int_{\mathbb{R} \times \mathbb{S}^{m-1}}|D(f \circ Y)|^{m} .
$$

Proof. We compute, if $(z, s) \in \mathbb{S}^{m-1} \times \mathbb{R}$, if $(u, r) \in \mathbb{R}^{m} \times \mathbb{R}$ and if $u \cdot z=0$,

$$
\begin{aligned}
|D Y(z, s)[(r, u)]|^{2} & =|z r \operatorname{sech} s \tanh s-u \operatorname{sech} s|^{2}+\left|r(\operatorname{sech} s)^{2}\right|^{2} \\
& =r^{2}(\operatorname{sech} s)^{2}\left((\operatorname{sech} s)^{2}+(\tanh s)^{2}\right)+|u|^{2} \operatorname{sech} s^{2} \\
& =(\operatorname{sech} s)^{2}\left(|r|^{2}+|u|^{2}\right) ;
\end{aligned}
$$


since $|z|=1$. It thus follows that the mapping $Y$ is conformal and the identity holds.

The fractional counterpart of lemma 3.2 is an identity between the fractional integral on the sphere and a fractional integral with exponentially decaying potential in the longitudinal direction of the cylinder.

Lemma 3.3 (Conformal fractional integrals under Mercator cylindrical projection). For every $m \in \mathbb{N}_{\star}$, for every $p \in(0,+\infty)$ and for every $f: \mathbb{S}^{m} \rightarrow \mathcal{N}$,

$$
\int_{\mathbb{S}^{m}} \int_{\mathbb{S}^{m}} \frac{d_{\mathcal{N}}(f(y), f(x))^{p}}{|y-x|^{2 m}} \mathrm{~d} y \mathrm{~d} x=\int_{\mathbb{S}^{m-1}} \int_{\mathbb{R}} \int_{\mathbb{S}^{m-1}} \int_{\mathbb{R}} \frac{d_{\mathcal{N}}(f(w \operatorname{sech} t, \tanh t), f(z \operatorname{sech} s, \tanh s))^{p}}{\left(\left(2 \sinh \frac{t-s}{2}\right)^{2}+|w-z|^{2}\right)^{m}} \mathrm{~d} t \mathrm{~d} w \mathrm{~d} s \mathrm{~d} z .
$$

Proof. We define the Mercator projection $Y: \mathbb{S}^{m-1} \times \mathbb{R} \rightarrow \mathbb{S}^{m}$ as in the statement of lemma 3.2 and we observe that (3.1) holds and thus for every $(z, s) \in \mathbb{S}^{m-1} \times \mathbb{R}$, we have Jac $Y(z, s)=\operatorname{sech} s$. Moreover, if $(z, s),(w, t) \in$ $\mathbb{S}^{m-1} \times \mathbb{R}$, since $|z|=|w|=1$, we have

$$
\begin{aligned}
|Y(t, w)-Y(s, z)|^{2} & =|w \operatorname{sech} t-z \operatorname{sech} s|^{2}+|\tanh t-\tanh s|^{2} \\
& =\operatorname{sech} t \operatorname{sech} s|w-z|^{2}+|\operatorname{sech} t-\operatorname{sech} s|^{2}+|\tanh t-\tanh s|^{2} \\
& =\operatorname{sech} t \operatorname{sech} s\left(|w-z|^{2}+2(\cosh t \cosh s-\sinh t \sinh s-1)\right) \\
& =\operatorname{sech} t \operatorname{sech} s\left(|w-z|^{2}+2(\cosh (t-s)-1)\right) \\
& =\operatorname{sech} t \operatorname{sech} s\left(\left(2 \sinh \frac{t-s}{2}\right)^{2}+|w-z|^{2}\right) .
\end{aligned}
$$

The identity follows then by a change of variable $x=Y(z, s)$ and $y=Y(w, t)$.

The proof of proposition 3.1 also relies on a construction of maps that are constant on some set.

Lemma 3.4 (Approximation of the identity by maps degenerate at a point). For every $b \in \mathcal{N}$ and every $\varepsilon>0$, there exists a map $\Theta \in \mathscr{C}^{1}(\mathcal{N}, \mathcal{N})$ which is homotopic to the identity and such that $\Theta=b$ in a neighborhood of $b$ and for every $y, z \in \mathcal{N}, d(\Theta(z), \Theta(y)) \leq(1+\varepsilon) d(z, y)$.

Proof. We fix a function $\eta \in \mathscr{C}^{\infty}(\mathbb{R},[0,+\infty))$ such that $\eta=0$ on $(-\infty,-2]$ and $\eta=1$ on $[-1,+\infty)$. We first define the function $\Xi_{\lambda}: \mathbb{R}^{m} \rightarrow \mathbb{R}^{m}$ for $\lambda \in(0,+\infty)$ and for $u \in \mathbb{R}^{m}$ by $\Xi_{\lambda}(u) \triangleq \eta(\lambda \ln |u|) u$, and we observe that for every $u, v \in \mathbb{R}^{m},\left|D \Xi_{\lambda}(u)[v]\right| \leq\left(1+C_{1} \lambda\right)|v|$. We define now for each $y \in \mathcal{N}$,

$$
\Theta_{\lambda}(y) \triangleq \begin{cases}\exp _{b}\left(\Xi_{\lambda}\left(\exp _{b}^{-1}(y)\right)\right) & \text { if } d(y, b) \leq \operatorname{inj}_{\mathcal{N}}(b) \\ y & \text { otherwise. }\end{cases}
$$

where $\exp _{b}$ is the Riemannian exponential map on $\mathcal{N}$ at $b$ and $\operatorname{inj}_{\mathcal{N}}(b)$ is the injectivity radius of the Riemannian manifold $\mathcal{N}$ at the point $b$. We obtain the conclusion by taking $\lambda>0$ small enough.

Proof of proposition 3.1. We choose a coordinate system so that $a=(0, \ldots, 0,1) \in \mathbb{S}^{m} \subset \mathbb{R}^{m+1}$. By lemma 3.4, for every $\varepsilon>0$, there exists maps $\Theta^{ \pm}: \mathcal{N} \rightarrow \mathcal{N}$ that are constant in a neighborhood of the point $f_{ \pm}(\mp a)$. It follows then that $g_{+} \triangleq \Theta^{+} \circ f_{+}$is constant in a neighborhood of $-a$ and $g_{-} \triangleq \Theta^{-} \circ f_{-}$is constant in a neighborhood of $a$ and

$$
\varepsilon^{s, p}\left(g_{ \pm}\right) \leq(1+\varepsilon)^{p} \mathcal{E}^{s, p}\left(f_{ \pm}\right) .
$$

Up to a homotopy, we can consider that the map $f$ is constant in a neighborhood of the equator $\partial B_{\pi / 2}(a)=$ $\partial B_{\pi / 2}(-a)$, that $\left.f\right|_{\bar{B}_{\pi / 2}(a)}$ is homotopic to $f_{+}$on $\mathbb{S}^{m} \simeq \bar{B}_{\pi / 2}(a) / \partial B_{\pi / 2}(a)$ and that $f_{\bar{B}_{\pi / 2}(-a)}$ is homotopic to $f_{-}$on $\mathbb{S}^{m} \simeq \bar{B}_{\pi / 2}(-a) / \partial B_{\pi / 2}(-a)$.

We consider the cylinder $K=\partial\left(\mathbb{B}^{m} \times[-1,1]\right)=\mathbb{B}^{m} \times\{-1,1\} \cup \mathbb{S}^{m-1} \times[-1,1]$ and a map $\Phi: K \rightarrow \mathbb{S}^{m}$ such that $\left.\Phi\right|_{\mathbb{B}^{m} \times\{ \pm 1\}}$ is a homeomorphism with $B_{\pi / 2}( \pm a)$ and $\Phi(x, s)=x$ for every $(x, s) \in \mathbb{S}^{m-1} \times[-1,1]$. If we define the mapping $\Psi: K \rightarrow \mathbb{S}^{m}$ by $\Psi(x, s) \triangleq(x, s) /|(x, s)|$, we observe that $\Psi$ is a homeomorphism and that the maps $\Psi$ and $\Phi$ are homotopic.

Since $f$ is homotopic to $g_{ \pm}$on $\mathbb{S}^{m} \simeq \bar{B}_{\pi / 2}( \pm a) / \partial B_{\pi / 2}( \pm a)$, there exists a homotopy $H \in \mathscr{C}\left(\left(\mathbb{B}^{m} \times\{-1,1\}\right) \times\right.$ $[0,1], \mathcal{N})$ such that $H(\cdot, 0)=f \circ \Phi$ on $\mathbb{B}^{m} \times\{-1,1\}, H(\cdot, \pm 1,1)=g_{ \pm} \circ \Phi$ on $\mathbb{B}^{m}$ and $H(\cdot, t)$ is constant for every 
$t \in[0,1]$ on both sets $\partial \mathbb{B}^{m} \times\{-1\}$ and $\partial \mathbb{B}^{m} \times\{1\}$ (with possibly constant values differing on one set from the other). By the homotopy extension property (see for example [27, Proposition 0.16]), there exists a homotopy $\Gamma \in \mathscr{C}([-1,1] \times[0,1], \mathcal{N})$ such that $\Gamma(s, 0)=\left.f\right|_{\partial B_{\pi / 2}(a)}$ and for every $t \in[0,1], \Gamma( \pm 1, t)=H(\cdot, \pm 1, t)$ on $\partial \mathbb{B}^{m}$. We define the map $y \in \mathscr{C}([0,1], \mathcal{N})$ for each $s \in[0,1]$ by $y(s) \triangleq \Gamma(s, 1)$. By a regularization argument, we can assume that $y=\left.\bar{y}\right|_{[-1,1]}$ for some $\bar{y} \in \mathscr{C}^{1}(\mathbb{R}, \mathcal{N})$ such that $\bar{y}=g_{-}(a)$ on $(-\infty,-1]$ and $\bar{y}=g_{+}(a)$ on $[1,+\infty)$. We observe that $f \circ \Phi$ is homotopic to the map $h: K \rightarrow \mathcal{N}$ defined for $(x, s) \in K$ by

$$
h(x, s) \triangleq \begin{cases}g_{-}(\Phi(x, s)) & \text { if } s=-1, \\ y(s) & \text { if }-1<s<1, \\ g_{+}(\Phi(x, s)) & \text { if } s=1 .\end{cases}
$$

It follows then that $h \circ \Psi^{-1}$ is homotopic to $f$ on $\mathbb{S}^{m}$.

We now consider the maps $\tilde{g}_{ \pm}: \mathbb{S}^{m-1} \times \mathbb{R} \rightarrow \mathcal{N}$ defined for $(z, s) \in \mathbb{S}^{m-1} \times \mathbb{R}$ by

$$
\tilde{g}_{ \pm}(z, s) \triangleq g_{ \pm}(z \operatorname{sech} s, \tanh s) .
$$

We observe that there exists $s_{+} \in \mathbb{R}$ such that if $s \leq-s_{+}$and $z \in \mathbb{S}^{m-1}$, then $\tilde{g}_{+}(z, s)=g_{+}\left(a_{-}\right)$. Similarly, there exists $s_{-} \in \mathbb{R}$ such that if $s \geq s_{-}$and $z \in \mathbb{S}^{m-1}$, then $\tilde{g}_{-}(z, s)=g_{-}\left(a_{+}\right)$. We construct now for each $\lambda \in(0,+\infty)$, the map $\tilde{g}_{\lambda}: \mathbb{S}^{m-1} \times \mathbb{R} \rightarrow \mathcal{N}$ by setting for each $(z, s) \in \mathbb{S}^{m-1} \times \mathbb{R}$,

$$
\tilde{g}_{\lambda}(z, s) \triangleq \begin{cases}\tilde{g}_{-}\left(z, s+2 \lambda+s_{-}\right) & \text {if } s \in(-\infty,-2 \lambda], \\ y(s / \lambda) & \text { if } s \in[-2 \lambda, 2 \lambda], \\ \tilde{g}_{+}\left(z, s-2 \lambda-s_{+}\right) & \text {if } s \in[2 \lambda,+\infty) .\end{cases}
$$

We define now for every $\lambda \in(0,+\infty)$ the map $g_{\lambda}: \mathbb{S}^{m} \rightarrow \mathcal{N}$ for each $(y, t) \in \mathbb{S}^{m} \subset \mathbb{R}^{m} \times \mathbb{R}$,

$$
g_{\lambda}(y, t) \triangleq \tilde{g}_{\lambda}\left(y /|y|, \tanh ^{-1}(t)\right) .
$$

By construction, the map $g_{\lambda}$ is homotopic to $h \circ \Psi^{-1}$ on $\mathbb{S}^{m}$, which in turn is homotopic to the map $f$ on $\mathbb{S}^{m}$. It remains to estimate its Sobolev energy $\mathcal{E}^{s, p}\left(g_{\lambda}\right)$.

If $s=1$, we have by lemma 3.2,

$$
\begin{aligned}
\mathcal{E}^{1, m}\left(g_{\lambda}\right) & =\int_{\mathbb{S}^{m}}\left|D g_{\lambda}\right|^{m}=\int_{\mathbb{S}^{m-1} \times \mathbb{R}}\left|D \tilde{g}_{\lambda}\right|^{m} \\
& =\int_{\mathbb{S}^{m-1} \times\left(-\infty, s_{-}\right]}\left|D \tilde{\boldsymbol{g}}_{-}\right|^{m}+\left|\mathbb{S}^{m-1}\right| \lambda^{m-1} \int_{-1}^{1}\left|y^{\prime}\right|^{m}+\int_{\mathbb{S}^{m-1} \times\left[-s_{+},+\infty\right)}\left|D \tilde{g}_{+}\right|^{m} \\
& \leq \mathcal{E}^{1, m}\left(g_{+}\right)+\mathcal{E}^{1, m}\left(g_{-}\right)+C_{1} \lambda^{m-1} .
\end{aligned}
$$

The conclusion then follows by letting $\lambda \rightarrow 0$ and $\varepsilon \rightarrow 0$.

If $0<s<1$, we have by lemma 3.3,

$$
\int_{\mathbb{S}^{m}} \int_{\mathbb{S}^{m}} \frac{d_{\mathcal{N}}\left(g_{ \pm}(y), g_{ \pm}(x)\right)^{p}}{|y-x|^{2 m}} \mathrm{~d} y \mathrm{~d} x=\int_{\mathbb{S}^{m-1}} \int_{\mathbb{R}} \int_{\mathbb{S}^{m-1}} \int_{\mathbb{R}} \frac{d_{\mathcal{N}}\left(\tilde{g}_{ \pm}(w, t), \tilde{g}_{ \pm}(z, s)\right)^{p}}{\left(\left(2 \sinh \frac{t-s}{2}\right)^{2}+|w-z|^{2}\right)^{m}} \mathrm{~d} t \mathrm{~d} w \mathrm{~d} s \mathrm{~d} z .
$$

and for every $\lambda>0$,

$$
\int_{\mathbb{S}^{m}} \int_{\mathbb{S}^{m}} \frac{d_{\mathcal{N}}\left(g_{\lambda}(y), g_{\lambda}(x)\right)^{p}}{|y-x|^{2 m}} \mathrm{~d} y \mathrm{~d} x=\int_{\mathbb{S}^{m-1}} \int_{\mathbb{R}} \int_{\mathbb{S}^{m-1}} \int_{\mathbb{R}} \frac{d_{\mathcal{N}}\left(\tilde{g}_{\lambda}(w, t), \tilde{g}_{\lambda}(z, s)\right)^{p}}{\left(\left(2 \sinh \frac{t-s}{2}\right)^{2}+|w-z|^{2}\right)^{m}} \mathrm{~d} t \mathrm{~d} w \mathrm{~d} s \mathrm{~d} z .
$$

We first estimate the tails in (3.4)

$$
\int_{\mathbb{S}^{m-1}} \int_{-\infty}^{-\lambda} \int_{\mathbb{S}^{m-1}} \int_{-\infty}^{-\lambda} \frac{d_{\mathcal{N}}\left(\tilde{g}_{\lambda}(w, t), \tilde{g}_{\lambda}(z, s)\right)^{p}}{\left(\left(2 \sinh \frac{t-s}{2}\right)^{2}+|w-z|^{2}\right)^{m}} \mathrm{~d} t \mathrm{~d} w \mathrm{~d} s \mathrm{~d} z
$$




$$
\leq \int_{\mathbb{S}^{m-1}} \int_{\mathbb{R}} \int_{\mathbb{S}^{m-1}} \int_{\mathbb{R}} \frac{d_{\mathcal{N}}\left(\tilde{g}_{-}(w, t), \tilde{g}_{-}(z, s)\right)^{p}}{\left(\left(2 \sinh \frac{t-s}{2}\right)^{2}+|w-z|^{2}\right)^{m}} \mathrm{~d} t \mathrm{~d} w \mathrm{~d} s \mathrm{~d} z
$$

and

$$
\begin{aligned}
& \int_{\mathbb{S}^{m-1}} \int_{\lambda}^{+\infty} \int_{\mathbb{S}^{m-1}} \int_{\lambda}^{+\infty} \frac{d_{\mathcal{N}}\left(\tilde{g}_{\lambda}(w, t), \tilde{g}_{\lambda}(z, s)\right)^{p}}{\left(\left(2 \sinh \frac{t-s}{2}\right)^{2}+|w-z|^{2}\right)^{m}} \mathrm{~d} s \mathrm{~d} w \mathrm{~d} t \mathrm{~d} z \\
& \quad \leq \int_{\mathbb{S}^{m-1}} \int_{\mathbb{R}} \int_{\mathbb{S}^{m-1}} \int_{\mathbb{R}} \frac{d_{\mathcal{N}}\left(\tilde{g}_{+}(w, t), \tilde{g}_{+}(z, s)\right)^{p}}{\left(\left(2 \sinh \frac{t-s}{2}\right)^{2}+|w-z|^{2}\right)^{m}} \mathrm{~d} t \mathrm{~d} w \mathrm{~d} s \mathrm{~d} z
\end{aligned}
$$

Next, if $m \geq 2$, we apply a change of variable through a stereographic projection on $\mathbb{S}^{m-1}$ : for $v \in z^{\perp} \simeq$ $\mathbb{R}^{m-1}$, we set $w=\Sigma_{z}(v) \triangleq\left(z\left(1-|v|^{2}\right)+2 v\right) /\left(1+|v|^{2}\right)$, so that, since $|w|=1$, we have $\left|w-\Sigma_{z}(v)\right|^{2}=4|v|^{2} /\left(1+|v|^{2}\right)$, and, for every $k \in z^{\perp},\left|D \Sigma_{z}(v)[k]\right|=2|k| /\left(1+|v|^{2}\right)$, so that Jac $\Sigma_{z}=2^{m-1} /\left(1+|v|^{2}\right)^{m-1}$ and therefore, for every $s, t \in[-2 \lambda, 2 \lambda]$,

$$
\begin{aligned}
\int_{\mathbb{S}^{m-1}} \int_{\mathbb{S}^{m-1}} & \frac{d_{\mathcal{N}}\left(\tilde{g}_{\lambda}(w, t), \tilde{g}_{\lambda}(z, s)\right)^{p}}{\left(\left(\sinh \frac{t-s}{2}\right)^{2}+|w-z|^{2}\right)^{m}} \mathrm{~d} w \mathrm{~d} z \\
& =C_{2} d_{\mathcal{N}}(y(t / \lambda), y(s / \lambda))^{p} \int_{\mathbb{R}^{m-1}} \frac{\left(1+|v|^{2}\right)}{\left(\left(\sinh \frac{t-s}{2}\right)^{2}\left(1+|v|^{2}\right)+|v|^{2}\right)^{m}} \mathrm{~d} v \\
& =C_{3} d_{\mathcal{N}}(y(t / \lambda), y(s / \lambda))^{p} \int_{0}^{+\infty} \frac{\left(1+r^{2}\right) r^{m-2}}{\left(\left(2 \sinh \frac{t-s}{2}\right)^{2}\left(1+r^{2}\right)+r^{2}\right)^{m}} \mathrm{~d} r \\
& \leq \frac{C_{3}}{\left(2 \sinh \frac{t-s}{2}\right)^{2}} d_{\mathcal{N}}(y(t / \lambda), y(s / \lambda))^{p} \int_{0}^{+\infty} \frac{1}{\left(\left(2 \sinh \frac{t-s}{2}\right)^{2}\left(1+r^{2}\right)+r^{2}\right)^{\frac{m}{2}}} \mathrm{~d} r \\
& \leq \frac{C_{4}}{\left(\sinh \frac{t-s}{2}\right)^{2}} d_{\mathcal{N}}(y(t / \lambda), y(s / \lambda))^{p} \int_{0}^{+\infty} \frac{1}{\left(\left|\sinh \frac{t-s}{2}\right|+r\right)^{m}} \mathrm{~d} r \\
& =\frac{C_{5} d_{\mathcal{N}}(y(t / \lambda), y(s / \lambda))^{p}}{\left|\sinh \frac{t-s}{2}\right|^{m+1}} .
\end{aligned}
$$

The same estimate still holds when $m=1$. We have thus

$$
\begin{aligned}
\int_{\mathbb{S}^{m-1}} \int_{-2 \lambda}^{2 \lambda} \int_{\mathbb{S}^{m-1}} \int_{-2 \lambda}^{2 \lambda} \frac{d_{\mathcal{N}}\left(\tilde{g}_{\lambda}(w, t), \tilde{g}_{\lambda}(z, s)\right)^{p}}{\left(\left(2 \sinh \frac{t-s}{2}\right)^{2}+|w-z|^{2}\right)^{m}} \mathrm{~d} t \mathrm{~d} w \mathrm{~d} s \mathrm{~d} z & \leq C_{5} \int_{-2 \lambda}^{2 \lambda} \int_{-2 \lambda}^{2 \lambda} \frac{d_{\mathcal{N}}(y(t / \lambda), y(s / \lambda))^{p}}{\left|\sinh \frac{t-s}{2}\right|^{m+1}} \mathrm{~d} t \mathrm{~d} s \\
& \leq C_{6} \frac{1}{\lambda^{p}} \int_{-2 \lambda}^{2 \lambda} \int_{-2 \lambda}^{2 \lambda} \frac{|t-s|^{p}}{\left|\sinh \frac{t-s}{2}\right|^{m+1}} \mathrm{~d} t \mathrm{~d} s \leq \frac{C_{7}}{\lambda^{p-1}} .
\end{aligned}
$$

Finally, we observe that if $\left.(s, t) \in \mathbb{R}^{2} \backslash(]-\infty,-\lambda\right]^{2} \cup[-2 \lambda, 2 \lambda]^{2} \cup[\lambda,+\infty)^{2}$ and $s \leq t$, then $s \leq t-\lambda, s \leq \lambda$ and $t \geq-\lambda$. We have then, under the changes of variables $\sigma=t-s$ and $\tau=t+\lambda$,

$$
\begin{aligned}
& \iint_{\substack{(s, t) \in \mathbb{R}^{2} \\
s \leq t-\lambda \\
s \leq \lambda \\
t \geq-\lambda}} \int_{\mathbb{S}^{m-1}} \int_{\mathbb{S}^{m-1}} \frac{d_{\mathcal{N}}\left(\tilde{g}_{\lambda}(w, t), \tilde{g}_{\lambda}(z, s)\right)^{p}}{\left(\left(2 \sinh \frac{t-s}{2}\right)^{2}+|w-z|^{2}\right)^{m}} \mathrm{~d} w \mathrm{~d} z \mathrm{~d} s \mathrm{~d} t \\
& \quad C_{8} \int_{-\lambda}^{+\infty} \int_{-\infty}^{\min (t-\lambda, \lambda)} \frac{1}{\left|\sinh \frac{t-s}{2}\right|^{2 m}} \mathrm{~d} s \mathrm{~d} t=C_{8} \int_{0}^{+\infty} \int_{\max (\lambda, \tau-2 \lambda)}^{+\infty} \frac{1}{\left|\sinh \frac{\sigma}{2}\right|^{2 m}} \mathrm{~d} \sigma \mathrm{d} \tau \\
& \quad \leq C_{9} \int_{0}^{+\infty} \int_{\frac{\lambda+\tau}{4}}^{+\infty} \frac{1}{\left|\sinh \frac{\sigma}{2}\right|^{2 m}} \mathrm{~d} \sigma \mathrm{d} \tau \leq C_{10} \int_{0}^{+\infty} e^{-\frac{m}{4}(\lambda+\tau)} \mathrm{d} \tau=\frac{4 C_{10} e^{-\frac{m \lambda}{4}}}{m},
\end{aligned}
$$

since $\frac{\lambda+\tau}{4} \leq \frac{\tau-2 \lambda}{4}+\frac{3 \lambda}{4} \leq \max (\tau-2 \lambda, \lambda)$. By combining the identities (3.4) and (3.3) together with the estimates (3.5), (3.6), (3.7) and (3.8), we obtain

$$
\int_{\mathbb{S}^{m}} \int_{\mathbb{S}^{m}} \frac{d_{\mathcal{N}}\left(g_{\lambda}(y), g_{\lambda}(x)\right)^{p}}{|y-x|^{2 m}} \mathrm{~d} y \mathrm{~d} x \leq \int_{\mathbb{S}^{m}} \int_{\mathbb{S}^{m}} \frac{d_{\mathcal{N}}\left(g_{+}(y), g_{+}(x)\right)^{p}}{|y-x|^{2 m}} \mathrm{~d} y \mathrm{~d} x+\int_{\mathbb{S}^{m}} \int_{\mathbb{S}^{m}} \frac{d_{\mathcal{N}}\left(g_{-}(y), g_{-}(x)\right)^{p}}{|y-x|^{2 m}} \mathrm{~d} y \mathrm{~d} x+\frac{C_{11}}{\lambda^{p-1}},
$$

and we reach thus the conclusion, by taking $\lambda>0$ and $\varepsilon>0$ arbitrarily small. 


\section{Estimates of free homotopy decomposition on the sphere}

\subsection{Extension}

In order to prove theorem 1.3, we first extend the map $f$ on the sphere $\mathbb{S}^{m}$ to a map $F$ on the ball $\mathbb{B}^{m+1}$ taking its value into the ambient space, by relying on the next proposition which provides a suitably controlled extension. When we endow the ball $\mathbb{B}^{m+1}$ with the Poincare metric of the hyperbolic space $\mathbb{H}^{m+1}$, that is, if we consider the metric defined as quadratic form for $z \in \mathbb{B}^{m+1}$ and $v \in \mathbb{R}^{m+1}$ by

$$
g_{z}(v) \triangleq \frac{4|v|^{2}}{\left(1-|z|^{2}\right)^{2}},
$$

we obtain uniform estimates on the measure of the set on which the function $F$ is far from the set of values on the boundary $f\left(\mathbb{S}^{m}\right)$.

Proposition 4.1 (Extension to the hyperbolic space). Let $m \in \mathbb{N}_{\star}$. There exists a constant $C>0$ such that for every $v \in \mathbb{N}_{\star}$ and every function $f \in \mathscr{C}^{\infty}\left(\mathbb{S}^{m}, \mathbb{R}^{v}\right)$, there exists a function $F \in \mathscr{C}^{\infty}\left(\overline{\mathbb{B}}^{m+1}, \mathbb{R}^{v}\right) \cap \mathscr{C}^{\infty}\left(\mathbb{B}^{m+1}, \mathbb{R}^{v}\right)$ such that

(i) $\left.F\right|_{\partial \mathbb{B}^{m+1}}=f$,

(ii) for every point $x \in \mathbb{H}^{m+1} \simeq \mathbb{B}^{m+1}$,

$$
|D F(x)|_{\mathbb{H}^{m+1}} \leq \underset{\mathbb{S}^{m}}{\operatorname{mos} f},
$$

(iii) if $\delta>\varepsilon$, then

$$
\mu_{\mathbb{H}^{m+1}}\left(\left\{x \in \mathbb{H}^{m+1} \mid \operatorname{dist}\left(F(x), f\left(\mathbb{S}^{m}\right)\right) \geq \delta\right\}\right) \leq \frac{C}{\delta-\varepsilon} \iint_{\mathbb{S}^{m} \times \mathbb{S}^{m}} \frac{(|f(y)-f(x)|-\varepsilon)_{+}}{|y-x|^{2 m}} \mathrm{~d} y \mathrm{~d} x .
$$

In this statement, the oscillation of the function $f$ is defined as

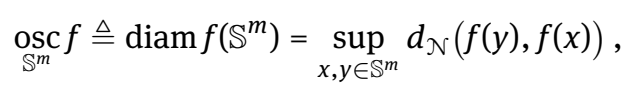

In Euclidean terms, the estimates of proposition 4.1 read in view of the definition of the Poincare metric (4.1) as follows: for every $z \in \mathbb{B}^{m+1}$,

$$
|D F(z)| \leq \frac{2 m \operatorname{osc}_{\mathbb{S}^{m}} f}{1-|z|^{2}}
$$

and for every $\delta>\varepsilon$,

$$
\int_{\substack{x \in \mathbb{H}^{m+1} \\ \operatorname{dist}\left(F(x), f\left(\mathbb{S}^{m}\right)\right) \geq \delta}} \frac{2^{m+1}}{\left(1-|x|^{2}\right)^{m+1}} \mathrm{~d} x \leq \frac{C}{\varepsilon-\delta} \iint_{\mathbb{S}^{m} \times \mathbb{S}^{m}} \frac{(|f(y)-f(x)|-\varepsilon)_{+}}{|y-x|^{2 m}} \mathrm{~d} y \mathrm{~d} x .
$$

When the function $f$ is bounded, the latter inequality (4.2) is a direct consequence of the work of Jean BouRGAIN, Haïm BREZIS and NGUYÊN Hoài-Minh [5, lemma 2.1].

When $f \in W^{s, p}\left(\mathbb{S}^{m}, \mathbb{R}^{v}\right)$ with $s \in(0,1)$ and $s p=m$, the assertion (4.1) in proposition 4.1 with $\varepsilon=\frac{\delta}{2}$ implies that

$$
\mu_{\mathbb{H}^{m+1}}\left(\left\{x \in \mathbb{H}^{m+1} \mid \operatorname{dist}\left(F(x), f\left(\mathbb{S}^{m}\right)\right) \geq \delta\right\}\right) \leq \frac{C_{1}}{\delta^{p}} \iint_{\mathbb{S}^{m} \times \mathbb{S}^{m}} \frac{|f(y)-f(x)|^{p}}{|y-x|^{2 m}} \mathrm{~d} y \mathrm{~d} x
$$

This inequality (4.3) can be obtained when $s+\frac{1}{p}=s\left(1+\frac{1}{m}\right)<1$ by combining the classical extension $F \in$ $W^{s+1 / p, p}\left(\mathbb{B}^{m+1}, \mathbb{R}^{v}\right)$ of $f \in W^{s, p}\left(\mathbb{S}^{m}, \mathbb{R}^{v}\right)$ for linear fractional Sobolev spaces together with a fractional Hardy 
inequality [15, theorem 1.1] applied to the function $G=\operatorname{dist}\left(F, f\left(\mathbb{S}^{m}\right)\right) \in W_{0}^{s+1 / p, p}\left(\mathbb{B}^{m+1}, \mathbb{R}^{v}\right)$ :

$$
\begin{aligned}
\int_{\mathbb{B}^{m+1}} \frac{|G(x)|^{p}}{(1-|x|)^{m+1}} \mathrm{~d} x & \leq C_{2} \iint_{\mathbb{B}^{m+1} \times \mathbb{B}^{m+1}} \frac{|G(y)-G(x)|^{p}}{|y-x|^{2 m+2}} \mathrm{~d} y \mathrm{~d} x \\
& \leq C_{2} \iint_{\mathbb{B}^{m+1} \times \mathbb{B}^{m+1}} \frac{|F(y)-F(x)|^{p}}{|y-x|^{2 m+2}} \mathrm{~d} y \mathrm{~d} x \\
& \leq C_{3} \iint_{\mathbb{S}^{m} \times \mathbb{S}^{m}} \frac{|f(y)-f(x)|^{p}}{|y-x|^{2 m}} \mathrm{~d} y \mathrm{~d} x ;
\end{aligned}
$$

the estimate (4.3) follows then from the classical Chebyshev inequality.

The proofs of the counterpart of theorem 1.3 for $W^{1 / 2,2}\left(\mathbb{S}^{1}, \mathcal{N}\right)[30], W^{1, m}\left(\mathbb{S}^{m}, \mathcal{N}\right)[14]$ and $W^{1-1 / m, m}\left(\mathbb{S}^{m}, \mathcal{N}\right)$ [34], rely on a compactness argument on an extension of the map and do not explicitly estimate singular sets as in proposition 4.1.

The proof of proposition 4.1 follows the strategy of Jean Bourgain, Haïm BREzis, Petru MironEsCu and NGUYÊN Hoài-Minh [4, lemma 1.3; 5]. Since in the sequel we will work with the Poincaré ball model of the hyperbolic space, the proof uses the hyperharmonic extension as in $[14,48]$; this construction corresponds to the harmonic extension in the two-dimensional case $m+1=2[30, \S 2]$ and to the biharmonic extension when $m+1=4$ [47].

Proof of proposition 4.1. We define the function $F: \mathbb{B}^{m+1} \rightarrow \mathbb{R}^{v}$ to be the hyperharmonic extension of the function $f$, defined for each $z \in \mathbb{B}^{m+1}$ by $[1, \S \mathrm{V}]$

$$
F(z) \triangleq\left(1-|z|^{2}\right)^{m} f_{\mathbb{S}^{m}} \frac{f(y)}{|z-y|^{2 m}} \mathrm{~d} y=\frac{\left(1-|z|^{2}\right)^{m}}{\left|\mathbb{S}^{m}\right|} \int_{\mathbb{S}^{m}} \frac{f(y)}{|z-y|^{2 m}} \mathrm{~d} y .
$$

The hyperharmonic extension is equivariant under the action of the conformal transformations of the ball and of the sphere, both corresponding to the group of $(m+1)$-dimensional Möbius transformation preserving the unit ball: if $T: \mathbb{B}^{m+1} \rightarrow \mathbb{B}^{m+1}$ is a conformal transformation, then $F \circ T$ is the hyperharmonic extension of $f \circ T$.

The assertion (4.1) holds since by conformal invariance for every $z \in \mathbb{B}^{m+1}$,

$$
\frac{\left(1-|z|^{2}\right)^{m}}{\left|\mathbb{S}^{m}\right|} \int_{\mathbb{S}^{m}} \frac{1}{|z-y|^{2 m}} \mathrm{~d} y=1 .
$$

In order to prove the assertion (4.1), we first note that the Möbius transformations preserving the ball are exactly the isometries of the hyperbolic space in the Poincare disk model $[1, \S \mathrm{II}]$, and thus, in view of the equivariance of the hyperharmonic extension, it is sufficient to consider the case $z=0$. We have then for every $x \in \mathbb{S}^{m}$

$$
D F(0)=2 m f_{\mathbb{S}^{m}} f(y) \otimes y \mathrm{~d} y=2 m f_{\mathbb{S}^{m}}(f(y)-f(x)) \otimes y \mathrm{~d} y,
$$

since $\int_{\mathbb{S}^{m}} y \mathrm{~d} y=0$, and thus

$$
|D F(0)|_{\mathbb{H}^{m+1}}=\frac{1}{2}|D F(0)| \leq \underset{\mathbb{S}^{m}}{\operatorname{osc} f} .
$$

For the assertion (4.1), we observe that for every $x \in \mathbb{S}^{m}$ and $r \in[0,1)$, we have by (4.4)

$$
\operatorname{dist}\left(F(r x), f\left(\mathbb{S}^{n}\right)\right) \leq|F(r x)-f(x)| \leq\left(1-r^{2}\right)^{m} f_{\mathbb{S}^{m}} \frac{|f(y)-f(x)|}{|y-r x|^{2 m}} \mathrm{~d} y .
$$

We deduce therefrom that for every $\varepsilon>0$

$$
\operatorname{dist}\left(F(r x), f\left(\mathbb{S}^{n}\right)\right) \leq \varepsilon+\left(1-r^{2}\right)^{m} f_{\mathbb{S}^{m}} \frac{(|f(y)-f(x)|-\varepsilon)_{+}}{|y-r x|^{2 m}} \mathrm{~d} y .
$$


We next observe that, by the triangle inequality, for every $x, y \in \mathbb{S}^{m}$ and $r \in[0,1)$, we have

$$
|y-x| \leq|y-r x|+|r x-x|=|y-r x|+|y|-|r x| \leq 2|y-r x| .
$$

Therefore, we have

$$
\operatorname{dist}\left(F(r x), f\left(\mathbb{S}^{n}\right)\right) \leq \varepsilon+\frac{\left(1-r^{2}\right)^{m} 4^{m}}{\left|\mathbb{S}^{m}\right|} \int_{\mathbb{S}^{m}} \frac{(|f(y)-f(x)|-\varepsilon)_{+}}{|y-x|^{2 m}} \mathrm{~d} y .
$$

We define the set

$$
A_{\delta} \triangleq\left\{z \in \mathbb{B}^{m+1} \mid \operatorname{dist}\left(F(z), f\left(\mathbb{S}^{m}\right)\right) \geq \delta\right\}
$$

For each $x \in \mathbb{S}^{m}$, we set

$$
\rho_{\delta}(x) \triangleq \sup \left\{r \in[0,1) \mid r x \in A_{\delta}\right\},
$$

(with the convention that $\rho_{\delta}(x) \triangleq 0$ if $r x \notin A_{\delta}$ for every $r \in[0,1)$ ) and, since $m \geq 1$, we compute that

$$
\begin{aligned}
\mu_{\mathbb{H}^{m+1}}\left(A_{\delta}\right) & \leq \int_{\mathbb{S}^{m}} \int_{0}^{\rho_{\delta}(x)} \frac{2^{m+1} r^{m}}{\left(1-r^{2}\right)^{m+1}} \mathrm{~d} r \mathrm{~d} x \\
& \leq \int_{\mathbb{S}^{m}} \int_{0}^{\rho_{\delta}(x)} \frac{2^{m+1} r}{\left(1-r^{2}\right)^{m+1}} \mathrm{~d} r \mathrm{~d} x \leq \int_{\mathbb{S}^{m}} \frac{2^{m}}{m\left(1-\rho_{\delta}(x)^{2}\right)^{m}} \mathrm{~d} x .
\end{aligned}
$$

Since $\rho_{\delta}(x) x \in A_{\delta}$, we deduce from (4.5) that

$$
\frac{1}{\left(1-\rho_{\delta}(x)^{2}\right)^{m}} \leq \frac{4^{m}}{\left|\mathbb{S}^{m}\right|(\delta-\varepsilon)} \int_{\mathbb{S}^{m}} \frac{(|f(y)-f(x)|-\varepsilon)_{+}}{|y-x|^{2 m}} \mathrm{~d} y
$$

and we conclude that

$$
\mu_{\mathbb{H}^{m+1}}\left(A_{\delta}\right) \leq \frac{8^{m}}{m\left|\mathbb{S}^{m}\right|(\delta-\varepsilon)} \iint_{\mathbb{S}^{m} \times \mathbb{S}^{m}} \frac{(|f(y)-f(x)|-\varepsilon)_{+}}{|y-x|^{2 m}} \mathrm{~d} y \mathrm{~d} x .
$$

Remark 4.2. The proof of proposition 4.1 controls in fact the hyperbolic volume of the star-shaped hull $A_{\delta}^{\star, 0}$ of the set $A_{\delta}$ with respect to 0 defined as the smallest subset which is starshaped with respect to 0 and contains $A_{\delta}$. By invariance under the Möbius group that models the isometries of the hyperbolic space in the Poincare ball model, the volume of the starshaped hull $A_{\delta}^{\star, x}$ of the set $A_{\delta}$ with respect to any $x \in \mathbb{H}^{m+1}$ is also controlled.

\subsection{Ball merging}

Our second tool for proving theorem 1.3 is a construction that merges balls in a covering.

Lemma 4.3 (Merging balls on manifold). Let $\mathcal{X}$ be a Riemannian manifold. Given a positive integer $\ell \in$ $\mathbb{N}_{*}$, points $a_{1}, a_{2}, \ldots, a_{\ell} \in \mathcal{M}$ and radii $r_{1}, r_{2}, \ldots, r_{\ell} \in(0,+\infty)$, there exists $\ell^{\prime} \in\{1,2, \ldots, \ell\}$, points $a_{1}^{\prime}, \ldots, a_{\ell}^{\prime} \in \mathcal{M}$ and radii $r_{1}^{\prime}, \ldots, r_{\ell}^{\prime}$ such that

$$
\bigcup_{i=1}^{\ell} B_{r_{i}}^{\mathcal{X K}}\left(a_{i}\right) \subseteq \bigcup_{i=1}^{\ell^{\prime}} B_{r_{i}^{\prime}}^{\mathcal{M}}\left(a_{i}^{\prime}\right) \quad \text { and } \quad \sum_{i=1}^{\ell^{\prime}} r_{i}^{\prime} \leq \sum_{i=1}^{\ell} r_{i},
$$

and for every $i, j \in\{1,2, \ldots, \ell\}$ such that $i \neq j, \bar{B}_{r_{i}^{\prime}}^{\mathcal{X}}\left(a_{i}^{\prime}\right) \cap \bar{B}_{r_{j}^{\prime}}^{\mathcal{X l}}\left(a_{j}^{\prime}\right)=\emptyset$.

Lemma 4.3 merges a covering by balls into a covering by disjoint balls, while keeping the sums of the radii under control. This lemma is classical in the Euclidean case where it was a key tool lower bounds for GinzburgLandau energies [29, lemma 3.1; 51, proof of theorem 1; 52, lemma 4.1]. 
Proof of lemma 4.3. We proceed by induction. The lemma holds trivially when $\ell=1$. We assume now that $\ell>1$ and that the conclusion holds for $\ell-1$.

If for every $i, j \in\{1,2, \ldots, \ell\}$, we have $\bar{B}_{r_{i}}^{\text {\M }}\left(a_{i}\right) \cap \bar{B}_{r_{j}}^{\mathcal{M}}\left(a_{j}\right)=\emptyset$, the lemma is proved by taking $\ell^{\prime}=\ell$, and, for each $i \in\{1,2, \ldots, \ell\}, a_{i}^{\prime}=a_{i}$ and $r_{i}^{\prime}=r_{i}$.

Otherwise, we can assume without loss of generality that $\bar{B}_{r_{\ell-1}}^{\mathcal{X K}}\left(a_{\ell-1}\right) \cap \bar{B}_{r_{\ell}}^{\mathcal{X K}}\left(a_{\ell}\right) \neq \emptyset$. By the triangle inequality, this implies that $d_{\mathcal{M}}\left(a_{\ell-1}, a_{\ell}\right) \leq r_{\ell-1}+r_{\ell}$. Since the distance $d_{\mathcal{M}}$ is a geodesic distance on the manifold $\mathcal{M}$, there exists a point $\tilde{a}_{\ell-1} \in \mathcal{M}$ such that

$$
d_{\mathcal{M}}\left(\tilde{a}_{\ell-1}, a_{\ell-1}\right)=\frac{1}{2}\left(d_{\mathcal{M}}\left(a_{\ell-1}, a_{\ell}\right)+r_{\ell}-r_{\ell-1}\right),
$$

and

$$
d_{\mathcal{M}}\left(\tilde{a}_{\ell-1}, a_{\ell}\right)=\frac{1}{2}\left(d_{\mathcal{M}}\left(a_{\ell-1}, a_{\ell}\right)+r_{\ell-1}-r_{\ell}\right) .
$$

We now set $\tilde{r}_{\ell-1} \triangleq \frac{1}{2}\left(d_{\mathcal{M}}\left(a_{\ell-1}, a_{\ell}\right)+r_{\ell-1}+r_{\ell}\right)$. We observe that

$$
\tilde{r}_{\ell-1} \leq r_{\ell-1}+r_{\ell} \quad \text { and } \quad B_{r_{\ell-1}}^{\mathcal{M}}\left(a_{\ell-1}\right) \cup B_{r_{\ell}}^{\mathcal{M}}\left(a_{\ell}\right) \subseteq B_{\tilde{r}_{\ell-1}}^{\mathcal{X}}\left(\tilde{a}_{\ell-1}\right) .
$$

We set, for $i \in\{1,2, \ldots, \ell-2\}, \tilde{a}_{i} \triangleq a_{i}$ and $\tilde{r}_{i} \triangleq r_{i}$ and $\tilde{\ell} \triangleq \ell-1$. We conclude by applying our induction hypothesis to $\tilde{\ell}, \tilde{a}_{1}, \tilde{a}_{2}, \ldots, \tilde{a}_{\tilde{\ell}}$ and $\tilde{r}_{1}, \tilde{r}_{2}, \ldots, \tilde{r}_{\tilde{\ell}}$.

Remark 4.4. The proof of lemma 4.3 relies on the fact that $\mathcal{M}$ is a geodesic metric space to construct the point $\tilde{a}_{\ell-1}$. If $\mathcal{M}$ is merely a length metric space, the proof gives weaker forms of lemma 4.3 where either the inequality on the sum of radius holds up to an arbitrary error $\varepsilon>0$ or the open balls, instead of the closed balls, are disjoint. If $\mathcal{M}$ is simply a metric space, than we can still take $\tilde{a}_{\ell-1} \in \bar{B}_{r_{\ell-1}}\left(a_{\ell-1}\right) \cap \bar{B}_{r_{\ell}}\left(a_{\ell}\right)$ and $\tilde{r}_{\ell-1}=2 \max \left\{r_{\ell-1}, r_{\ell}\right\}$ and obtain the conclusion with an additional unbounded $2^{\ell-1}$ factor multiplying the sum of radii on the right-hand side.

We will also rely on a straightforward characterization of the geometry of hyperbolic spheres [16, §III.5].

Lemma 4.5 (Description of spheres in the hyperbolic space). For every $\rho>0$ and every $m \in \mathbb{N}$, the hyperbolic sphere $\partial B_{\rho}^{\mathbb{H}^{m+1}}(a)$ is isometric to the Euclidean $\mathbb{S}_{\text {sinh } \rho}^{m}$.

Proof. We work in the Poincaré ball model and assume without loss of generality that $a=0 \in \mathbb{B}^{m+1} \simeq \mathbb{H}^{m+1}$. The hyperbolic ball $B_{\rho}^{\mathbb{H}^{m+1}}(0)$ is modeled by the Euclidean ball $B_{\tanh \frac{\rho}{2}}(0)$. The Poincaré metric at every point $x \in \partial B_{\tanh \frac{\rho}{2}}(0)$ on this sphere is $\sqrt{g_{x}(v)}=2|v| /\left(1-\left(\tanh \frac{\rho}{2}\right)^{2}\right)=2\left(\cosh \frac{\rho}{2}\right)^{2}|v|$ which means that the radius of the isometric Euclidean sphere is $2\left(\cosh \frac{\rho}{2}\right)^{2} \tanh \frac{\rho}{2}=\sinh \rho$.

\subsection{Proof of the theorem}

Theorem 1.3 will follow from the following slightly stronger statement, involving a truncated fractional integral.

Theorem 4.6 (Estimate on free homotopy decomposition by a truncated fractional energy). If $\varepsilon>0$ is small enough, there is a constant $C>0$ such that for every $\lambda>0$, there exists a finite set $\mathscr{F}^{\lambda} \subset \mathscr{C}\left(\mathbb{S}^{m}, \mathcal{N}\right)$ such that any map $f \in \mathscr{C}\left(\mathbb{S}^{m}, \mathcal{N}\right)$ satisfying

$$
\iint_{\mathbb{S}^{m} \times \mathbb{S}^{m}} \frac{\left(d_{\mathcal{N}}(f(y), f(x))-\varepsilon\right)_{+}}{|y-x|^{2 m}} \mathrm{~d} x \mathrm{~d} y \leq \lambda,
$$

has a free homotopy decomposition into $f_{1}, \ldots, f_{k} \in \mathscr{F}^{\lambda}$ with $k \leq C \lambda$. 
Proof of theorem 4.6. We apply proposition 4.1 to $f$. We define for each $\delta>0$ the sets $\mathcal{N}_{\delta} \triangleq\{y \in$ $\left.\mathbb{R}^{v} \mid \operatorname{dist}(y, \mathcal{N})<\delta\right\}$ and

$$
A_{\delta} \triangleq F^{-1}\left(\mathbb{R}^{v} \backslash \mathcal{N}_{\delta}\right)=\left\{x \in \mathbb{H}^{m+1} \mid \operatorname{dist}(F(x), \mathcal{N}) \geq \delta\right\} .
$$

Since $\mathcal{N}$ is a smooth submanifold of $\mathbb{R}^{v}$, there exists $\delta_{\star}>0$ and a Lipschitz-continuous retraction $\Pi: \mathcal{N}_{\delta_{\star}} \rightarrow \mathcal{N}$, that is, one has for every $y \in \mathcal{N}_{\delta_{*}}, \Pi(y) \in \mathcal{N}$ and for every $y \in \mathcal{N}, \Pi(y)=y$.

By the estimate (4.1) in proposition 4.1, we observe that if $a \in A_{\delta_{*}}$, then for every $x \in \mathbb{H}^{m+1}$ we have

$$
F(x) \geq \delta_{\star}-m \operatorname{diam}(\mathcal{N}) d_{\mathbb{H}^{m+1}}(x, a),
$$

If we take $\rho \triangleq \frac{\delta_{\star}}{2 m \operatorname{diam}(\mathcal{N})}$, we have

$$
\bar{B}_{\rho}^{\mathbb{H}^{m+1}}(a) \subseteq A_{\delta_{\star} / 2}
$$

We consider now a maximal set of points $A \subseteq A_{\delta_{\star}}$ such that if $a, b \in A$ and $a \neq b$ then $d_{\mathbb{H}^{m+1}}(a, b) \geq 2 \rho$. By construction, we have

$$
A_{\delta_{\star}} \subseteq \bigcup_{a \in A} B_{2 \rho}^{\mathbb{H}^{m+1}}(a)
$$

On the other hand the balls $\left(B_{\rho}^{\mathbb{H}^{m+1}}(a)\right)_{a \in A}$ are disjoint and thus by (4.7), we have

$$
\sum_{a \in A} \mu_{\mathbb{H}^{m+1}}\left(B_{\rho}^{\mathbb{H}^{m+1}}(a)\right) \leq \mu_{\mathbb{H}^{m+1}}\left(A_{\delta_{\star} / 2}\right)
$$

By the invariance of the volume of balls in the hyperbolic space, we deduce that

$$
\# A \leq C_{1} \iint_{\mathbb{S}^{m} \times \mathbb{S}^{m}} \frac{(|f(y)-f(x)|-\varepsilon)_{+}}{|y-x|^{2 m}} \mathrm{~d} y \mathrm{~d} x \leq C_{1} \lambda .
$$

By lemma 4.3, we obtain the existence of $k \in\{1, \ldots, \# A\}, a_{1}, \ldots, a_{k} \in \mathbb{H}^{m+1}$ and $\rho_{1}, \ldots, \rho_{k} \in\left[2 \rho, 2 C_{1} \lambda \rho\right]$ such that if $i, j \in\{1, \ldots, k\}$ and $i \neq j$, then $\bar{B}_{\rho_{i}}^{\mathbb{H}^{m+1}}\left(a_{i}\right) \cap \bar{B}_{\rho_{j}}^{\mathbb{H}^{m+1}}\left(a_{j}\right)=\emptyset$ and

$$
A_{\delta_{\star}} \subseteq \bigcup_{i=1}^{k} \bar{B}_{\rho_{i}}^{\mathbb{H}^{m+1}}\left(a_{i}\right)
$$

We conclude by defining the set

$$
U \triangleq \mathbb{H}^{m+1} \backslash \bigcup_{i=1}^{k} B_{\rho_{i}}^{\mathbb{H}^{m+1}}\left(a_{i}\right)
$$

and the map

$$
\left.\tilde{F} \triangleq \Pi \circ F\right|_{U}: U \rightarrow N .
$$

We observe that $\partial U \cap \mathbb{B}^{m+1}=\bigcup_{i=1}^{k} \partial B_{\rho_{i}}^{\mathbb{H}^{m+1}}\left(a_{i}\right)$ and that for every $i \in\{1, \ldots, k\}$, the set $\partial B_{\rho_{i}}^{\mathbb{H}^{m+1}}\left(a_{i}\right)$ is isometric by lemma 4.5 to a Euclidean $m$-dimensional sphere of radius $\sinh \rho_{i}$. This implies then that for every $i \in$ $\{1, \ldots, k\}$, the map $\left.\tilde{F}\right|_{\partial B_{\rho_{i}}^{\mathbb{H}^{m+1}}\left(a_{i}\right)}$ is homotopic on $\partial B_{\rho_{i}}^{\mathbb{H}^{m+1}}\left(a_{i}\right) \simeq \mathbb{S}^{m}$ to some map $g_{i}: \mathbb{S}^{m} \rightarrow \mathcal{N}$ whose Lipschitz constant is controlled by $C_{2} \sinh \left(C_{3} \lambda\right)$. By the Ascoli compactness theorem, there exists a finite set of maps $\mathscr{F}^{\lambda} \subset \mathscr{C}\left(\mathbb{S}^{m}, \mathbb{S}^{m}\right)$ such that any map from $\mathbb{S}^{m}$ to $\mathcal{N}$ whose Lipschitz constant does not exceed $C_{2} \sinh \left(C_{3} \lambda\right)$ is homotopic to some map in $\mathscr{F}^{\lambda}$. In particular, for every $i \in\{1, \ldots, k\}$, there exists a map $f_{i} \in \mathscr{F}^{\lambda}$ which is homotopic to $g_{i}$ on $\mathbb{S}^{m}$ and thus to $\left.\tilde{F}\right|_{\partial B_{\rho_{i}}^{\mathbb{m}^{m+1}}\left(a_{i}\right)}$ on $\mathbb{S}^{m} \simeq \partial B_{\rho_{i}}^{\mathbb{H}^{m+1}}\left(a_{i}\right)$.

We consider now a ball $B_{2 \rho}^{\mathbb{H}^{m+1}}\left(a_{\star}\right) \subset \tilde{U}$ and a map $\breve{F} \in \mathscr{C}(U, \mathcal{N})$ such that $\breve{F}=\tilde{F}$ in $U \backslash B_{2 \rho}^{\mathbb{H}^{m+1}}\left(a_{\star}\right)$ and $\breve{F}$ is constant on $B_{\rho}^{\mathbb{H}^{m+1}}\left(a_{\star}\right)$. We now consider maps $\Phi_{i}: \overline{\mathbb{B}}^{m+1} \rightarrow U \backslash B_{\rho}\left(a_{\star}\right)$ such that for every $i \in\{1, \ldots, k\}$, $\Phi_{i}$ is injective, $B_{\rho_{i}}^{\mathbb{H}^{m+1}}\left(a_{i}\right) \subset \Phi_{i}\left(\mathbb{B}^{m+1}\right) \backslash \bigcup_{j \neq i} \Phi_{j}\left(\mathbb{B}^{m+1}\right)$ and $\Phi_{i}\left(\overline{\mathbb{B}}^{m+1}\right) \cap \partial B_{\rho}^{\mathbb{H}^{m+1}}\left(a_{\star}\right)$ is a nondegenerate geodesic ball in $\partial B_{\rho}^{\mathbb{H}^{m+1}}\left(a_{\star}\right)$. We define $\breve{U}=U \backslash\left(B_{\rho}\left(a_{\star}\right) \cup \bigcup_{i=1}^{k} \Phi_{i}\left(\mathbb{B}^{m+1}\right)\right) \subset \mathbb{H}^{m+1}$, and we observe that $\partial \breve{U} \cap \mathbb{B}^{m+1}$ is homeomorphic to $\mathbb{S}^{m+1}$ and that $\left.\breve{F}\right|_{\partial \breve{U} \cap \mathbb{B}^{m+1}}$ has a free homotopy decomposition into $f_{1}, \ldots, f_{k}$, and hence by homotopy invariance, $f$ also has a free homotopy decomposition into $f_{1}, \ldots, f_{k}$. 
We deduce now theorem 1.3 from theorem 4.6.

Proof of theorem 1.3. We note that, since the $\operatorname{map} f: \mathbb{S}^{m} \rightarrow \mathcal{N}$ is bounded, we have

$$
\iint_{\mathbb{S}^{m} \times \mathbb{S}^{m}} \frac{(|f(y)-f(x)|-\varepsilon)_{+}}{|y-x|^{2 m}} \mathrm{~d} y \mathrm{~d} x \leq \operatorname{diam}(\mathcal{N}) \underset{\substack{(x, y) \in \mathbb{S}^{m} \times \mathbb{S}^{m} \\|f(y)-f(x)|>\varepsilon}}{\iint^{2 m}} \frac{1}{|y-x|^{2 m}} \mathrm{~d} y \mathrm{~d} x,
$$

and the conclusion then follows from theorem 4.6.

We will observe in the sequel that when $m \geq 2$, an estimate of the form (4.8) holds without any boundedness assumption on the map $f$ and with a constant of the order of $\varepsilon$ (see proposition 5.5 below).

Proof of theorem 1.4. This follows from theorem 1.3 and proposition 2.3.

\subsection{Proof of the length estimate}

In order to prove theorem 1.5 , we will prove a slightly stronger inequality.

Theorem 4.7 (Estimate on free homotopy decomposition by a truncated fractional energy). If $\varepsilon>0$ is small enough, there is a constant $C>0$ such that every $f \in \mathscr{C}(\mathbb{S}, \mathcal{N})$ has a free decomposition into $f_{1}, \ldots, f_{k} \in$ $\mathscr{C}^{1}\left(\mathbb{S}^{1}, \mathcal{N}\right)$ such that

$$
\sum_{i=1}^{k} \int_{\mathbb{S}^{1}}\left|f_{i}^{\prime}\right| \leq C \iint_{\mathbb{S}^{1} \times \mathbb{S}^{1}} \frac{\left(d_{\mathcal{N}}(f(y), f(x))-\varepsilon\right)_{+}}{|y-x|^{2}} \mathrm{~d} x \mathrm{~d} y .
$$

Proof. We begin as in the proof of theorem 4.6, by an application of proposition 4.1 to $f$, the definition for each $\delta>0$ of the sets $\mathcal{N}_{\delta} \triangleq\left\{y \in \mathbb{R}^{v} \mid \operatorname{dist}(y, \mathcal{N})<\delta\right\}$ and $A_{\delta} \triangleq F^{-1}\left(\mathbb{R}^{v} \backslash \mathcal{N}_{\delta}\right)$ and the existence of $\delta \star$ ensuring a Lipschitz-continuous retraction $\Pi: \mathcal{N}_{\delta_{*}} \rightarrow \mathcal{N}$. By the estimate (4.1) in proposition 4.1, we observe that if $\rho \triangleq \frac{\delta_{\star}}{2 \operatorname{diam}(\mathcal{N})}$ then for every $a \in A_{\delta_{\star}}$, then for every $x \in \mathbb{H}^{2}$, we have

$$
\bar{B}_{\rho}^{\mathbb{H}}{ }^{2}(a) \subseteq A_{\delta \star / 2}
$$

We take $A \subseteq A_{\delta_{\star}}$ to be a maximal set of points such that if $a, b \in A$ and $a \neq b$ then $d_{\mathbb{H}^{2}}(a, b) \geq 2 \rho$. In particular, this implies that

$$
A_{\delta_{\star}} \subseteq \bigcup_{a \in A} B_{2 \rho}^{\mathbb{H}^{2}}(a)
$$

Since the balls $\left(B_{\rho}^{\mathbb{H}^{2}}(a)\right)_{a \in A}$ are disjoint, thus by (4.9) and the invariance of the volume of planes, in the hyperbolic space we deduce that

$$
\# A \leq C_{1} \sum_{a \in A} \mu_{\mathbb{H}^{2}}\left(B_{\rho}^{\mathbb{H}^{2}}(a)\right) \leq C_{2} \mu_{\mathbb{H}^{2}}\left(A_{\delta_{\star} / 2}\right) .
$$

We partition now the finite set $A$ into the finite sets $A_{1}, \ldots, A_{k}$ in such a way that for every $i \in\{1, \ldots, k\}$ the sets $\bigcup_{a \in A_{1}} \bar{B}_{2 \rho}^{\mathbb{H}^{2}}(a), \ldots, \bigcup_{a \in A_{k}} \bar{B}_{2 \rho}^{\mathbb{H}^{2}}(a)$ are disjoint connected sets. We define for each $i \in\{1, \ldots, k\}$ the compact set $K_{i} \subset \mathbb{H}^{2}$ as the set points in $\mathbb{H}^{2}$ that are not connected in $\mathbb{H}^{2} \backslash \bigcup_{a \in A_{i}} \bar{B}^{\mathbb{H}^{2}}$ to $\mathbb{S}^{1} \simeq \partial \mathbb{B}^{2}$. We conclude by observing that $\partial K_{i} \notin A_{\delta_{\star}}$, that $\partial K_{i}$ is a union of arcs of circle and that

$$
\int_{\partial K_{i}}|D F| \leq C_{3} \# A_{i}
$$

Proof of theorem 1.5. This follows from theorem 4.7, in view of (4.8). 


\section{Scaling and comparison of truncated fractional energies}

In this section we improve the estimate of theorem 1.3 into an estimate that scales optimally with respect to $\varepsilon$ as $\varepsilon \rightarrow 0$. Our results are the counterpart of NGUYÊN Hoài-Minh's estimates on the topological degree [43], but are obtained with a different strategy.

\subsection{Scaling of truncated fractional energies}

In order to improve the estimate of theorem 1.3, we first study how truncated fractional integral scale with varying values of the truncation in the next proposition.

Proposition 5.1 (Scaling of truncated fractional energies on a convex set). For every $p \in[0,+\infty)$ and every $m \in \mathbb{N}$, there exists a constant $C>0$ such that for every convex set $\Omega \subset \mathbb{R}^{m}$ and for every map $f: \Omega \rightarrow \mathcal{N}$, if $\delta<\varepsilon$ one has

$$
\iint_{(x, y) \in \Omega \times \Omega d_{\mathcal{N}}(f(y), f(x)) \geq \varepsilon} \frac{\left(d_{\mathcal{N}}(f(y), f(x))-\varepsilon\right)^{p}}{|y-x|^{2 m}} \mathrm{~d} y \mathrm{~d} x \leq C\left(\frac{\delta}{\varepsilon}\right)^{m-1-(p-1)_{+}} \iint_{\substack{(x, y) \in \Omega \times \Omega \\ d_{\mathcal{N}}(f(y), f(x)) \geq \delta}} \frac{\left(d_{\mathcal{N}}(f(y), f(x))-\delta\right)^{p}}{|y-x|^{2 m}} \mathrm{~d} y \mathrm{~d} x .
$$

When either $1 \leq p<m$, or $p \leq 1$ and $m>2$, then $m-1>(p-1)_{+}$and the estimate of proposition 5.1 improves the straightforward monotonicity estimate: if $\delta \leq \varepsilon$, then

$$
\iint_{\substack{(x, y) \in \Omega \times \Omega \\ d_{\mathcal{N}}(f(y), f(x)) \geq \varepsilon}} \frac{\left(d_{\mathcal{N}}(f(y), f(x))-\varepsilon\right)^{p}}{|y-x|^{2 m}} \mathrm{~d} y \mathrm{~d} x \leq \iint_{\substack{(x, y) \in \Omega \times \Omega) \\ d_{\mathcal{N}}(f(y), f(x)) \geq \delta}} \frac{\left(d_{\mathcal{N}}(f(y), f(x))-\delta\right)^{p}}{|y-x|^{2 m}} \mathrm{~d} y \mathrm{~d} x .
$$

If the set $\Omega \subset \mathbb{R}^{m}$ is bounded and if the map $f: \Omega \rightarrow \mathbb{R}^{m}$ is the identity, one has

$$
\begin{aligned}
\iint_{\substack{(x, y) \in \Omega \times \Omega \\
d_{\mathcal{N}}(f(y), f(x)) \geq \varepsilon}} \frac{\left(d_{\mathcal{N}}(f(y), f(x))-\varepsilon\right)^{p}}{|y-x|^{2 m}} \mathrm{~d} y \mathrm{~d} x & =\iint_{\substack{(x, y) \in \Omega \times \Omega \\
|y-x| \geq \varepsilon}} \frac{(|y-x|-\varepsilon)^{p}}{|y-x|^{2 m}} \mathrm{~d} y \mathrm{~d} x \\
& \simeq \int_{\varepsilon}^{1} \frac{(r-\varepsilon)^{p}}{r^{m+1}} \mathrm{~d} r=\frac{1}{\varepsilon^{m-p}} \int_{0}^{\frac{1}{\varepsilon}-1} \frac{t^{p}}{(t+1)^{m+1}} \mathrm{~d} t \\
& \simeq \begin{cases}\frac{1}{\varepsilon^{m-p}} & \text { if } p<m, \\
\ln \frac{1}{\varepsilon} & \text { if } p=m, \\
1 & \text { if } p>m,\end{cases}
\end{aligned}
$$

as $\varepsilon \rightarrow 0$, by the change of variables $r=\varepsilon(t+1)$. This computation means that the scaling estimate of proposition 5.1 is optimal when $1 \leq p<m$. We do not know whether the estimate can be improved when $0 \leq p<1$ (see open problem 3 below). The estimate will already appear to be strong enough to obtain some comparison between truncated fractional integrals of different exponents in proposition 5.5 below.

Proof of proposition 5.1. By the triangle inequality, we have

$$
\begin{aligned}
& \iint_{\substack{(x, y) \in \Omega \times \Omega \\
d_{\mathcal{N}}(f(y), f(x)) \geq \varepsilon}} \frac{\left(d_{\mathcal{N}}(f(y), f(x))-\varepsilon\right)^{p}}{|y-x|^{2 m}} \mathrm{~d} y \mathrm{~d} x \\
& \quad \leq 2^{(p-1)_{+}} \iint_{\substack{(x, y) \in \Omega \times \Omega \\
d_{\mathcal{N}}\left(f(y), f\left(\frac{x+y}{2}\right)\right) \geq \frac{\varepsilon}{2}}} \frac{\left(d_{\mathcal{N}}\left(f(y), f\left(\frac{x+y}{2}\right)\right)-\frac{\varepsilon}{2}\right)^{p}}{|y-x|^{2 m}} \mathrm{~d} y \mathrm{~d} x+2^{(p-1)_{+}} \iint_{\begin{array}{r}
(x, y) \in \Omega \times \Omega \\
d_{\mathcal{N}}\left(f\left(\frac{x+y}{2}\right), f(x)\right) \geq \frac{\varepsilon}{2}
\end{array}} \frac{\left(d_{\mathcal{N}}\left(f\left(\frac{x+y}{2}\right), f(x)\right)-\frac{\varepsilon}{2}\right)^{p}}{|y-x|^{2 m}} \mathrm{~d} y \mathrm{~d} x,
\end{aligned}
$$


and thus by symmetry under exchange of $x$ and $y$

$$
\iint_{\substack{(x, y) \in \Omega \times \Omega \\
d_{\mathcal{N}}(f(y), f(x)) \geq \varepsilon}} \frac{\left(d_{\mathcal{N}}(f(y), f(x))-\varepsilon\right)^{p}}{|y-x|^{2 m}} \mathrm{~d} y \mathrm{~d} x=2^{1+(p-1)_{+}} \iint_{\begin{array}{c}
(x, y) \in \Omega \times \Omega \\
d_{\mathcal{N}}\left(f\left(\frac{x+y}{2}\right), f(x)\right) \geq \frac{\varepsilon}{2}
\end{array}} \frac{\left(d_{\mathcal{N}}\left(f\left(\frac{x+y}{2}\right), f(x)\right)-\frac{\varepsilon}{2}\right)^{p}}{|y-x|^{2 m}} \mathrm{~d} y \mathrm{~d} x .
$$

We apply now the change of variable $y=2 z-x$ and we obtain

$$
\begin{aligned}
\iint_{\substack{(x, y) \in \Omega \times \Omega \\
d_{\mathcal{N}}\left(f\left(\frac{x+y}{2}\right), f(x)\right) \geq \frac{\varepsilon}{2}}} \frac{\left(d_{\mathcal{N}}\left(f\left(\frac{x+y}{2}\right), f(x)\right)-\frac{\varepsilon}{2}\right)^{p}}{|y-x|^{2 m}} \mathrm{~d} y \mathrm{~d} x & =\frac{1}{2^{m}} \int_{\Omega}\left(\int_{\Sigma_{x}} \frac{\left(d_{\mathcal{N}}(f(z), f(x))-\frac{\varepsilon}{2}\right)^{p}}{|z-x|^{2 m}} \mathrm{~d} z\right) \mathrm{d} x \\
& \leq \frac{1}{2^{m}} \iint_{\begin{array}{c}
(x, y) \in \Omega \times \Omega \\
d_{\mathcal{N}}(f(y), f(x)) \geq \frac{\varepsilon}{2}
\end{array}} \frac{\left(d_{\mathcal{N}}(f(y), f(x))-\frac{\varepsilon}{2}\right)^{p}}{|y-x|^{2 m}} \mathrm{~d} y \mathrm{~d} x,
\end{aligned}
$$

where for every $x \in \Omega$, we have defined the set

$$
\Sigma_{x} \triangleq\left\{z \in \Omega \mid 2 z-x \in \Omega \text { and } d_{\mathcal{N}}(f(z), f(x)) \geq \frac{\varepsilon}{2}\right\} .
$$

By combining the inequalities (5.2) and (5.3), we deduce that for every $\varepsilon>0$,

$$
\iint_{\substack{(x, y) \in \Omega \times \Omega \\
d_{\mathcal{N}}(f(y), f(x)) \geq \varepsilon}} \frac{\left(d_{\mathcal{N}}(f(y), f(x))-\varepsilon\right)^{p}}{|y-x|^{2 m}} \mathrm{~d} y \mathrm{~d} x \leq 2^{(p-1)_{+}-(m-1)} \iint_{\begin{array}{r}
(x, y) \in \Omega \times \Omega \\
d_{\mathcal{N}}(f(y), f(x)) \geq \frac{\varepsilon}{2}
\end{array}} \frac{\left(d_{\mathcal{N}}(f(y), f(x))-\frac{\varepsilon}{2}\right)^{p}}{|y-x|^{2 m}} \mathrm{~d} y \mathrm{~d} x .
$$

By iterating the estimate (5.4), we deduce that for every nonnegative integer $\ell \in \mathbb{N}$,

$$
\iint_{\substack{(x, y) \in \Omega \times \Omega \\
d_{\mathcal{N}}(f(y), f(x)) \geq \varepsilon}} \frac{\left(d_{\mathcal{N}}(f(y), f(x))-\varepsilon\right)^{p}}{|y-x|^{2 m}} \mathrm{~d} x \mathrm{~d} y \leq 2^{\ell\left((p-1)_{+}-(m-1)\right)} \iint_{\begin{array}{c}
(x, y) \in \Omega \times \Omega \\
d_{\mathcal{N}}(f(y), f(x)) \geq \frac{\varepsilon}{2^{\ell}}
\end{array}} \frac{\left(d_{\mathcal{N}}(f(y), f(x))-\frac{\varepsilon}{2^{\ell}}\right)^{p}}{|y-x|^{2 m}} \mathrm{~d} y \mathrm{~d} x .
$$

If $\delta \in(0, \varepsilon)$, we let $\ell \in \mathbb{N}$ be defined by the condition $2^{-(\ell+1)} \varepsilon \leq \delta<2^{-\ell} \varepsilon$ and we conclude that

$$
\iint_{\substack{(x, y) \in \Omega \times \Omega \\
d_{\mathcal{N}}(f(y), f(x)) \geq \varepsilon}} \frac{\left(d_{\mathcal{N}}(f(y), f(x))-\varepsilon\right)^{p}}{|y-x|^{2 m}} \mathrm{~d} y \mathrm{~d} x \leq\left(\frac{2 \delta}{\varepsilon}\right)^{m-1-(p-1)_{+}} \iint_{\begin{array}{r}
(x, y) \in \Omega \times \Omega \\
d_{\mathcal{N}}(f(y), f(x)) \geq \delta
\end{array}} \frac{\left(d_{\mathcal{N}}(f(y), f(x))-\delta\right)^{p}}{|y-x|^{2 m}} \mathrm{~d} y \mathrm{~d} x .
$$

In order to improve the statement of theorem 1.3, we will derive the counterpart of proposition 5.1 for spheres.

Proposition 5.2 (Scaling of truncated fractional energies on a sphere). For every $p \in[0,+\infty)$ and every $m \in$ $\mathbb{N}$, there exists a constant $C>0$ such that for every map $f: \mathbb{S}^{m} \rightarrow \mathcal{N}$, if $\delta<\varepsilon$ one has

$$
\iint_{\substack{(x, y) \in \mathbb{S}^{m} \times \mathbb{S}^{m} \\ d_{\mathcal{N}}(f(y), f(x) \geq \varepsilon}} \frac{\left(d_{\mathcal{N}}(f(y), f(x))-\varepsilon\right)^{p}}{|y-x|^{2 m}} \mathrm{~d} y \mathrm{~d} x \leq C\left(\frac{\delta}{\varepsilon}\right)^{m-1-(p-1)_{+}} \iint_{\substack{(x, y) \in \mathbb{S}^{m} \times \mathbb{S}^{m} \\ d_{\mathcal{N}}(f(y), f(x)) \geq \delta}} \frac{\left(d_{\mathcal{N}}(f(y), f(x))-\delta\right)^{p}}{|y-x|^{2 m}} \mathrm{~d} y \mathrm{~d} x .
$$

The proof of proposition 5.2 will rely on its counterpart on a convex set of the Euclidean space proposition 5.1 and on a suitable covering of the sphere by spherical caps.

Lemma 5.3 (Covering pairs of points of the sphere). Let $m \in \mathbb{N}_{\star}$. If $a_{0}, \ldots, a_{m+1} \in \mathbb{S}^{m} \subset \mathbb{R}^{m+1}$ are the vertices of a regular simplex, then for every $x, y \in \mathbb{S}^{m}$ there exists $i \in\{0, \ldots, m+1\}$ such that

$$
a_{i} \cdot x \geq-\frac{1}{\sqrt{m+1}} \quad \text { and } \quad a_{i} \cdot y \geq-\frac{1}{\sqrt{m+1}} .
$$


Proof. Let

$$
I=\left\{i \in\{0, \ldots, m+1\} \mid a_{i} \cdot x_{i}<-\frac{1}{\sqrt{m+1}}\right\} .
$$

We claim that $\# I<\frac{m}{2}+1$. We assume without loss of generality that $\# I>0$. We then have

$$
\sum_{i \in I} a_{i} \cdot x<-\frac{\# I}{\sqrt{m+1}}
$$

Since for every $i, j \in\{0,1, \ldots, m+1\}, a_{i} \cdot a_{j}=\frac{m+2}{m+1} \delta_{i j}-\frac{1}{m+1}$, where $\delta_{i j}$ denotes Kronecker's delta, we have

$$
\left|\sum_{i \in I} a_{i}\right|^{2}=\sum_{i \in I} \sum_{j \in I} a_{i} \cdot a_{j}=\frac{\# I(m+2-\# I)}{m+1} .
$$

Hence, by the Cauchy-Schwarz inequality we have, since $|x|=1$, by (5.5) and (5.6)

$$
\frac{(\# I)^{2}}{m+1}<\left(\sum_{i \in I} a_{i} \cdot x\right)^{2} \leq\left|\sum_{i \in I} a_{i}\right|^{2}|x|^{2}=\frac{\# I(m+2-\# I)}{m+1},
$$

so that $\# I<m+2-\# I$, and therefore $\# I<\frac{m}{2}+1$.

If we set similarly

$$
J=\left\{i \in\{0, \ldots, m+1\} \mid a_{i} \cdot y<-\frac{1}{\sqrt{m+1}}\right\},
$$

we obtain that $\# I+\# J<m+2$. Hence, there exists $i \in\{0,1, \ldots, m+1\} \backslash(I \cup J)$ and thus the conclusion holds by definition of the sets $I$ and $J$.

Proof of proposition 5.2. Let $a_{0}, \ldots, a_{m+1} \in \mathbb{S}^{m} \subset \mathbb{R}^{m+1}$ be the vertices of an equilateral simplex and define for each $i \in\{0,1, \ldots, m+1\}$, the spherical cap

$$
A_{i} \triangleq\left\{x \in \mathbb{S}^{m} \mid a_{i} \cdot x \geq-\frac{1}{\sqrt{m+1}}\right\} .
$$

In view of lemma 5.3, we have $\mathbb{S}^{m} \times \mathbb{S}^{m}=\bigcup_{i=0}^{m+1} A_{i} \times A_{i}$, and therefore

$$
\iint_{\substack{(x, y) \in \mathbb{S}^{m} \times \mathbb{S}^{m} \\
d_{\mathcal{N}}(f(y), f(x)) \geq \varepsilon}} \frac{\left(d_{\mathcal{N}}(f(y), f(x))-\varepsilon\right)^{p}}{|y-x|^{2 m}} \mathrm{~d} y \mathrm{~d} x \leq \sum_{i=0}^{m+1} \iint_{\begin{array}{c}
(x, y) \in A_{i} \times A_{i} \\
d_{\mathcal{N}}(f(y), f(x)) \geq \varepsilon
\end{array}} \frac{\left(d_{\mathcal{N}}(f(y), f(x))-\varepsilon\right)^{p}}{|y-x|^{2 m}} \mathrm{~d} y \mathrm{~d} x .
$$

Since for every $i \in\{0, \ldots, m+1\}$, the spherical cap $A_{i}$ is diffeomorphic to a ball of $\mathbb{R}^{m}$, we have in view of proposition 5.1,

$$
\begin{aligned}
& \iint_{\substack{(x, y) \in A_{i} \times A_{i} \\
d_{\mathcal{N}}(f(y), f(x)) \geq \varepsilon}} \frac{\left(d_{\mathcal{N}}(f(y), f(x))-\varepsilon\right)^{p}}{|y-x|^{2 m}} \mathrm{~d} y \mathrm{~d} x \\
& \leq C_{1}\left(\frac{\delta}{\varepsilon}\right)^{m-1-(p-1)_{+}} \iint_{\substack{(x, y) \in A_{i} \times A_{i} \\
d_{\mathcal{N}}(f(y), f(x)) \geq \delta}} \frac{\left(d_{\mathcal{N}}(f(y), f(x))-\delta\right)^{p}}{|y-x|^{2 m}} \mathrm{~d} y \mathrm{~d} x \\
& \leq C_{1}\left(\frac{\delta}{\varepsilon}\right)^{m-1-(p-1)_{+}} \iint_{\substack{(x, y) \in \mathbb{S}^{m} \times \mathbb{S}^{m} \\
d_{\mathcal{N}}(f(y), f(x)) \geq \delta}} \frac{\left(d_{\mathcal{N}}(f(y), f(x))-\delta\right)^{p}}{|y-x|^{2 m}} \mathrm{~d} y \mathrm{~d} x .
\end{aligned}
$$

We conclude by combining (5.7) and (5.8) with $C=(m+2) C_{1}$.

Theorem 5.4 (Free homotopy decompositions controlled by a scaled truncated Sobolev energy). Let $m \in$ $\mathbb{N}_{\star}$ and $\mathcal{N}$ be a compact Riemannian manifold. There are constants $\varepsilon_{0}>0$ and $C>0$ such that for every $\lambda>0$, there exists a finite set $\mathscr{F}^{\lambda} \subset \mathscr{C}\left(\mathbb{S}^{m}, \mathcal{N}\right)$ such that if $0<\varepsilon<\varepsilon_{0}$, any map $f \in \mathscr{C}\left(\mathbb{S}^{m}, \mathcal{N}\right)$ satisfying

$$
\varepsilon^{m-1} \iint_{\mathbb{S}^{m} \times \mathbb{S}^{m}} \frac{\left(d_{\mathcal{N}}(f(y), f(x))-\varepsilon\right)_{+}}{|y-x|^{2 m}} \mathrm{~d} y \mathrm{~d} x \leq \lambda,
$$

has a free homotopy decomposition into $f_{1}, \ldots, f_{k} \in \mathscr{F}^{\lambda}$ with $k \leq C \lambda$. 
Proof. This follows from theorem 4.6 and proposition 5.2.

\subsection{Comparison between fractional truncated energies}

In passing form theorem 4.6 to theorem 1.3 we relied on (4.8), which is not optimal when $\varepsilon$ is small and $f$ is the identity mapping (see (5.1)). In this section, we derive estimates that compare different gap integrals with optimal scaling on a convex subset $\Omega$ of the Euclidean space $\mathbb{R}^{m}$.

Proposition 5.5 (Comparison between truncated fractional energies). For every $m \geq 2, p \in[0, m), q \in$ $[0,+\infty)$ and $\eta \in(0,1)$, there exists a constant $C>0$ such that for every convex set $\Omega \subset \mathbb{R}^{m}$, for every map $f: \Omega \rightarrow \mathcal{N}$ and for every $\varepsilon>0$, one has

$$
\iint_{\substack{(x, y) \in \Omega \times \Omega \\
d_{\mathcal{N}}(f(y), f(x)) \geq \varepsilon}} \frac{\left(d_{\mathcal{N}}(f(y), f(x))-\varepsilon\right)^{p}}{|y-x|^{2 m}} \mathrm{~d} y \mathrm{~d} x \leq C \varepsilon^{p-q} \iint_{\begin{array}{r}
(x, y) \in \Omega \times \Omega \\
d_{\mathcal{N}}(f(y), f(x)) \geq \eta \varepsilon
\end{array}} \frac{\left(d_{\mathcal{N}}(f(y), f(x))-\eta \varepsilon\right)^{q}}{|y-x|^{2 m}} \mathrm{~d} y \mathrm{~d} x .
$$

In view of the asymptotics (5.1) on the integrals when $f$ is the identity, the scaling of the estimate in proposition 5.5 is optimal and the estimate of proposition 5.5 fails when $p \geq m$ and $p>q$.

When $p<q$, the estimate follows from the elementary inequality: for $t \geq \varepsilon$,

$$
(t-\varepsilon)^{p} \leq \frac{(t-\eta \varepsilon)^{q}}{(1-\eta)^{q-p} \varepsilon^{q-p}}
$$

the interest of the estimate lies essentially thus in the case $q<p<m$. The proof of theorem 5.8 , will be relying only on the case $q=0$ and $p=1$.

The proof of proposition 5.5 relies on proposition 5.1 and the next lemma 5.6.

Lemma 5.6 (Integral estimate of truncated powers). For every $p, q \in[0,+\infty)$ and $\eta \in(0,1)$, there exists a constant $C>0$ such that for every $t, s \in[0,+\infty)$ such that $t \geq s$,

$$
(t-s)^{p} \leq C \int_{\eta s}^{t} \frac{(t-r)^{q}}{r^{1+q-p}} \mathrm{~d} r
$$

If $p=q+1$, lemma 5.6 has a direct proof with $\eta=1$ : indeed, for every $t, s \in \mathbb{R}$, one has

$$
(t-s)^{p}=p \int_{s}^{t} \frac{(t-r)^{q}}{r^{1+q-p}} \mathrm{~d} r .
$$

Proof of lemma 5.6. If we set $t=\tau s$, with $\tau \geq 1$, we have

$$
(t-s)^{p}=s^{p}(\tau-1)^{p}
$$

and, under the change of variables $r=s \rho$,

$$
\int_{\eta s}^{t} \frac{(t-r)^{q}}{r^{1+q-p}} \mathrm{~d} r=s^{p} \int_{\eta}^{\tau} \frac{(\tau-\rho)^{q}}{\rho^{1+q-p}} \mathrm{~d} \rho .
$$

We observe that the function $g:[1,+\infty) \rightarrow \mathbb{R}$ defined for each $\tau \in[1,+\infty)$ by

$$
g(\tau) \triangleq \int_{\eta}^{\tau} \frac{(\tau-\rho)^{q}}{\rho^{1+q-p}} \mathrm{~d} \rho,
$$

is continuous and positive and that by the change of variable $\rho=\tau \sigma$,

$$
\lim _{\tau \rightarrow+\infty} \frac{g(\tau)}{\tau^{p}}=\lim _{\tau \rightarrow+\infty} \int_{\eta / \tau}^{1} \frac{(1-\sigma)^{q}}{\sigma^{1+q-p}} \mathrm{~d} \sigma=\int_{0}^{1} \frac{(1-\sigma)^{q}}{\sigma^{1+q-p}} \mathrm{~d} \sigma>0 .
$$

Hence we have $(\tau-1)^{p} \leq C g(\tau)$ for each $\tau \in[1,+\infty)$ and the conclusion follows by (5.9) and (5.10). 
Proof of proposition 5.5. We first observe that by lemma 5.6 applied at each $x, y \in \Omega$ with $t=d_{\mathcal{N}}(f(y), f(x))$ and $s=\varepsilon$, we have

$$
\iint_{\substack{(x, y) \in \Omega \times \Omega \\ d_{\mathcal{N}}(f(y), f(x)) \geq \varepsilon}} \frac{\left(d_{\mathcal{N}}(f(y), f(x))-\varepsilon\right)^{p}}{|y-x|^{2 m}} \mathrm{~d} y \mathrm{~d} x \leq C_{1} \int_{\substack{\eta \varepsilon \\(x, y) \in \Omega \times \Omega) \geq r \\ d_{\mathcal{N}}(f(y), f(x)) \geq r}}^{+\infty} \iint \frac{\left(d_{\mathcal{N}}(f(y), f(x))-r\right)^{q}}{r^{1+q-p}|y-x|^{2 m}} \mathrm{~d} y \mathrm{~d} x \mathrm{~d} r .
$$

Since the set $\Omega \subseteq \mathbb{R}^{m}$ is convex, by proposition 5.1, we have for every $r \in(0,+\infty)$,

$$
\iint_{\substack{(x, y) \in \Omega \times \Omega \\
d_{\mathcal{N}}(f(y), f(x)) \geq r}} \frac{\left(d_{\mathcal{N}}(f(y), f(x))-r\right)^{q}}{|y-x|^{2 m}} \mathrm{~d} y \mathrm{~d} x \leq C_{2}\left(\frac{\varepsilon}{r}\right)^{(m-1)-(q-1)_{+}} \iint_{\begin{array}{c}
(x, y) \in \Omega \times \Omega \\
d_{\mathcal{N}}(f(y), f(x)) \geq \eta \varepsilon
\end{array}} \frac{\left(d_{\mathcal{N}}(f(y), f(x))-\eta \varepsilon\right)^{q}}{|y-x|^{2 m}} \mathrm{~d} y \mathrm{~d} x .
$$

By combining (5.11) and (5.12), we deduce equation

$$
\iint_{\substack{(x, y) \in \Omega \times \Omega \\ d_{\mathcal{N}}(f(y), f(x)) \geq \varepsilon}} \frac{\left(d_{\mathcal{N}}(f(y), f(x))-\varepsilon\right)^{p}}{|y-x|^{2 m}} \mathrm{~d} y \mathrm{~d} x \leq C_{3} \int_{\eta \varepsilon}^{+\infty} \frac{\varepsilon^{(m-1)-(q-1)_{+}}}{r^{m+1-(1-q)_{+}-p}} \mathrm{~d} r \underset{\substack{(x, y) \in \Omega \times \Omega \\ d_{\mathcal{N}}(f(y), f(x)) \geq \eta \varepsilon}}{\iint_{\mathcal{N}}} \frac{\left(d_{\mathcal{N}}(f(y), f(x))-\eta \varepsilon\right)^{p}}{|y-x|^{2 m}} \mathrm{~d} y \mathrm{~d} x .
$$

If $p<m-(1-q)_{+}$, then

$$
\begin{aligned}
\int_{\eta \varepsilon}^{+\infty} \frac{\varepsilon^{(m-1)-(q-1)_{+}}}{r^{m+1-(1-q)_{+}-p}} \mathrm{~d} r & =\frac{\varepsilon^{(m-1)-(q-1)_{+}}}{\left(m-(1-q)_{+}-p\right)(\eta \varepsilon)^{m-(1-q)_{+}-p}} \\
& =\frac{\varepsilon^{p-q}}{\left(m-(1-q)_{+}-p\right) \eta^{m-(1-q)_{+}-p}}
\end{aligned}
$$

and the estimate is satisfied.

In order to cover the case $m-(1-q)_{+} \leq p<m$, we observe that since $m \geq 2$ and $q \geq 0$, the estimate holds for every $p \in[0,1)$. By iterating a second time the estimate, we obtain the estimate for each $p \in[0, m)$.

The proof shows that when $m=1$, the estimate of proposition 5.5 holds if $p<\min (1, q)$, in which case the estimate is in fact elementary.

The estimate of proposition 5.5 also holds when the domain is a sphere $\mathbb{S}^{m}$.

Proposition 5.7 (Comparison of truncated fractional energies on the sphere). For every $m \geq 2, p \in[0, m$ ), $q \in[0,+\infty)$ and $\eta \in(0,1)$, there exists a constant $C>0$ such that for every map $f: \mathbb{S}^{m} \rightarrow \mathcal{N}$ and for every $\varepsilon>0$, one has

$$
\iint_{\substack{(x, y) \in \mathbb{S}^{m} \times \mathbb{S}^{m} \\
d_{\mathcal{N}}(f(y), f(x)) \geq \varepsilon}} \frac{\left(d_{\mathcal{N}}(f(y), f(x))-\varepsilon\right)^{p}}{|y-x|^{2 m}} \mathrm{~d} y \mathrm{~d} x \leq C \varepsilon^{p-q} \iint_{\begin{array}{c}
(x, y) \in \mathbb{S}^{m} \times \mathbb{S}^{m} \\
d_{\mathcal{N}}(f(y), f(x)) \geq \eta \varepsilon
\end{array}} \frac{\left(d_{\mathcal{N}}(f(y), f(x))-\eta \varepsilon\right)^{q}}{|y-x|^{2 m}} \mathrm{~d} y \mathrm{~d} x .
$$

Proof. The proof follows the lines of the proof of proposition 5.2, relying on the covering given by lemma 5.3 and the estimate on a convex set of proposition 5.5.

We conclude this section with a scaled version of theorem 1.3.

Theorem 5.8 (Free homotopy decompositions controlled by a scaled gap potential). Let $m \in \mathbb{N} \backslash\{0,1\}$ and $\mathcal{N}$ be a compact Riemannian manifold. There are constants $\varepsilon_{0}>0$ and $C>0$, such that for every $\lambda>0$, there exists a finite set $\mathscr{F}^{\lambda} \subset \mathscr{C}\left(\mathbb{S}^{m}, \mathcal{N}\right)$ such that if $0<\varepsilon<\varepsilon_{0}$, any map $f \in \mathscr{C}\left(\mathbb{S}^{m}, \mathcal{N}\right)$ satisfying

$$
\iint_{\substack{(x, y) \in \mathbb{S}^{m} \times \mathbb{S}^{m} \\ d_{\mathfrak{N}}(f(y), f(x))>\varepsilon}} \frac{\varepsilon^{m}}{|y-x|^{2 m}} \mathrm{~d} y \mathrm{~d} x \leq \lambda
$$

has a free homotopy decomposition into $f_{1}, \ldots, f_{k} \in \mathscr{F}^{\lambda}$ with $k \leq C \lambda$. 
We do not know whether theorem 5.8 holds when $m=2$ (see open problem 1 below).

Proof of theorem 5.8. Since $m \geq 2$, by proposition 5.7, we have for every $f: \mathbb{S}^{m} \rightarrow \mathcal{N}$ and every $\varepsilon>0$,

$$
\varepsilon^{m-1} \iint_{\mathbb{S}^{m} \times \mathbb{S}^{m}} \frac{\left(d_{\mathcal{N}}(f(y), f(x))-\varepsilon\right)_{+}}{|y-x|^{2 m}} \mathrm{~d} y \mathrm{~d} x \leq C_{1} \varepsilon^{m} \iint_{\begin{array}{c}
(x, y) \in \mathbb{S}^{m} \times \mathbb{S}^{m} \\
d_{\mathcal{N}}(f(y), f(x))>\varepsilon
\end{array}} \frac{1}{|y-x|^{2 m}} \mathrm{~d} y \mathrm{~d} x ;
$$

the conclusion then follows from theorem 5.4 .

\section{Estimates of the Hurewicz homomorphism on the sphere}

The Hurewicz homomorphism is a homotopy invariant of maps that describes how a mapping from $\mathbb{S}^{m}$ to $\mathcal{N}$ acts on the cohomology of $\mathcal{N}$. For a smooth map $f \in \mathscr{C}^{1}\left(\mathbb{S}^{m}, \mathcal{N}\right)$ and for a closed differential form $\omega \in \mathscr{C}^{1}\left(\bigwedge^{m} \mathcal{N}\right)$, that is, a form such that $d \omega=0$, we define

$$
\langle\operatorname{Hur}(f), \omega\rangle \triangleq \int_{\mathbb{S}^{m}} f^{\star} \omega,
$$

where $f^{\star} \omega \in C\left(\bigwedge^{m} \mathbb{S}^{m}\right)$ denotes the pull-back by the map $f$ of the form $\omega$. We note that Hur acts trivially on exact forms: if $\omega=d \eta$ with $\eta \in \mathscr{C}^{2}\left(\bigwedge^{m-1} \mathcal{N}\right)$, then by the Stokes-Cartan theorem

$$
\langle\operatorname{Hur}(f), \omega\rangle=\int_{\mathbb{S}^{m}} f^{\star}(d \eta)=\int_{\mathbb{S}^{m}} d\left(f^{\star} \eta\right)=0,
$$

and thus by quotienting by the space of exact forms $d\left(\mathscr{C}^{2}\left(\bigwedge^{m-1} \mathcal{N}\right)\right)$, Hur $(f)$ induces a linear map on the de Rham cohomology $H_{\mathrm{dR}}^{m}(\mathcal{N})$, which by de Rham's theorem, defines then an element in the singular homology group $\operatorname{Hur}(f) \in H_{m}(\mathcal{N}, \mathbb{R}) \simeq H_{m}(\mathcal{N}, \mathbb{Z}) \otimes \mathbb{R}$. This map $\operatorname{Hur}(f)$ does not depend on the homotopy of the original $\operatorname{map} f:$ if $H \in \mathscr{C}^{2}\left(\mathbb{S}^{m} \times[0,1]\right) \rightarrow \mathcal{N}$ is a homotopy, then

$$
\int_{\mathbb{S}^{m}} H(\cdot, 1)^{\star} \omega-\int_{\mathbb{S}^{m}} H(\cdot, 0)^{\star} \omega=(-1)^{m} \int_{\mathbb{S}^{m} \times[0,1]} d\left(H^{\star} \omega\right)=0 .
$$

In particular, Hur induces a map from the homotopy group $\pi_{m}(\mathcal{N})$ to the homology group $H_{m}(\mathcal{N}, \mathbb{R})$. The Hurewicz homomorphism generalizes the degree of maps into the sphere: if $\mathcal{N}=\mathbb{S}^{n}$, we have $\left\langle\operatorname{Hur}(f), \omega_{\mathbb{S}^{n}}\right\rangle=$ $\operatorname{deg}(f)$; it extends more generally the degree of maps when $\operatorname{dim} \mathcal{N}=m[19 ; 22, \S 8]$.

Since the Hurewicz homomorphism is invariant under homotopies, it is well-defined for maps of vanishing mean oscillation. Moreover, by standard approximation, the formula (6.1) is still valid whenever $f \in W^{1, m}\left(\mathbb{S}^{m}, \mathcal{N}\right)$ (see [11, (19)]).

The estimate (1.2) can be generalized immediately to the Hurewicz homomorphism: if $f \in W^{1, m}\left(\mathbb{S}^{m}, \mathcal{N}\right)$, then

$$
|\operatorname{Hur}(f)| \leq C \int_{\mathbb{S}^{m}}|D f|^{m} .
$$

Indeed, this follows from the definition of Hur in (6.1) and the fact that $\left|f^{\star}(\omega)\right| \leq|\omega||D f|^{m}$ almost everywhere on $\mathbb{S}^{m}$. When $\mathcal{N}=\mathbb{S}^{m}$, then (6.2) is equivalent to the degree estimate (1.2).

Theorem 6.1 (Estimate of Hurewicz homomorphism by a truncated fractional energy). Let $m \in \mathbb{N}_{\star}$ and $\mathcal{N}$ be a compact Riemannian manifold. If $\varepsilon>0$ is small enough, then there exists a constant $C>0$ such that if $f \in \mathscr{C}\left(\mathbb{S}^{m}, \mathcal{N}\right)$, then

$$
|\operatorname{Hur}(f)| \leq C \iint_{\mathbb{S}^{m} \times \mathbb{S}^{m}} \frac{\left(d_{\mathcal{N}}(f(y), f(x))-\varepsilon\right)_{+}}{|y-x|^{2 m}} \mathrm{~d} y \mathrm{~d} x .
$$

When $m \geq 2$ (6.2) can be deduced from theorem 6.1 since the right-hand side in theorem 6.1 can always be controlled by the right-hand side in (6.2) [35, lemma 2]. When $m=1$, there is no such estimate [35, proposition 3], but it might be possible to refine existing $\Gamma$-convergence results [40, theorem 2] in order to deduce (6.2) from theorem 6.1. 
Proof of theorem 6.1. Since $\mathcal{N}$ is a compact manifold embedded into $\mathbb{R}^{v}$, there exists an open set $U \subset \mathbb{R}^{v}$ such that $\mathcal{N} \subset U$ and a smooth retraction $\Pi \in \mathscr{C}^{\infty}(U, \mathcal{N})$. We also consider a smooth map $\eta \in \mathscr{C}_{c}^{\infty}(U, \mathbb{R})$ such that $\eta(y)=1$ if $y \in \mathcal{N}$. Given $f \in \mathscr{C}\left(\mathbb{S}^{m}, \mathcal{N}\right)$, we let $F \in \mathscr{C}^{\infty}\left(\mathbb{B}^{m+1}, \mathbb{R}^{v}\right)$ be given by proposition 4.1 and we compute by the Stokes-Cartan formula

$$
\begin{aligned}
\langle\operatorname{Hur}(f), \omega\rangle=\int_{\mathbb{S}^{m}} f^{\star} \omega & =\int_{\mathbb{S}^{m}} F^{\star}\left(\eta \wedge \Pi^{\star} \omega\right) \\
& =\int_{\mathbb{B}^{m+1}} d\left(F^{\star}\left(\eta \wedge \Pi^{\star} \omega\right)\right)=\int_{\mathbb{B}^{m+1}} F^{\star}\left(d \eta \wedge \Pi^{\star} \omega\right),
\end{aligned}
$$

since $d\left(\Pi^{\star} \omega\right)=\Pi_{\#}(d \omega)=0$. Hence we have, by the estimates given by proposition 4.1

$$
\begin{aligned}
|\langle\operatorname{Hur}(f), \omega\rangle| & \leq \mu_{\mathbb{H}^{m+1}}\left(\left\{x \in \mathbb{H}^{m+1} \mid \operatorname{dist}(F(x), \mathcal{N}) \geq \delta\right\}\right)\|D F\|_{L^{\infty}\left(\mathbb{H}^{m+1}\right)}^{m+1}\|\omega\|_{L^{\infty}} \\
& \leq C_{1}\|\omega\|_{L^{\infty}} \iint_{\mathbb{S}^{m} \times \mathbb{S}^{m}} \frac{\left(d_{\mathcal{N}}(f(y), f(x))-\varepsilon\right)_{+}}{|y-x|^{2 m}} \mathrm{~d} y \mathrm{~d} x .
\end{aligned}
$$

We also have an estimate of the Hurewicz homomorphism with optimal scaling when $m \geq 2$.

Theorem 6.2 (Estimate of the Hurewicz homomorphism by a scaled gap potential). If $m \geq 2$ and $\mathcal{N}$ is a compact Riemannian manifold, then there exists constants $C>0$ and $\varepsilon_{0}>0$ such that if $\varepsilon \in\left(0, \varepsilon_{0}\right)$ and $f \in \mathscr{C}\left(\mathbb{S}^{m}, \mathcal{N}\right)$, then

$$
|\operatorname{Hur}(f)| \leq C \quad \iint_{\substack{x, y \in \mathbb{S}^{m} \\ d_{\mathcal{N}}(f(y), f(x)) \geq \varepsilon}} \frac{\varepsilon^{m}}{|y-x|^{2 m}} \mathrm{~d} y \mathrm{~d} x .
$$

Proof. This follows from theorem 6.1 in view of proposition 5.2 and proposition 5.7.

When $\mathcal{N}=\mathbb{S}^{m}$ we recover the estimate on the degree of NGUYÊN Hoài-Minh [43]; the latter estimate was obtained through the John-Nirenberg estimate and seems different from our direct approach. When $m=1$, the question whether theorem 6.2 holds is an open problem (open problem 1).

\section{Homotopy estimates on a compact manifold}

\subsection{Free homotopy decompositions upon a mapping}

We consider the problem of controlling the homotopy classes of maps from a general compact manifold $\mathcal{M}$ to another compact manifold $\mathcal{N}$. The notion of free homotopy decomposition (definition 1.1) generalizes into the free homotopy decomposition upon a mapping. Since the circle $\mathbb{S}^{1}$ is, up to a conformal transformation, the only connected compact one-dimensional Riemannian manifold, we assume throughout this section that $\operatorname{dim}(\mathcal{M})=m \geq 2$.

Definition 7.1 (Free homotopy decomposition upon a mapping). Let $\mathcal{N}$ and $\mathcal{N}$ be connected compact Riemannian manifolds and let $m=\operatorname{dim} \mathcal{N}$. A map $f \in \mathscr{C}(\mathcal{M}, \mathcal{N})$ has a free homotopy decomposition into the maps $f_{1}, \ldots, f_{k} \in \mathscr{C}\left(\mathbb{S}^{m}, \mathcal{N}\right)$ upon the map $f_{0} \in \mathscr{C}(\mathcal{M}, \mathcal{N})$ whenever there exist maps $g, g_{0} \in \mathscr{C}(\mathcal{N}, \mathcal{N})$ and nondegenerate topologically trivial balls $B_{\rho_{0}}\left(a_{0}\right), \ldots, B_{\rho_{\ell}}\left(a_{k}\right)$, such that $g$ is homotopic to $f$, $g_{0}$ is homotopic to $f_{0}, g=g_{0}$ on $\mathcal{M} \backslash B_{\rho_{0}}\left(a_{0}\right), g_{0}$ is constant on $\bar{B}_{\rho_{0}}\left(a_{0}\right)$, and for every $i \in\{1, \ldots, k\}, \bar{B}_{\rho_{i}}\left(a_{i}\right) \subset B_{\rho_{0}}\left(a_{0}\right)$ and $\left.g\right|_{\bar{B}_{\rho_{i}}\left(a_{i}\right)}$ is homotopic to $f_{i}$ on $\mathbb{S}_{m} \simeq \bar{B}_{\rho_{i}}\left(a_{i}\right) / \partial B_{\rho_{i}}\left(a_{i}\right)$.

Remark 7.2. When $\mathcal{M}=\mathbb{S}^{m}$, a map $f \in \mathscr{C}\left(\mathbb{S}^{m}, \mathcal{N}\right)$ has a free homotopy decomposition into $f_{1}, \ldots, f_{k} \in$ $\mathscr{C}\left(\mathbb{S}^{m}, \mathcal{N}\right)$ in the sense of definition 1.1 if and only if $f$ has a free homotopy decomposition into $f_{1}, \ldots, f_{k} \in$ $\mathscr{C}\left(\mathbb{S}^{m}, \mathcal{N}\right)$ upon a constant $\operatorname{map} f_{0} \in \mathscr{C}\left(\mathbb{S}^{m}, \mathcal{N}\right)$ in the sense of definition 7.1. 
The next proposition describes free homotopy decompositions upon a mapping on a general manifold through free homotopy decompositions on the sphere.

Proposition 7.3. Let $f_{0} \in \mathscr{C}(\mathcal{M}, \mathcal{N})$ and $f_{1}, \ldots, f_{k} \in\left(\mathbb{S}^{m}, \mathcal{N}\right)$. Assume that $f_{0}$ is constant on some nondegenerate topologically trivial ball $B_{\rho}(a) \subset \mathcal{M}$. The map $f \in \mathscr{C}(\mathcal{M}, \mathcal{N})$ has a free homotopy decomposition into $f_{1}, \ldots, f_{k}$ upon $f_{0}$ if and only there exists a map $h \in \mathscr{C}(\mathcal{N}, \mathcal{N})$ such that $h$ is homotopic to $f, h=f_{0}$ on $\mathcal{N} \backslash B_{\rho}(a)$ and $\left.h\right|_{\bar{B}_{\rho}(a)}$ has a free homotopy decomposition into $f_{1}, \ldots, f_{k}$ under the identification $\mathbb{S}^{m} \simeq \bar{B}_{\rho}(a) / \partial B_{\rho}(a)$.

Since the definition of free homotopy decomposition upon a mapping (definition 7.1) is invariant under homotopies, the condition that the $\operatorname{map} f_{0}$ is constant on some nondegenerate topologically trivial ball can always be satisfied. Free decompositions upon a given mapping on a manifold are thus not more complex than a collection of homotopy classes of maps on a sphere relative to some point. The free homotopy decompositions into given maps upon a given map can be precisely identified and enumerated by obstruction cohomology classes with local groups $[2, \S 4.2 ; 28$, Chapter VI].

Proof of proposition 7.3. If the map $f$ is homotopic to $h$, it follows directly from the definition of free decompositions definition 1.1 and definition 7.1 that $f$ has a free homotopy decomposition into $f_{1}, \ldots, f_{k}$ upon $f_{0}$.

Conversely, let us assume that $f \in \mathscr{C}(\mathcal{M}, \mathcal{N})$ has a free homotopy decomposition into $f_{1}, \ldots, f_{k}$ upon $f_{0}$, and let $g, g_{0} \in \mathscr{C}(\mathcal{M}, \mathcal{N})$ and the balls $B_{\rho_{0}}\left(a_{0}\right), \ldots, B_{\rho_{k}}\left(a_{k}\right)$ be given by definition 7.1. Without loss of generality, we can assume that $B_{\rho_{0}}\left(a_{0}\right)=B_{\rho / 2}(a)$. Since the maps $f_{0}$ and $g_{0}$ are homotopic, there exists a homotopy $H \in \mathscr{C}(\mathcal{M} \times[0,1], \mathcal{N})$ such that $H(\cdot, 0)=g_{0}$ and $H(\cdot, 1)=f_{0}$ on $\mathcal{M}$ and such that for every $t \in[0,1]$, $H(\cdot, t)$ is constant on $B_{\rho}(a)$. This implies in turn the existence of a homotopy $\tilde{H} \in \mathscr{C}(\mathcal{N} \times[0,1], \mathcal{N})$ such that $\tilde{H}(\cdot, 0)=g$ on $\mathcal{M}, \tilde{H}(\cdot, 1)=f_{0}$ on $\mathcal{M} \backslash B_{\rho}(a)$, for every $t \in[0,1], \tilde{H}(\cdot, t)$ is radial on $B_{\rho}(a) \backslash B_{\rho / 2}(a)$ and $\tilde{H}(\cdot, t)=g$ on $B_{\rho / 2}(a)$. We set $h \triangleq \tilde{H}(\cdot, 1)$ and we conclude by the observation that $\left.h\right|_{B_{\rho}(a)}$ has a free homotopy decomposition into $f_{1}, \ldots, f_{k}$ under the identification $\mathbb{S}^{m} \simeq B_{\rho}(a) / \partial B_{\rho}(a)$ and that by transitivity of homotopies $h$ is homotopic to $f$.

As a consequence we of proposition 7.3, we prove a counterpart of proposition 2.3 for free homotopy decompositions upon a mapping asserting the finiteness of homotopy classes sharing a given free homotopy decomposition.

Proposition 7.4. If $m \geq 2$ and every orbit of the action of $\pi_{1}(\mathcal{N})$ on $\pi_{m}(\mathcal{N})$ is finite, then for every $f_{0} \in \mathscr{C}(\mathcal{M}, \mathcal{N})$ and $f_{1}, \ldots, f_{k} \in \mathscr{C}\left(\mathbb{S}^{m}, \mathcal{N}\right)$, there exists a finite set $\mathscr{G} \subset \mathscr{C}\left(\mathbb{S}^{m}, \mathcal{N}\right)$ such that every map $f \in \mathscr{C}\left(\mathbb{S}^{m}, \mathcal{N}\right)$ that has a free homotopy decomposition into $f_{1}, \ldots, f_{k}$ upon $f_{0}$ is homotopic to some $g \in \mathscr{G}$.

Proof. We assume up to a homotopy and without loss of generality that $f_{0}=b \in \mathcal{N}$ on a trivial ball $B_{\rho}(a) \subset \mathcal{M}$. We consider $y_{1}, \ldots, y_{k} \in \pi_{m}(\mathcal{N}, b)$ respectively homotopic to $f_{1}, \ldots, f_{k}$ and we set

$$
\Gamma \triangleq\left\{\beta_{1} \cdot y_{1}+\cdots+\beta_{k} \cdot y_{k} \mid \beta_{1}, \ldots, \beta_{k} \in \pi_{1}(\mathcal{N}, b)\right\} .
$$

Since by assumption for every $i \in\{1, \ldots, k\}$ the set $\left\{\beta_{i} \cdot y_{i} \mid \beta_{i} \in \pi_{1}(\mathcal{N}, b)\right\}$ is finite, the set $\Gamma$ is finite and we can construct $\tilde{\mathscr{G}} \subset \mathscr{C}\left(B_{\rho}(a), \mathcal{N}\right)$ as a finite set of mappings taking the constant value $b$ on $\partial B_{\rho}(a)$ and such that under the identification $\mathbb{S}^{m} \simeq B_{\rho}(a) / \partial B_{\rho}(a)$, every element of $\Gamma$ is homotopic to some map in $\tilde{\mathscr{G}}$. We define now

$$
\mathscr{G} \triangleq\left\{g \in \mathscr{C}(\mathcal{M}, \mathcal{N}) \mid g=f_{0} \text { in } \mathcal{M} \backslash B_{\rho}(a) \text { and }\left.g\right|_{B_{\rho}(a)} \in \tilde{\mathscr{G}}\right\} .
$$

By proposition 7.3, and proposition 2.2 any map $f \in \mathscr{C}(\mathcal{M}, \mathcal{N})$ that has a free homotopy decomposition into $f_{1}, \ldots, f_{k}$ upon $f_{0}$ is homotopic to some $g \in \mathscr{G}$.

\subsection{Estimates of free homotopy decompositions}

The counterpart of theorem 1.3 when the domain is a general compact Riemannian manifold manifold $\mathcal{M}$ is the following 
Theorem 7.5 (Free decomposition estimate by a gap potential). Let $\mathcal{N}$ and $\mathcal{N}$ be connected compact Riemannian manifolds. If $\varepsilon>0$ is small enough, then there is a constant $C>0$ such that for every $\lambda>0$, there exist finite sets $\mathscr{F}^{\lambda} \subset \mathscr{C}\left(\mathbb{S}^{m}, \mathcal{N}\right)$ and $\mathcal{F}_{0}^{\lambda} \subset \mathscr{C}(\mathcal{M}, \mathcal{N})$ such that any $f \in \mathscr{C}(\mathcal{M}, \mathcal{N})$ satisfying

$$
\iint_{\substack{(x, y) \in \mathcal{M} \times \mathcal{M} \\ d_{\mathcal{N}}(f(y), f(x))>\varepsilon}} \frac{1}{d_{\mathcal{M}}(y, x)^{2 m}} \mathrm{~d} x \mathrm{~d} y \leq \lambda
$$

has a free homotopy decomposition into $f_{1}, \ldots, f_{k} \in \mathscr{F}^{\lambda}$ upon $f_{0} \in \mathscr{F}_{0}^{\lambda}$, with $k \leq C \lambda$.

In view of remark 7.2, theorem 1.3 corresponds to the particular case $\mathcal{M}=\mathbb{S}^{m}$ in theorem 7.5.

In order to follow in the proof of theorem 7.5 the same strategy as in the proof of theorem 1.3, we construct a Riemannian manifold that is the counterpart of the hyperbolic space $\mathbb{H}^{m+1}$ for $\mathbb{S}^{m}$. We define the manifold $\mathcal{M}^{*} \triangleq \mathcal{M} \times(0,+\infty)$ and we endow it with a metric $g^{\mathcal{M}^{*}}$ defined as a quadratic form for each point $(x, t) \in \mathcal{M}^{*}$ and each tangent vector $(v, w) \in T_{(x, t)} \mathcal{M}^{\star} \simeq T_{x} \mathcal{M} \times \mathbb{R}$ by

$$
g_{(x, t)}^{\mathcal{M}^{*}}(v, w) \triangleq \frac{g_{x}(v)+w^{2}}{t^{2}},
$$

where $g$ is the metric of the original manifold $\mathcal{M}$. When $\mathcal{M}=\mathbb{R}^{m}$, the manifold $\mathcal{M}^{*}$ is the Poincare half-space model of the hyperbolic space. The formula (7.1) shows that the manifold $\mathcal{M}^{*}$ is conformally equivalent to the Riemannian Cartesian product $\mathcal{M} \times(0,+\infty)$.

Remark 7.6. The manifold $\mathcal{M}^{*}$ is in fact a warped product: if $\mathcal{M}^{\diamond} \triangleq \mathcal{M} \times \mathbb{R}$ is endowed with the metric $g^{\mathcal{M}}$ defined as a quadratic form for each $(x, t) \in \mathcal{M}^{*}$ and $(v, w) \in T_{(x, t)} \mathcal{M}^{*} \simeq T_{x} \mathcal{M} \times \mathbb{R}$ by $g_{(x, t)}^{\mathcal{N} \mathcal{K}^{\diamond}}(v, w) \triangleq e^{-2 s} g_{x}(v)+w^{2}$, the mapping $(x, s) \in \mathcal{M}^{\diamond} \triangleq \mathcal{M} \times \mathbb{R} \mapsto\left(x, e^{s}\right) \in \mathcal{M}^{*}$ is an isometry. (In fact $\mathcal{M}^{*}$ is the conformal representation of the warped product $\mathcal{M}^{\diamond}[46, \S 4.3]$.)

\subsubsection{Extension}

The first tool that we need is a controlled extension to $\mathcal{M}$ corresponding to proposition 4.1.

Proposition 7.7 (Extension to $\left.\mathcal{M}^{\star}\right)$. Let $\mathcal{M}$ be compact Riemannian manifold. There exists a constant $C>0$ such that for every $f \in \mathscr{C}\left(\mathbb{S}^{m}, \mathbb{R}^{v}\right)$, there exists a function $F \in \mathscr{C}\left(\mathcal{M} \times[0,+\infty), \mathbb{R}^{v}\right) \cap \mathscr{C}^{\infty}\left(\mathcal{M} \times(0,+\infty), \mathbb{R}^{v}\right)$ such that

(i) $F(\cdot, 0)=f$ on $\mathcal{M}$,

(ii) for every $z \in \mathcal{M}^{*} \simeq \mathcal{M} \times(0,+\infty)$,

$$
|D F(z)|_{\mathcal{M}^{*}} \leq \underset{\mathbb{S}^{m}}{\operatorname{oos} f}
$$

(iii) if $\delta>\varepsilon$, then

$$
\mu_{\mathcal{M}^{*}}\left(\left\{x \in \mathcal{M}^{*} \mid \operatorname{dist}\left(F(x), f\left(\mathbb{S}^{m}\right)\right) \geq \delta\right\}\right) \leq \frac{C}{\delta-\varepsilon} \iint_{\mathcal{M} \times \mathcal{M}} \frac{(|f(y)-f(x)|-\varepsilon)_{+}}{d_{\mathcal{M}}(y, x)^{2 m}} \mathrm{~d} y \mathrm{~d} x .
$$

The proof of proposition 4.1 relied on the hyperharmonic extension defined in (4.4). In order to define a similar extension, we introduce a suitable integral kernel.

Lemma 7.8 (Approximation of the identity on $\mathcal{M}$ along $\mathcal{M}^{\star}$ ). Let $\mathcal{M}$ be an m-dimensional compact Riemannian manifold. There exists a function $\Phi \in \mathscr{C}^{\infty}\left(\mathcal{M}^{*} \times \mathcal{M},[0,+\infty)\right)$ and a constant $C>0$ such that

(i) for every $(x, t) \in \mathcal{M}^{*}=\mathcal{M} \times(0,+\infty)$,

$$
\int_{\mathcal{M}} \Phi(x, t, \cdot)=1
$$


(ii) for every $(x, t, y) \in \mathcal{M}^{\star} \times \mathcal{M}=\mathcal{M} \times(0,+\infty) \times \mathcal{M}$,

$$
\Phi(x, t, y) \leq \frac{C t^{m}}{d_{\mathcal{M}}(y, x)^{2 m}} .
$$

(iii) if we set for every $(x, t, y) \in \mathcal{M}^{*} \times \mathcal{M}=\mathcal{M} \times(0,+\infty) \times \mathcal{M}, \Phi_{y}(x, t)=\Phi(x, t, y)$, then for every $(x, t) \in \mathcal{M}^{*}=$ $\mathcal{M} \times(0,+\infty)$,

$$
\int_{\mathcal{M}}\left|D \Phi_{y}(x, t)\right|_{\mathcal{M}^{*}} \mathrm{~d} y \leq C .
$$

Proof. We choose a function $\varphi: \mathscr{C}^{\infty}(\mathbb{R},[0,+\infty))$ such that $\varphi(0)>0$, and $\varphi=0$ on $\mathbb{R} \backslash(-1,1)$, and

$$
\int_{\mathbb{R}^{m}} \varphi\left(|z|^{2}\right) \mathrm{d} z=1
$$

Since the manifold $\mathcal{M}$ is compact, there exists $\delta>0$ such that if $E \triangleq\left\{(x, y) \in \mathcal{M} \times \mathcal{M} \mid d_{\mathcal{M}}(x, y)<\delta\right\}$ the function $(x, y) \in E \mapsto d_{\mathcal{M}}(x, y)^{2}$ is smooth. We fix a function $\eta \in \mathscr{C}^{\infty}((0,+\infty), \mathbb{R})$ in such a way that $\eta(t)=0$ when $t \leq \delta / 3$ and $\eta(t)=1$ when $t \geq 2 \delta / 3$.

We define successively the functions $\tilde{\Phi}: \mathcal{M}^{\star} \times \mathcal{M} \rightarrow \mathbb{R}$ and $\Phi: \mathcal{M}^{\star} \times \mathcal{M} \rightarrow \mathbb{R}$ for every $(x, t, y) \in \mathcal{M}^{\star} \times \mathcal{M}=$ $\mathcal{M} \times(0,+\infty) \times \mathcal{M}$ by

$$
\tilde{\Phi}(x, t, y) \triangleq(1-\eta(t)) \varphi\left(\frac{d_{\mathcal{M}}(y, x)^{2}}{t^{2}}\right)+\eta(t)
$$

and

$$
\Phi(x, t, y)=\frac{\tilde{\Phi}(x, t, y)}{\int_{\mathcal{M}} \tilde{\Phi}(x, t, y) \mathrm{d} y} .
$$

We verify immediately that (7.8) holds by construction.

The second assertion (7.8) follows from the observations that the function $\tilde{\Phi}$ is bounded, that $\tilde{\Phi}(x, t, y)=$ 0 if $d_{\mathcal{M}}(x, y) \geq t$ and that for some constant $C_{1}>0$, for every $x, t \in \mathcal{M}^{*}=\mathcal{M} \times(0,+\infty)$,

$$
\int_{\mathcal{M}} \tilde{\Phi}(x, t, y) \mathrm{d} y \geq C_{1} t^{m} .
$$

For the last assertion (7.8) we observe that the map

$$
(x, t) \in \mathcal{M}^{\star} \mapsto \int_{\mathcal{M}}\left|D \Phi_{y}(x, t)\right|_{\mathcal{M}^{*}}
$$

is continuous, that if $t \geq \delta$,

$$
\int_{\mathcal{M}}\left|D \Phi_{y}(x, t)\right|_{\mathcal{M}^{*}}=0
$$

and that if $\left(x_{j}\right)_{j \in \mathbb{N}}$ is any sequence in $\mathcal{M}$ and if $\left(t_{j}\right)_{j \mathbb{N}}$ is a sequence in $(0,+\infty)$ converging to 0 , then

$$
\lim _{j \rightarrow \infty} \int_{\mathcal{M}}\left|D \Phi_{y}\left(x_{j}, t_{j}\right)\right|_{\mathcal{M}^{*}}=\int_{\mathbb{R}^{m}} \sqrt{\left|2 \varphi^{\prime}\left(|z|^{2}\right) z\right|^{2}+\left.\left|2 \varphi^{\prime}\left(|z|^{2}\right)\right| z\right|^{2}+m \varphi\left(|z|^{2}\right) \mid} \mathrm{d} z<+\infty ;
$$

hence (7.8) follows from the classical extreme value theorem for continuous functions.

Proof of proposition 7.7. We define the function $F: \mathcal{M}^{\star} \rightarrow \mathbb{R}^{v}$ by setting for every $(x, t) \in \mathcal{M}^{\star}=\mathcal{M} \times(0,+\infty)$,

$$
F(x, t) \triangleq \int_{\mathcal{M}} \Phi(x, t, y) f(y) \mathrm{d} y,
$$

where the function $\Phi \in \mathscr{C}^{\infty}\left(\mathcal{M}^{*} \times \mathcal{M}\right)$ is given by lemma 7.8.

We first observe that for every $x \in \mathcal{M}, \lim _{(y, t) \rightarrow(0,0)} F(y, t)=f(x)$ and thus assertion (7.7) holds.

For (7.7), we note that by lemma $7.8(7.8)$ we have for every $(z, s) \in \mathcal{M}^{\star}=\mathcal{M} \times(0,+\infty)$ and every $x \in \mathcal{M}$,

$$
F(z, s)-f(x)=\int_{\mathcal{M}} \Phi(z, s, y)(f(y)-f(x)) \mathrm{d} y,
$$


and thus by differentiating with respect to $(z, s)$ at the point $(x, t)$, we obtain

$$
D F(x, t)=\int_{\mathcal{M}} D \Phi_{y}(x, t)(f(y)-f(x)) \mathrm{d} y,
$$

and thus we deduce from lemma 7.8 (7.8) that

$$
|D F(x, t)|_{\mathcal{M}^{*}} \leq \underset{\mathcal{M}}{\operatorname{osc}} f \int_{\mathcal{M}}\left|D \Phi_{y}(x, t)\right|_{\mathcal{M}^{*}} \mathrm{~d} y \leq C_{1} \underset{\mathcal{M}}{\operatorname{osc} f} .
$$

For the last part (7.7), we first observe that for each $(x, t) \in \mathcal{M}^{*} \simeq \mathcal{M} \times(0,+\infty)$, we have

$$
\operatorname{dist}(F(x, t), f(\mathcal{M})) \leq|F(x, t)-f(x)| \leq \int_{\mathcal{M}} \Phi(x, t, y)|f(y)-f(x)| \mathrm{d} y .
$$

Hence we infer from lemma 7.8 (7.8),

$$
\begin{aligned}
\operatorname{dist}(F(x, t), f(\mathcal{M})) & \leq \varepsilon \int_{\mathcal{M}} \Phi(x, t, y) \mathrm{d} y+\int_{\mathcal{M}} \Phi(x, t, y)(|f(y)-f(x)|-\varepsilon)_{+} \mathrm{d} y \\
& \leq \varepsilon+C_{2} t^{m} \int_{\mathcal{M}} \frac{(|f(y)-f(x)|-\varepsilon)_{+}}{d_{\mathcal{M}}(y, x)^{2 m}} \mathrm{~d} y .
\end{aligned}
$$

We define now the set

$$
A_{\delta} \triangleq\left\{(x, t) \in \mathcal{M}^{\star} \mid \operatorname{dist}(F(x, t), f(\mathcal{M})) \geq \delta\right\}
$$

and, for each $x \in \mathcal{M}$, the quantity

$$
\tau_{\delta}(x) \triangleq \inf \left\{t \in(0,+\infty) \mid(x, t) \in A_{\delta}\right\},
$$

and we compute

$$
\mu_{\mathcal{M}^{\star}}\left(A_{\delta}\right)=\iint_{(x, t) \in A_{\delta}} \frac{1}{t^{m+1}} \mathrm{~d} x \mathrm{~d} t \leq \int_{\mathcal{M}} \int_{\tau_{\delta}(x)}^{+\infty} \frac{1}{t^{m+1}} \mathrm{~d} t \mathrm{~d} x \leq \int_{\mathcal{M}} \frac{1}{m \tau_{\delta}(x)^{m}} \mathrm{~d} x .
$$

By (7.2), we have

$$
\delta-\varepsilon=\operatorname{dist}\left(F\left(x, \tau_{\delta}(x)\right), f(\mathcal{M})\right)-\varepsilon \leq C_{2} \tau_{\delta}(x)^{m} \int_{\mathcal{M}} \frac{\left(d_{\mathcal{N}}(f(y), f(x))-\varepsilon\right)_{+}}{d_{\mathcal{M}}(y, x)^{2 m}} \mathrm{~d} y,
$$

and thus by (7.3), we conclude that

$$
\mu_{\mathcal{M}}\left(A_{\delta}\right) \leq \frac{C_{2}}{m(\delta-\varepsilon)} \iint_{\mathcal{M} \times \mathcal{M}} \frac{\left(d_{\mathcal{N}}(f(y), f(x))-\varepsilon\right)_{+}}{d_{\mathcal{M}}(y, x)^{2 m}} \mathrm{~d} y \mathrm{~d} x .
$$

\subsubsection{Merging balls}

We will use a generalization of lemma 4.3, that incorporates also a horoball, that is, a ball centered at the point at infinity of $\mathcal{M}^{*}$.

Lemma 7.9 (Merging balls and a horoball on $\mathcal{M}^{*}$ ). Given an nonnegative integer $\ell \in \mathbb{N}_{\star}$, some points $a_{1}, a_{2}, \ldots, a_{\ell} \in \mathcal{M}^{*}$, radii $r_{1}, r_{2}, \ldots, r_{\ell} \in(0,+\infty)$ and $T \in(0,+\infty)$, there exists $\ell^{\prime} \in\{0,1, \ldots, \ell\}$, points $a_{1}^{\prime}, \ldots, a_{\ell}^{\prime} \in \mathcal{M}^{*}$, radii $r_{1}^{\prime}, \ldots, r_{\ell}^{\prime}$ and $T^{\prime} \in(0, T)$ such that

(i)

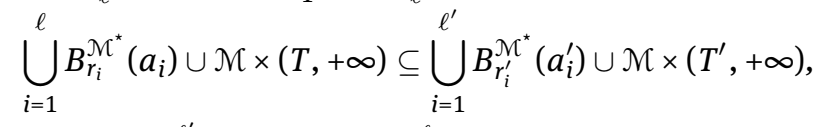

(ii) $\frac{1}{2} \ln \frac{1}{T^{\prime}}+\sum_{i=1}^{\ell^{\prime}} r_{i}^{\prime} \leq \frac{1}{2} \ln \frac{1}{T}+\sum_{i=1}^{\ell} r_{i}$ 
(iii) for every $i, j \in\left\{1,2, \ldots, \ell^{\prime}\right\}$ such that $i \neq j$, one has $\bar{B}_{r_{i}^{\prime}}^{\mathcal{\mathcal { M } ^ { * }}}\left(a_{i}^{\prime}\right) \cap \bar{B}_{r_{j}^{\prime}}^{\mathcal{\mathcal { X } ^ { * }}}\left(a_{j}^{\prime}\right)=\emptyset$,

(iv) for every $i \in\left\{1,2, \ldots, \ell^{\prime}\right\}$, one has $\bar{B}_{r_{i}}^{\mathcal{M} \mathcal{K}^{\star}}\left(a_{i}^{\prime}\right) \subseteq \mathcal{M} \times\left(0, T^{\prime}\right)$.

In contrast to lemma 4.3, we must allow in the conclusion $\ell^{\prime}=0$ if $T$ was too small at the beginning.

In order to prove lemma 7.9 we need to have good estimates on the distances between points. It turns out that this distance can be computed exactly in terms of the distance on $\mathcal{M}$.

Lemma 7.10 (Distance formula on $\left.\mathcal{M}^{*}\right)$. For every $(x, t),(y, s) \in \mathcal{M}^{*}=\mathcal{M} \times(0,+\infty)$, we have

$$
\begin{aligned}
d_{\mathcal{M}^{*}}((x, t),(y, s)) & =2 \sinh ^{-1}\left(\frac{\sqrt{d_{\mathcal{M}}(x, y)^{2}+(t-s)^{2}}}{2 \sqrt{t s}}\right) \\
& =\cosh ^{-1}\left(\frac{d_{\mathcal{M}}(x, y)^{2}+t^{2}+s^{2}}{2 t s}\right) .
\end{aligned}
$$

Proof. We consider a geodesic $y: \mathbb{R} \rightarrow \mathcal{M}$ such that $y(0)=x$ and $y\left(d_{\mathcal{M}}(x, y)\right)=y$. If $\mathbb{H}^{2}$ denotes the Poincare half-space model of the hyperbolic plane and if we define $\Phi: \mathbb{H}^{2} \rightarrow \mathcal{M}^{*}$ for $(z, r) \in \mathbb{H}^{2} \simeq \mathbb{R} \times(0,+\infty)$ by $\Phi(z, r) \triangleq(y(z), r)$, we observe that since $y$ is a geodesic, the map $\Phi$ is a local isometry and thus is globally nonexpansive from the hyperbolic plane $\mathbb{H}^{2}$ to $\mathcal{M}^{\star}$. Therefore, we have, by a classical computation of the hyperbolic distance [16, III.4 (1) and (2)], for every $s, t \in(0,+\infty)$,

$$
\begin{aligned}
d_{\mathcal{M}^{*}}((x, t),(y, s)) & =d_{\mathcal{M}^{*}}\left(\Phi(0, t), \Phi\left(d_{\mathcal{M}}(x, y), s\right)\right) \\
& \leq d^{\mathbb{H}^{2}}\left((0, t),\left(d_{\mathcal{M}}(x, y), s\right)\right) \\
& =2 \sinh ^{-1}\left(\frac{\sqrt{d_{\mathcal{M}}(x, y)^{2}+(t-s)^{2}}}{2 \sqrt{t s}}\right) \\
& =\cosh ^{-1}\left(\frac{d_{\mathcal{M}}(x, y)^{2}+t^{2}+s^{2}}{2 t s}\right) .
\end{aligned}
$$

For the converse, given $(x, t),(y, s) \in \mathcal{M}^{*}=\mathcal{M} \times(0,+\infty)$, we consider a path $y^{\star}=(y, \tau) \in \mathscr{C}^{1}\left([0,1], \mathcal{M}^{*}\right)=$ $\mathscr{C}^{1}([0,1], \mathcal{M} \times(0,+\infty))$ such that $y^{\star}(0)=(y(0), \tau(0))=(x, t)$ and $y^{\star}(1)=(y(1), \tau(1))=(y, s)$. We define the function $\xi:[0,1] \rightarrow \mathbb{R}$ for each $r \in[0,1]$ by

$$
\xi(r) \triangleq \int_{0}^{r} \sqrt{g\left(y^{\prime}(\rho)\right)} \mathrm{d} \rho .
$$

We observe than that

$$
\begin{aligned}
\int_{0}^{1} \frac{g\left(y^{\prime}(r)\right)+\left|\tau^{\prime}(r)\right|^{2}}{\tau(r)^{2}} \mathrm{~d} r & =\int_{0}^{1} \frac{\left|\xi^{\prime}(r)\right|^{2}+\left|\tau^{\prime}(r)\right|^{2}}{\tau(r)^{2}} \mathrm{~d} r \\
& \geq d^{\mathbb{H}^{2}}((0, t),(\xi(1), s)) \\
& =2 \sinh ^{-1}\left(\frac{\sqrt{\xi(1)^{2}+(t-s)^{2}}}{2 \sqrt{t s}}\right),
\end{aligned}
$$

and thus since $\xi(1) \geq d_{\mathcal{M}}(x, y)$ by definition of geodesic distance

$$
\int_{0}^{1} \frac{g\left(y^{\prime}(r)\right)+\left|\tau^{\prime}(r)\right|^{2}}{\tau(r)} \mathrm{d} r \geq 2 \sinh ^{-1}\left(\frac{\sqrt{d_{\mathcal{M}}(x, y)^{2}+(t-s)^{2}}}{2 \sqrt{t s}}\right) .
$$

As a corollary of lemma 7.10, we have the estimate.

$$
d_{\mathcal{M}^{\star}}((x, t),(y, s)) \geq 2 \sinh ^{-1}\left(\frac{|t-s|}{2 \sqrt{t s}}\right)=2 \ln \frac{|t-s|+|t+s|}{2 \sqrt{t s}}=\left|\ln \frac{t}{s}\right| .
$$


(This estimate can also be proved directly by a crude lower bound on the metric on $\mathcal{M}^{\star}$.)

Another consequence of lemma 7.10 is the completeness of the manifold $\mathcal{M}^{*}$.

Lemma 7.11. If the manifold $\mathcal{M}$ is complete, then the manifold $\mathcal{M}^{\star}$ is complete.

Proof. Assume that $\left(x_{j}, t_{j}\right)_{j \in \mathbb{N}}$ is a Cauchy sequence in $\mathcal{M}^{*}=\mathcal{M} \times(0,+\infty)$. By $(7.4)$, the sequence $\left(\ln t_{j}\right)_{j \in \mathbb{N}}$ is a Cauchy sequence in $\mathbb{R}$. By completeness of $\mathbb{R},\left(\ln t_{j}\right)_{j \in \mathbb{N}}$ converges thus to some $\tau \star \in \mathbb{R}$. In particular, the sequence $\left(t_{j}\right)_{j \in \mathbb{N}}$ converges to $t_{\star}=e^{\tau_{\star}}$ and is bounded. It follows then from lemma 7.10, that the sequence $\left(x_{j}\right)_{j \in \mathbb{N}}$ is a Cauchy sequence in the manifold $\mathcal{M}$ and thus converges to some $x_{\star}$ by completeness of $\mathcal{M}$.

We are now in position to prove our ball-merging lemma.

Proof of lemma 7.9. We proceed by induction on $\ell \in \mathbb{N}$. In the case $\ell=0$, the conclusion holds trivially. We assume now that $\ell \geq 1$ and that the conclusion holds for $\ell-1$. If the balls are all disjoint and contained in $\mathcal{M} \times(0, T)$, then the conclusion holds by setting $\ell^{\prime}=\ell$, for each $i \in\left\{1, \ldots, \ell^{\prime}\right\}, a_{i}^{\prime}=a_{i}$ and $r_{i}^{\prime}=r_{i}$, and $T^{\prime}=T$.

If the balls are not all disjoint, then up to reordering the balls we can assume that $\bar{B}_{r_{\ell-1}}^{\mathcal{\mathcal { N } ^ { * }}}\left(a_{\ell-1}\right) \cap \bar{B}_{r_{\ell}}^{\mathcal{M} \mathcal{K}^{*}}\left(a_{\ell}\right) \neq \emptyset$. By the triangle inequality, this implies that $d_{\mathcal{M}^{*}}\left(a_{\ell-1}, a_{\ell}\right) \leq r_{\ell-1}+r_{\ell}$. Since by lemma 7.11 the manifold $\mathcal{M}^{*}$ is complete and since $d_{\mathcal{M}^{*}}$ is a geodesic distance, there exists a point $\tilde{a}_{\ell-1} \in \mathcal{N}$ such that

$$
d_{\mathcal{M}}\left(\tilde{a}_{\ell-1}, a_{\ell-1}\right)=\frac{1}{2}\left(d_{\mathcal{M}^{*}}\left(a_{\ell-1}, a_{\ell}\right)+r_{\ell}-r_{\ell-1}\right),
$$

and

$$
d_{\mathcal{M}(}\left(\tilde{a}_{\ell-1}, a_{\ell}\right)=\frac{1}{2}\left(d_{\mathcal{M}^{*}}\left(a_{\ell-1}, a_{\ell}\right)+r_{\ell-1}-r_{\ell}\right) .
$$

If we define $\tilde{r}_{\ell-1} \triangleq \frac{1}{2}\left(d_{\mathcal{M}^{*}}\left(a_{\ell-1}, a_{\ell}\right)+r_{\ell-1}+r_{\ell}\right)$ we have

$$
\tilde{r}_{\ell-1} \leq r_{\ell-1}+r_{\ell} \quad \text { and } \quad B_{r_{\ell-1}}^{\mathcal{M} \mathcal{M}^{*}}\left(a_{\ell-1}\right) \cup B_{r_{\ell}}^{\mathcal{M} \mathcal{M}^{\star}}\left(a_{\ell}\right) \subseteq B_{\tilde{r}_{\ell-1}}^{\mathcal{M} \mathcal{K}^{\star}}\left(\tilde{a}_{\ell-1}\right) .
$$

We set, for $i \in\{1,2, \ldots, \ell-2\}, \tilde{a}_{i}=a_{i}$ and $\tilde{r}_{i}=r_{i}$ and $\tilde{\ell}=\ell-1$. We conclude by applying our induction hypothesis to $\tilde{\ell}, \tilde{a}_{1}, \tilde{a}_{2}, \ldots, \tilde{a}_{\tilde{\ell}}$ and $\tilde{r}_{1}, \tilde{r}_{2}, \ldots, \tilde{r}_{\tilde{\ell}}$.

If all the balls are disjoint, it can still happen that they do not all lie in $\mathcal{M} \times(0, T)$. We can assume, by a permutation of the indices, that $\bar{B}_{r_{\ell}}^{\mathcal{\mathcal { M } ^ { * }}}\left(a_{\ell}\right) \cap \mathcal{M} \times[T,+\infty) \neq \emptyset$. We define now $\tilde{T} \triangleq T e^{-2 r_{\ell}}$. By definition of the metric on $\mathcal{M}_{*}$, in view of lemma 7.10 and its consequence (7.4), this implies that

$$
B_{r_{\ell}}^{\mathcal{\mathcal { N } ^ { * }}}\left(a_{\ell}\right) \subset \mathcal{M} \times(\tilde{T},+\infty) .
$$

We conclude by setting for each $\tilde{\ell}=\ell-1$, for every $i \in\{1,2, \ldots, \ell-1\}, \tilde{a}_{i} \triangleq a_{i}$ and $\tilde{r}_{i} \triangleq r_{i}$ and applying our induction hypothesis to $\tilde{\ell}, \tilde{a}_{1}, \tilde{a}_{2}, \ldots, \tilde{a}_{\tilde{\ell}}, \tilde{r}_{1}, \tilde{r}_{2}, \ldots, \tilde{r}_{\tilde{\ell}}$ and $\tilde{T}$.

\subsubsection{Proof of the theorem}

In order to prove theorem 7.5 we need a lower bound on the measure of balls, which will be an ingredient in the control of the covering. In the proof of theorem 4.6 this bound followed directly from the transitivity of isometries of the hyperbolic space.

Lemma 7.12 (Lower bound on the volume of balls on $\mathcal{M}^{\star}$ ). There exists a constant $C>0$ such that for every $(x, t) \in \mathcal{M}^{*}=\mathcal{M} \times(0,+\infty)$,

$$
\mu_{\mathcal{M}^{*}}\left(B_{\rho}^{\mathcal{M N}^{*}}(x, t)\right) \geq C \frac{(\tanh \rho)^{m+1}}{1+(t \sinh \rho)^{m}}
$$

In particular, if $t \in(0,+\infty)$ and $\rho \in(0,+\infty)$ remain in a bounded region, we have by lemma 7.12

$$
\mu_{\mathcal{M}^{*}}\left(B_{\rho}^{\mathcal{\mathcal { M } ^ { * }}}(x, t)\right) \geq c^{\prime} \rho^{m+1} .
$$


Proof of lemma 7.12. We observe that if $s, t \in(0,+\infty), \rho \in(0,+\infty)$ and

$$
(s-t \cosh \rho)^{2} \leq \frac{(t \sinh \rho)^{2}}{2}
$$

then

If moreover, $x, y \in \mathcal{M}$ satisfy

$$
s^{2}+t^{2} \leq 2 s t \cosh \rho-\frac{(t \sinh \rho)^{2}}{2} .
$$

$$
d_{\mathcal{M}}(x, y) \leq \frac{(t \sinh \rho)^{2}}{2}
$$

then by the distance formula of lemma 7.10 ,

$$
d_{\mathcal{M}^{\star}}((y, s),(x, t))=\cosh ^{-1}\left(\frac{d_{\mathcal{M}}(x, y)^{2}+t^{2}+s^{2}}{2 t s}\right) \leq \rho .
$$

We have thus for every $(x, t) \in \mathcal{M}^{\star}=\mathcal{M} \times(0,+\infty)$ and $\rho \in(0,+\infty)$,

$$
B_{\frac{t}{\sqrt{2}}}^{\mathcal{M}} \sinh \rho(x) \times\left[t \cosh \rho-\frac{t}{\sqrt{2}} \sinh \rho, t \cosh \rho+\frac{t}{\sqrt{2}} \sinh \rho\right] \subset B_{\rho}^{\mathcal{M}^{*}}(x, t) .
$$

We infer then from the monotonicity property of measures that

$$
\begin{aligned}
& \mu_{\mathcal{M}{ }^{\star}}\left(B_{\rho}^{\mathcal{M}^{*}}(x, t)\right) \geq \int_{t \cosh \rho-\frac{t}{\sqrt{2}} \sinh \rho}^{t \cosh \rho+\frac{t}{\sqrt{2}} \sinh \rho} \frac{\mu_{\mathcal{M}}\left(B_{t / \sqrt{2} \sinh \rho}(x)\right)}{s^{m+1}} \mathrm{~d} s \\
& \geq \frac{\sqrt{2} \mu_{\mathcal{M}}\left(B_{\frac{t}{\sqrt{2}}} \sinh \rho\right.}{t^{m}(\cosh \rho)^{m+1}} \sinh \rho,
\end{aligned}
$$

by convexity of the function $s \in(0,+\infty) \mapsto s^{-(m+1)}$. Since the manifold $\mathcal{M}$ is compact, there exists a constant $C_{1}$ such that for every $x \in \mathcal{M}$ and $\sigma>0$ we have

$$
\mu_{\mathcal{M}}\left(B_{\sigma}(x)\right) \geq C_{1} \min \left(\sigma^{m}, 1\right) .
$$

It follows then from (7.5) and (7.6) that

$$
\mu_{\mathcal{M}^{*}}\left(B_{\rho}^{\mathcal{M}^{*}}(x, t)\right) \geq C_{1} \min \left(\frac{(\tanh \rho)^{m+1}}{2^{m / 2}}, \frac{\tanh \rho}{(t \cosh \rho)^{m}}\right) .
$$

The free homotopy decomposition will be made through Lipschitz-continuous maps on spheres in $\mathcal{M}^{\star}$. The next lemma ensures that the shape of these small spheres is controlled and will serve as a substitute to lemma 4.5.

Lemma 7.13 (Lower bound on the injectivity radius on $\mathcal{M}^{\star}$ ). For every $\eta>0$, there exists $T>0$ such that if $(x, t) \in \mathcal{M} \times(0, T]$, then

$$
\operatorname{inj}_{\mathcal{M}_{\star}}(x, t)=\sinh ^{-1}\left(\frac{\operatorname{inj}_{\mathcal{M}}(x)}{t}\right)
$$

and for every $\eta \in(0,1)$, the exponential map, its inverse are controlled uniformly in $\mathscr{C}^{1}$ on ball of radius $\eta \operatorname{inj}_{\mathcal{M}_{*}}(x, t)$ around $(x, t) \in \mathcal{M}$.

Proof. We observe by lemma 7.10, that for every $(x, t),(y, s) \in \mathcal{M}^{\star}=\mathcal{M} \times(0,+\infty)$,

$$
\begin{aligned}
d_{\mathcal{M}}(x, y)^{2} & =2 s t \cosh d_{\mathcal{M}^{\star}}((x, t),(y, s))-t^{2}-s^{2} \\
& =t^{2}\left(\sinh d_{\mathcal{M}^{\star}}((x, t),(y, s))\right)^{2}-\left(s-t \cosh d_{\mathcal{M}^{\star}}((x, t),(y, s))\right)^{2} \\
& \leq t^{2}\left(\sinh d_{\mathcal{M}^{\star}}((x, t),(y, s))\right)^{2} .
\end{aligned}
$$

It follows then that

$$
\operatorname{inj}_{\mathcal{M} \star}(x, t)=\sinh ^{-1}\left(\frac{\operatorname{inj}_{\mathcal{M}}(x)}{t}\right) .
$$

The bounds follow then from the compactness of $\mathcal{M}$ and the homogeneity of the metric on $\mathcal{M}^{\star}$. 
Theorem 7.5 will follow from the following statement.

Theorem 7.14 (Estimate of free homotopy decompositions by a truncated fractional energy). Let $\mathcal{M}$ and $\mathcal{N}$ be compact Riemannian manifolds. If $\varepsilon>0$ is small enough, then there is a constant $C>0$ such that for every $\lambda>0$, there exists finite sets $\mathscr{F}^{\lambda} \subset \mathscr{C}\left(\mathbb{S}^{m}, \mathcal{N}\right)$ and $\mathcal{F}_{0}^{\lambda} \subset \mathscr{C}(\mathcal{M}, \mathcal{N})$ such that any map $f \in \mathscr{C}(\mathcal{M}, \mathcal{N})$ satisfying

$$
\iint_{\mathbb{S}^{m} \times \mathbb{S}^{m}} \frac{\left(d_{\mathcal{N}}(f(y), f(x))-\varepsilon\right)_{+}}{d_{\mathcal{M}}(y, x)^{2 m}} \mathrm{~d} x \mathrm{~d} y \leq \lambda,
$$

has a free homotopy decomposition into $f_{1}, \ldots, f_{k} \in \mathscr{F}^{\lambda}$ upon $f_{0} \in \mathscr{F}_{0}^{\lambda}$ with $k \leq C \lambda$.

Proof. Let $F \in \mathscr{C}^{\infty}(\mathcal{M} \times[0,+\infty])$ be the function given by proposition 7.7, let $\mathcal{N}_{\delta}=\left\{\xi \in \mathbb{R}^{v} \mid \operatorname{dist}(y, \mathcal{N})<\delta\right\}$ and $A_{\delta}=F^{-1}\left(\mathbb{R}^{v} \backslash \mathcal{N}_{\delta}\right)$. Since the manifold $\mathcal{N}$ is embedded smoothly into $\mathbb{R}^{v}$ there exists $\delta_{\star}>0$ and a Lipschitzcontinuous retraction $\Pi: \mathcal{N}_{\delta_{*}} \rightarrow \mathcal{N}$.

By the estimate given on $F$ by proposition 7.7, there exists $\rho>0$, independent of $F$, such that if $x \in A_{\delta_{*}}$, then $B_{\rho}(x) \subset A_{\delta_{\star} / 2}$. We consider a maximal set $A \subset A_{\delta_{\star}}$ such that if for every $a, b \in A$ such that $a \neq b$, one has $d^{\mathcal{N}^{*}}(a, b) \geq 2 \rho$. This implies immediately that

$$
A_{\delta_{\star}} \subseteq \bigcup_{a \in A} B_{2 \rho}^{\mathcal{M M}^{\star}}(a)
$$

Since the balls $\left(B_{\rho}^{\mathcal{N M}^{*}}(a)\right)_{a \in A}$ are disjoint, we have by lemma 7.12 and proposition 7.7 (7.7)

$$
\begin{aligned}
\#(A \cap \mathcal{M} \times(0,1]) & \leq C_{1} \sum_{a \in A \cap \mathcal{M} \times(0,1]} \mu_{\mathcal{M}^{*}}\left(B_{\rho}^{\mathcal{M}^{*}}(a)\right) \\
& \leq C_{1} \mu_{\mathcal{\mathcal { M } ^ { * }}}\left(A_{\delta_{*} / 2}\right) \\
& \leq C_{2} \iint_{\mathcal{N} \times \mathcal{M}} \frac{\left(d_{\mathcal{N}}(f(y), f(x))-\varepsilon\right)_{+}}{d_{\mathcal{M}}(y, x)^{2 m}} \mathrm{~d} y \mathrm{~d} x \leq C_{2} \lambda .
\end{aligned}
$$

We have then

$$
A_{\delta} \subseteq \bigcup_{a \in A \cap \mathcal{M} \times(0,1]} B_{2 \rho}(a) \cup\left(e^{-2 \rho},+\infty\right) .
$$

Thanks to lemma 7.13, there exists $T \in\left(0, e^{-2 \rho}\right)$ such that if $\sigma \leq C_{2} 2 \rho \lambda$ and $(x, t) \in \mathcal{M} \times(0, T]$, then the exponential map, its inverse and their derivatives are controlled on $B_{\sigma}(x, t)$.

By lemma 7.9, there exists balls $\left(B_{\rho_{i}}^{\mathcal{M N}^{\star}}\left(a_{i}\right)\right)_{1 \leq i \leq \ell}$ and $T^{\prime} \in(0, T)$ such that in view of (7.7)

$$
A_{\delta} \subseteq \bigcup_{i=1}^{\ell} B_{\rho_{i}}^{\mathcal{M N}^{\star}}\left(a_{i}\right) \cup \mathcal{M} \times\left(T^{\prime},+\infty\right),
$$

with the estimate

$$
\frac{1}{2} \ln \frac{T}{T^{\prime}}+\sum_{i=1}^{\ell} \rho_{i} \leq 2 \rho \#\left(A \cap \mathcal{M} \times\left[0, T_{2}\right]\right) \leq 2 \rho C_{2} \lambda .
$$

This implies in particular, since $T^{\prime} \leq T$ and $\rho_{i} \geq \rho$ that

$$
\ln \frac{T}{T^{\prime}} \leq 2 \rho C_{2} \lambda \quad \ell \leq 2 C_{2} \lambda \quad \text { and } \quad \rho_{i} \leq 2 \rho C_{2} \lambda .
$$

Since $\Pi \circ F$ is Lipschitz continuous on $\mathcal{M}^{*} \backslash A_{\delta}$, it follows then that the map $f$ has a free homotopy decomposition into $\left.\Pi \circ F\right|_{\partial_{B_{\beta_{1}}}}\left(a_{1}\right), \ldots,\left.\Pi \circ F\right|_{\partial_{B_{\rho_{\ell}}}\left(a_{\ell}\right)}$ upon $\left.\Pi \circ F\right|_{\mathcal{M} \times\left\{T^{\prime}\right\}}$. Since by lemma 7.13 the exponential map is controlled in $\mathcal{M} \times(0, T]$ by a bound depending on (7.8), the Lipschitz constants of the maps $\left.\Pi \circ F\right|_{\partial_{B_{\beta_{1}}}\left(a_{1}\right)}, \ldots,\left.\Pi \circ F\right|_{\partial_{B_{\rho_{\ell}}}\left(a_{\ell}\right)}$ upon $\left.\Pi \circ F\right|_{\mathcal{M} \times\left\{T^{\prime}\right\}}$ are bounded independently of $f$ and the geometry of their domains are controlled by quantities depending only on $\lambda$, and thus by Ascoli's compactness theorem the maps $\left.\Pi \circ F\right|_{\partial_{B_{\rho_{1}}}\left(a_{1}\right)}, \ldots,\left.\Pi \circ F\right|_{\partial_{B_{\rho_{\ell}}}\left(a_{\ell}\right)}$ and $\left.\Pi \circ F\right|_{\mathcal{N} \times\left\{T^{\prime}\right\}}$ are respectively homotopic to maps taken in some finite sets $\mathscr{F}^{\lambda} \subset \mathscr{C}\left(\mathbb{S}^{m}, \mathcal{N}\right)$ and $\mathscr{F}_{0}^{\lambda} \subset \mathscr{C}(\mathcal{M}, \mathcal{N})$. 


\subsection{Estimates of free homotopy decompositions by a scaled gap potential}

We obtain a version of theorem 7.5 that scales optimally with respect to $\varepsilon$, which generalizes theorem 5.4 to a general domain $\mathcal{M}$.

Theorem 7.15 (Estimate of free homotopy decompositions by a scaled gap potential). Let $\mathcal{N}$ and $\mathcal{N}$ be compact Riemannian manifolds. If $\operatorname{dim} \mathcal{M}=m \geq 2$, then there are constants $C>0$ and $\varepsilon_{0}>0$ such that for every $\lambda>0$, there exists finite sets $\mathscr{F}^{\lambda} \subset \mathscr{C}\left(\mathbb{S}^{m}, \mathcal{N}\right)$ and $\mathcal{F}_{0}^{\lambda} \subset \mathscr{C}(\mathcal{M}, \mathcal{N})$ such that if $\varepsilon \in\left(0, \varepsilon_{0}\right)$, then any $f \in \mathscr{C}(\mathcal{M}, \mathcal{N})$ satisfying

$$
\iint_{\substack{(x, y) \in \mathcal{M} \times \mathcal{M} \\ d_{\mathcal{N}}(f(y), f(x))>\varepsilon}} \frac{\varepsilon^{m}}{d_{\mathcal{M}}(y, x)^{2 m}} \mathrm{~d} x \mathrm{~d} y \leq \lambda,
$$

has a free homotopy decomposition into $f_{1}, \ldots, f_{k} \in \mathscr{F}^{\lambda}$ upon $f_{0} \in \mathscr{F}_{0}^{\lambda}$ with $k \leq C \lambda$.

Theorem 7.15 will be deduced from theorem 7.5 thanks to proposition 5.2, proposition 5.5 and the counterpart of lemma 5.3.

Lemma 7.16 (Covering pairs of a compact manifold). If $\mathcal{M}$ is a connected compact manifold of dimension $m$, then there exists open sets $A_{1}, \ldots, A_{\ell} \subset \mathcal{M}$ such that for each $i \in\{1, \ldots, \ell\}$, the set $\bar{A}_{i}$ is diffeomorphic to the closed ball $\mathbb{B}_{1} \subset \mathbb{R}^{m}$ and such that

$$
\mathcal{M} \times \mathcal{M} \subseteq \bigcup_{i=1}^{\ell} A_{i} \times A_{i} .
$$

Proof. Since $\mathcal{M}$ is connected, for every $x, y \in \mathcal{M}$, there exists an open set $A \subset \mathcal{M}$ such that $\bar{A}$ is diffeomorphic to the closed ball $\overline{\mathbb{B}}^{m} \subset \mathbb{R}^{m}$ and such that $\{x, y\} \subset A$ and therefore $(x, y) \in A \times A$. The conclusion then follows by compactness of $\mathcal{M} \times \mathcal{M}$.

Proof of theorem 7.15. This follows from theorem 7.5, proposition 5.5 and lemma 7.16.

\subsection{Estimates on the induced cohomology homomorphism}

As for maps from the sphere, it is possible to obtain linear bounds for cohomology invariants of mappings $f: \mathcal{N} \rightarrow \mathcal{N}$

If $f: \mathcal{M} \rightarrow \mathcal{N}$ is a smooth map, then its pullback $f^{\star}$ induces a homomorphism $f^{\star}: H_{\mathrm{dR}}^{\star}(\mathcal{N}) \rightarrow H_{\mathrm{dR}}^{\star}(\mathcal{N})$ on the de Rham cohomology [31, §10]. Indeed, if $\omega \in \mathscr{C}^{\infty}\left(\bigwedge^{\ell} \mathcal{N}\right)$ and $d \omega=0$, then $d\left(f^{\star} \omega\right)=0$ and moreover if $\theta \in \mathscr{C}^{\infty}\left(\bigwedge^{\ell-1} \mathcal{N}\right)$, then $f^{\star}(\omega+d \theta)=f^{\star} \omega+d\left(f^{\star} \theta\right)$. This induced homomorphism is invariant under homotopies. Cohomology induced homomorphism are linear operator on the finite dimensional de Rham cohomology groups; this allows one to define a norm $\|f\|_{\star}$ on cohomology induced homomorphisms $f_{\star}$.

If $\mathcal{M}=\mathbb{S}^{m}$, then $H_{\mathrm{dR}}^{\ell}\left(\mathbb{S}^{m}\right) \neq\{0\}$ if and only if $m=\ell$; the induced cohomology homomorphism $f^{\star}$ is then completely described by the Hurewicz homomorphism.

The following theorem generalizes the estimate for the Hurewicz homomorphism theorem 6.1 to the cohomology homomorphism.

Theorem 7.17 (Estimate of the cohomology induced homomorphism by a scaled gap potential). Let $\mathcal{N}$ and $\mathcal{N}$ be a compact Riemannian manifolds. If $\operatorname{dim} \mathcal{M} \geq 2$ and if $\varepsilon>0$ is small enough, then for every $f \in \mathscr{C}^{1}(\mathcal{M}, \mathcal{N})$, one has

$$
\left\|f^{\star}\right\| \leq C \quad \iint_{\substack{(x, y) \in \mathbb{S}^{m} \times \mathbb{S}^{m} \\ d_{\mathcal{N}}(f(y), f(x))>\varepsilon}} \frac{\varepsilon^{m}}{d_{\mathcal{M}}(y, x)^{2 m}} \mathrm{~d} x \mathrm{~d} y .
$$


Proof. Since $\mathcal{M}$ and $\mathcal{N}$ are compact, the dimension of the de Rham cohomology is finite and thus, in view of the Poincaré duality [31, §10.4], it suffices to estimate for every $\ell \in\{0, \ldots, m\}$, for every $\omega \in \mathscr{C}^{\infty}\left(\bigwedge^{\ell} \mathcal{N}\right)$ and every $\theta \in \mathscr{C}^{\infty}\left(\bigwedge^{m-\ell} \mathcal{M}\right)$ such that $d \omega=0$ and $d \theta=0$ the quantity

$$
\int_{\mathcal{M}} f^{\star} \omega \wedge \theta
$$

As before, since $\mathcal{N}$ is a compact embedded submanifold of $\mathbb{R}^{v}$, there exists an open set $U \subset \mathbb{R}^{v}$ with $\mathcal{N} \subset U$ and a smooth retraction $\Pi \in \mathscr{C}^{\infty}(U, \mathcal{N})$. We fix a function $\eta \in \mathscr{C}^{\infty}\left(\mathbb{R}^{v}, \bigwedge^{0} \mathbb{R}^{v}\right)$ such that $\eta=1$ on $\mathcal{N}$ and $\operatorname{supp} \eta \subset U$ and a canonical projection map $P: \mathcal{M} \times[0,+\infty) \rightarrow \mathcal{M}$ defined for each $(x, t) \in \mathcal{M} \times[0,+\infty)$ by $P(x, t) \triangleq x$.

We consider the map $F: \mathcal{M}^{*} \rightarrow \mathbb{R}^{v}$ given by proposition 7.7. By the Stokes-Cartan formula we have

$$
\int_{\mathcal{M}} f^{\star} \omega \wedge \theta=\int_{\mathcal{M}} F^{\star}\left(\eta \wedge \Pi^{\star} \omega\right) \wedge P^{\star} \theta=\int_{\mathcal{M}^{\star}} d\left(F^{\star}\left(\eta \wedge \Pi^{\star} \omega\right) \wedge P^{\star} \theta\right) .
$$

We compute then, since $d \omega=0$ and $d \theta=0$,

$$
d\left(F^{\star}\left(\eta \wedge \Pi^{\star} \omega\right) \wedge P^{\star} \theta\right)=F^{\star}\left(d \eta \wedge \Pi^{\star} \omega\right) \wedge P^{\star} \theta,
$$

and we conclude by proposition 7.7 that

$$
\begin{aligned}
\left|\int_{\mathcal{M}} f^{\star} \omega \wedge \theta\right| \leq & C_{1}\|D F\|_{L^{\infty}\left(\mathcal{M}^{\star}\right)}^{\ell+1}\|d \eta\|_{L^{\infty}\left(\mathbb{R}^{v}\right)}\|\omega\|_{L^{\infty}(\mathcal{N})}\|\theta\|_{L^{\infty}(\mathcal{M})} \\
& \mu_{\mathcal{M}^{*}}\left(\left\{(x, t) \in \mathcal{M}^{\star} \mid \operatorname{dist}(F(x, t), \mathcal{N}) \geq \delta\right\}\right) \\
& \leq C_{2}\|\omega\|_{L^{\infty}(\mathcal{N})}\|\theta\|_{L^{\infty}(\mathcal{M})} \iint_{\mathcal{M} \times \mathcal{M}} \frac{\left(d_{\mathcal{N}}(f(y), f(x))-\varepsilon\right)_{+}}{d_{\mathcal{M}}(y, z)^{2 m}} \mathrm{~d} y \mathrm{~d} x .
\end{aligned}
$$

By considering all admissible $\ell \in\{0, \ldots, m\}$, every $\omega \in \mathscr{C}^{\infty}\left(\bigwedge^{\ell} \mathcal{N}\right)$ and every $\theta \in \mathscr{C}^{\infty}\left(\bigwedge^{m-\ell} \mathcal{N}\right)$ such that $d \omega=0$ and $d \theta=0$, we conclude that

$$
\left\|f^{\star}\right\| \leq C_{3} \iint_{\mathcal{M} \times \mathcal{M}} \frac{(|f(y)-f(z)|-\varepsilon)_{+}}{d_{\mathcal{M}}(y, z)^{2 m}} \mathrm{~d} y \mathrm{~d} z .
$$

By proposition 5.1, proposition 5.5 and lemma 7.16, the conclusion then follows.

\section{Further problems}

A first question that remains open at the end of the present work is whether estimates with optimal scaling can be proved when $m=1$.

Open problem 1. Does theorem 5.8 hold for $m=1$ ?

A variant of this problem would be to obtain estimates with optimal scaling on the Hurewicz homomorphism when $m=1$.

Open problem 2. Does theorem 6.2 hold when $m=1$ ?

The problem is already open for maps for the degree of maps from the circle $\mathbb{S}^{1}$ to the circle $\mathbb{S}^{1}$, that is when $\mathcal{N}=\mathbb{S}^{1}$ (see [43]). It is striking that the present work and NGUYÊN Hoài-Minh followed quite different strategies of proof but encountered the same restriction that $m>1$.

The solution of theorem 5.8 and open problem 2 could be connected to the following more technical question of scaling of truncated integrals. 
Open problem 3. If $p \in[0,1)$ and $m \in \mathbb{N}$, does there exists a constant $C>0$ such that for every convex set $\Omega \subset \mathbb{R}^{m}$ and for every map $f: \Omega \rightarrow \mathbb{R}^{\nu}$, if $\delta<\varepsilon$, then

$$
\iint_{\substack{(x, y) \in \Omega \times \Omega \\
d_{\mathcal{N}}(f(y), f(x)) \geq \varepsilon}} \frac{\left(d_{\mathcal{N}}(f(y), f(x))-\varepsilon\right)^{p}}{|y-x|^{2 m}} \mathrm{~d} x \mathrm{~d} y \leq C\left(\frac{\delta}{\varepsilon}\right)^{m-p} \iint_{\begin{array}{c}
(x, y) \in \Omega \times \Omega \\
d_{\mathcal{N}}(f(y), f(x)) \geq \delta
\end{array}} \frac{\left(d_{\mathcal{N}}(f(y), f(x))-\delta\right)^{p}}{|y-x|^{2 m}} \mathrm{~d} x \mathrm{~d} y ?
$$

By proposition 5.1, the answer is positive when $p \in[1,+\infty)$ and when $p \in[0,1)$, the estimate holds with an exponent $m-1$ instead of $m-p$.

As we have mentioned in the introduction, for every $\lambda>0$, there exists a finite collection of maps $\mathscr{F}^{\lambda}$ such that every $f \in\left(\mathscr{C} \cap W^{1,1}\right)\left(\mathbb{S}^{1}, \mathcal{N}\right)$ is homotopic to some map in $\mathscr{F}^{\lambda}$. The proof is done by showing that $f$ is homotopic to a constant speed reparametrization and reduces thus the problem to Lipschitz-continuous maps to which the Ascoli theorem applies. This raises naturally the question about $W^{m, 1}\left(\mathbb{S}^{m}, \mathcal{N}\right)$.

Open problem 4. Is it true that if for $m \geq 2$, if $\lambda>0$, then the maps $f \in\left(\mathscr{C} \cap W^{m, 1}\right)\left(\mathbb{S}^{m}, \mathcal{N}\right)$, such that $\int_{\mathbb{S}^{m}}\left|D^{m} f\right| \leq \lambda$ belong to finitely many homotopy classes?

Finally, for maps from $\mathbb{S}^{2 n-1}$ to $\mathbb{S}^{n}$, the Hopf invariant can be computed through formulas that yield Rivière's estimate (1.6). The next logical step would be to obtain corresponding estimates in fractional Sobolev spaces.

Open problem 5. If $n$ is even and $p>2 n-1$, does there exists a constant such that every $f \in\left(W^{s, p} \cap\right.$ $\mathscr{C})\left(\mathbb{S}^{2 n-1}, \mathbb{S}^{n}\right)$, with $s=(2 n-1) / p$ satisfies

$$
\left|\operatorname{deg}_{H}(f)\right| \leq C\left(\iint_{\mathbb{S}^{2 n-1} \times \mathbb{S}^{2 n-2}} \frac{d_{\mathcal{N}}(f(y), f(x))^{p}}{|y-x|^{2(2 n-1)}} \mathrm{d} y \mathrm{~d} x\right)^{1+\frac{1}{2 n-1}} ?
$$

The question has been settled partially by Armin SCHIKORRA and the author when $p$ is close to $2 n-1$ [53].

A further question would be to obtain gap potential estimates:

Open problem 6. Does there exists a constant such that if $f \in \mathscr{C}\left(\mathbb{S}^{2 n-1}, \mathbb{S}^{n}\right)$,

$$
\left|\operatorname{deg}_{H}(f)\right| \leq C\left(\iint_{\substack{y, z \in \mathbb{S}^{2 n-1} \\|f(y)-f(z)| \geq \varepsilon}} \frac{\mathrm{d} y \mathrm{~d} z}{|y-z|^{2(2 n-1)}}\right)^{1+\frac{1}{2 n-1}} ?
$$

The exponent in open problem 5 and open problem 6 is justified by the following lower bound.

Proposition 8.1. If $n \in \mathbb{N}$ is even, then there exists a sequence of maps $\left(f_{k}\right)_{k \in \mathbb{N}}$ in $\mathscr{C}\left(\mathbb{S}^{2 n-1}, \mathbb{S}^{n}\right)$ such that $\left|\operatorname{deg}_{H} f_{k}\right| \rightarrow \infty$ and

$$
\iint_{\substack{x, y \in \mathbb{S}^{2 n-1} \\\left|f_{k}(y)-f_{k}(x)\right| \geq \varepsilon}} \frac{\mathrm{d} y \mathrm{~d} x}{|y-x|^{2(2 n-1)}} \leq C\left(\operatorname{deg}_{H}\left(f_{k}\right)\right)^{1-\frac{1}{2 n}} .
$$

Proof. The proof follow Tristan Riviène's proof [49, lemma III.1]. We construct for every $k \in \mathbb{N}$, the map $f_{k}=\varphi_{k} \circ \varphi$ where $\psi: \mathbb{S}^{2 n-1} \rightarrow \mathbb{S}^{n}$ has a nontrivial Hopf degree and $\varphi_{k}: \mathbb{S}^{n} \rightarrow \mathbb{S}^{n}$ has the property that $\left|D \varphi_{k}\right| \leq k^{1 / n}$ on $\mathbb{S}^{n}$ and its Brouwer degree satisfies $\operatorname{deg}\left(\varphi_{k}\right)=k$. It follows that $\left|D f_{k}\right| \leq k^{1 / n}$ and $\operatorname{deg}_{H}\left(f_{k}\right)=k^{2}$. Moreover we have

$$
\iint_{\substack{x, y \in \mathbb{S}^{2 n-1} \\\left|f_{k}(y)-f_{k}(x)\right| \geq \varepsilon}} \frac{\mathrm{d} y \mathrm{~d} x}{|y-x|^{2(2 n-1)}} \leq k^{2-\frac{1}{n}} \simeq\left(\operatorname{deg}_{H}\left(f_{k}\right)\right)^{1-\frac{1}{2 n}} .
$$

A strategy that follows the proof of theorem 4.6 constructs for a given $f \in \mathscr{C}\left(\mathbb{S}^{2 n-1}, \mathbb{S}^{n}\right)$ a decomposition into $g_{i}: \mathbb{S}^{2 n-1} \rightarrow \mathbb{S}^{n}$, with $1 \leq i \leq k$, which have a Lipschitz constant controlled by $C_{1} \sinh \rho_{i}$, with $\sum_{i=1}^{k} \rho_{i} \leq C_{2} \lambda$. 
It follows then by Rivière's bound (1.7) and by convexity that

$$
\operatorname{deg}_{H}(f)=\sum_{i=1}^{k} \operatorname{deg}_{H}\left(g_{i}\right) \leq C_{3} \sum_{i=1}^{k}\left(C_{1} \sinh \rho_{i}\right)^{2 n} \leq C_{4} \sinh C_{5} \lambda,
$$

which is quite far from the estimate proposed in open problem 6.

Acknowledgement: J. Van Schaftingen was supported by the Mandat d'Impulsion Scientifique F.4523.17, "Topological singularities of Sobolev maps" of the Fonds de la Recherche Scientifique-FNRS

\section{References}

[1] L. V. Ahlfors, Möbius transformations in several dimensions, Ordway Professorship Lectures in Mathematics, University of Minnesota School of Mathematics, Minneapolis, Minn., 1981.

[2] H. J. Baues, Obstruction theory on homotopy classification of maps, Lecture Notes in Mathematics, vol. 628, Springer, BerlinNew York, 1977.

[3] R. Bott and L. W. Tu, Differential forms in algebraic topology, Graduate Texts in Mathematics, vol. 82, Springer, New YorkBerlin, 1982.

[4] J. Bourgain, H. Brezis, and P. Mironescu, Lifting, degree, and distributional Jacobian revisited, Comm. Pure Appl. Math. 58 (2005), no. 4, 529-551, DOI 10.1002/cpa.20063.

[5] J. Bourgain, H. Brezis, and Nguyen H.-M., A new estimate for the topological degree, C. R. Math. Acad. Sci. Paris 340 (2005), no. 11, 787-791, DOI 10.1016/j.crma.2005.04.007.

[6] A. Boutet de Monvel-Berthier, V. Georgescu, and R. Purice, A boundary value problem related to the Ginzburg-Landau model, Comm. Math. Phys. 142 (1991), no. 1, 1-23.

[7] H. Brezis, Functional analysis, Sobolev spaces and partial differential equations, Universitext, Springer, New York, N.Y., 2011.

[8] H. Brezis and Li Y. Y., Topology and Sobolev spaces, C. R. Acad. Sci. Paris Sér. I Math. 331 (2000), no. 5, 365-370, DOI 10.1016/S0764-4442(00)01656-6.

[9] H. Brezis and Li Y. Y., Topology and Sobolev spaces, J. Funct. Anal. 183 (2001), no. 2, 321-369, DOI 10.1006/jfan.2000.3736.

[10] H. Brezis and Nguyen H.-M., On a new class of functions related to VMO, C. R. Math. Acad. Sci. Paris 349 (2011), no. 3-4, 157-160, DOI 10.1016/j.crma.2010.11.026.

[11] H. Brezis and L. Nirenberg, Degree theory and BMO. I: Compact manifolds without boundaries, Selecta Math. (N.S.) 1 (1995), no. 2, 197-263, DOI 10.1007/BF01671566.

[12] Chen J. and Li Y., Homotopy classes of harmonic maps of the stratified 2-spheres and applications to geometric flows, Adv. Math. 263 (2014), 357-388, DOI 10.1016/j.aim.2014.07.001.

[13] O. Druet, E. Hebey, and F. Robert, Blow-up theory for elliptic PDEs in Riemannian geometry, Mathematical Notes, vol. 45, Princeton University Press, Princeton, N.J., 2004.

[14] F. Duzaar and E. Kuwert, Minimization of conformally invariant energies in homotopy classes, Calc. Var. Partial Differential Equations 6 (1998), no. 4, 285-313, DOI 10.1007/s005260050092.

[15] B. Dyda, A fractional order Hardy inequality, Illinois J. Math. 48 (2004), no. 2, 575-588.

[16] W. Fenchel, Elementary geometry in hyperbolic space, De Gruyter Studies in Mathematics, vol. 11, de Gruyter, Berlin, 1989.

[17] W. H. Fleming, Flat chains over a finite coefficient group, Trans. Amer. Math. Soc. 121 (1966), 160-186, DOI 10.2307/1994337.

[18] A. Gastel and A. J. Nerf, Minimizing sequences for conformally invariant integrals of higher order, Calc. Var. Partial Differential Equations 47 (2013), no. 3-4, 499-521, DOI 10.1007/s00526-012-0525-0.

[19] P. Goldstein and P. Hajłasz, Sobolev mappings, degree, homotopy classes and rational homology spheres, J. Geom. Anal. 22 (2012), no. 2, 320-338, DOI 10.1007/s12220-010-9194-4.

[20] M. Gromov, Quantitative homotopy theory, Prospects in mathematics (Princeton, N.J., 1996), Amer. Math. Soc., Providence, R.I., 1999, pp. 45-49.

[21] M. Gromov, Metric structures for Riemannian and non-Riemannian spaces, translated by S. M. Bates, with appendices by M. Katz, P. Pansu, and S. Semmes, Progress in Mathematics, vol. 152, Birkhäuser, Boston, Mass., 1999.

[22] P. Hajłasz, T. Iwaniec, J. Malý, and J. Onninen, Weakly differentiable mappings between manifolds, Mem. Amer. Math. Soc. 192 (2008), no. 899, DOI 10.1090/memo/0899.

[23] Hang F. and Lin F., Topology of Sobolev mappings, Math. Res. Lett. 8 (2001), no. 3, 321-330, DOI 10.4310/MRL.2001.v8.n3.a8.

[24] Hang F. and Lin F., Topology of Sobolev mappings. II, Acta Math. 191 (2003), no. 1, 55-107, DOI 10.1007/BF02392696.

[25] Hang F. and Lin F., Topology of Sobolev mappings. III, Comm. Pure Appl. Math. 56 (2003), no. 10, 1383-1415, DOI 10.1002/cpa.10098.

[26] Hang F. and Lin F., Topology of Sobolev mappings. IV, Discrete Contin. Dyn. Syst. 13 (2005), no. 5, 1097-1124, DOI 10.3934/dcds.2005.13.1097. 
[27] A. Hatcher, Algebraic topology, Cambridge University Press, Cambridge, 2002.

[28] S.-t. Hu, Homotopy theory, Pure and Applied Mathematics, Vol. VIII, Academic Press, New York-London, 1959.

[29] R. L. Jerrard, Lower bounds for generalized Ginzburg-Landau functionals, SIAM J. Math. Anal. 30 (1999), no. 4, 721-746, DOI 10.1137/S0036141097300581.

[30] E. Kuwert, A compactness result for loops with an $H^{1 / 2}$-bound, J. Reine Angew. Math. 505 (1998), 1-22, DOI 10.1515/crll.1998.117.

[31] J. M. Lee, Manifolds and differential geometry, Graduate Studies in Mathematics, vol. 107, American Mathematical Society, Providence, R.I., 2009.

[32] W. Magnus, A. Karrass, and D. Solitar, Combinatorial group theory: Presentations of groups in terms of generators and relations, Interscience Publishers (John Wiley \& Sons), New York-London-Sydney, 1966.

[33] P. Mironescu, Sobolev maps on manifolds: degree, approximation, lifting, Perspectives in nonlinear partial differential equations, Contemp. Math., vol. 446, Amer. Math. Soc., Providence, R.I., 2007, pp. 413-436, DOI 10.1090/conm/446/08642.

[34] T. Müller, Compactness for maps minimizing the n-energy under a free boundary constraint, Manuscripta Math. 103 (2000), no. 4, 513-540, DOI 10.1007/PL00005864.

[35] Nguyen H.-M., Some new characterizations of Sobolev spaces, J. Funct. Anal. 237 (2006), no. 2, 689-720, DOI 10.1016/j.jfa.2006.04.001.

[36] Nguyen H.-M., Г-convergence and Sobolev norms, C. R. Math. Acad. Sci. Paris 345 (2007), no. 12, 679-684, DOI 10.1016/j.crma.2007.11.005.

[37] Nguyen H.-M., Optimal constant in a new estimate for the degree, J. Anal. Math. 101 (2007), 367-395, DOI 10.1007/s11854-0070014-0.

[38] Nguyen H.-M., Further characterizations of Sobolev spaces, J. Eur. Math. Soc. (JEMS) 10 (2008), no. 1, 191-229, DOI 10.4171/JEMS/108.

[39] H.-M. Nguyen, Inequalities related to liftings and applications, C. R. Math. Acad. Sci. Paris 346 (2008), no. 17-18, 957-962, DOI 10.1016/j.crma.2008.07.026.

[40] Nguyen H.-M., Г-convergence, Sobolev norms, and BV functions, Duke Math. J. 157 (2011), no. 3, 495-533, DOI 10.1215/00127094-1272921.

[41] H.-M. Nguyen, Some inequalities related to Sobolev norms, Calc. Var. Partial Differential Equations 41 (2011), no. 3-4, 483-509, DOI 10.1007/s00526-010-0373-8.

[42] Nguyen H.-M., Estimates for the topological degree and related topics, J. Fixed Point Theory Appl. 15 (2014), no. 1, 185-215, DOI 10.1007/s11784-014-0182-3.

[43] Nguyen H.-M., A refined estimate for the topological degree, C. R. Math. Acad. Sci. Paris 355 (2017), no. 10, 1046-1049, DOI 10.1016/j.crma.2017.10.007.

[44] H.-M. Nguyen, A. Pinamonti, M. Squassina, and E. Vecchi, New characterizations of magnetic Sobolev spaces, Adv. Nonlinear Anal. 7 (2018), no. 2, 227-245, DOI 10.1515/anona-2017-0239.

[45] T. H. Parker, What is... a bubble tree?, Notices Amer. Math. Soc. 50 (2003), no. 6, 666-667.

[46] P. Petersen, Riemannian geometry, 3rd ed., Graduate Texts in Mathematics, vol. 171, Springer, Cham, 2016.

[47] M. Petrache and T. Rivière, Global gauges and global extensions in optimal spaces, Anal. PDE 7 (2014), no. 8, 1851-1899, DOI 10.2140/apde.2014.7.1851.

[48] M. Petrache and J. Van Schaftingen, Controlled singular extension of critical trace Sobolev maps from spheres to compact manifolds, Int. Math. Res. Not. IMRN 12 (2017), 3467-3683, DOI 10.1093/imrn/rnw109.

[49] T. Rivière, Minimizing fibrations and p-harmonic maps in homotopy classes from $\mathbb{S}^{3}$ into $\mathbb{S}^{2}$, Comm. Anal. Geom. 6 (1998), no. 3, 427-483, DOI 10.4310/CAG.1998.v6.n3.a2.

[50] J. Sacks and K. Uhlenbeck, The existence of minimal immersions of 2-spheres, Ann. of Math. (2) 113 (1981), no. 1, 1-24, DOI $10.2307 / 1971131$.

[51] E. Sandier, Lower bounds for the energy of unit vector fields and applications, J. Funct. Anal. 152 (1998), no. 2, 379-403, DOI 10.1006/jfan.1997.3170.

[52] E. Sandier and S. Serfaty, Vortices in the magnetic Ginzburg-Landau model, Progress in Nonlinear Differential Equations and their Applications, vol. 70, Birkhäuser, Boston, Mass., 2007.

[53] A. Schikorra and J. Van Schaftingen, An estimate of the Hopf degree of fractional Sobolev mappings. arXiv:1904.12549.

[54] R. Schoen and J. Wolfson, Minimizing area among Lagrangian surfaces: the mapping problem, J. Differential Geom. 58 (2001), no. 1, 1-86, DOI 10.4310/jdg/1090348282.

[55] J.-P. Serre, Homologie singulière des espaces fibrés. Applications, Ann. of Math. (2) 54 (1951), 425-505, DOI 10.2307/1969485.

[56] Toda H., Composition methods in homotopy groups of spheres, Annals of Mathematics Studies, No. 49, Princeton University Press, Princeton, N.J., 1962.

[57] B. White, Infima of energy functionals in homotopy classes of mappings, J. Differential Geom. 23 (1986), no. 2, 127-142, DOI $10.4310 / j d g / 1214440023$.

[58] J. H. C. Whitehead, An expression of Hopf's invariant as an integral, Proc. Nat. Acad. Sci. U. S. A. 33 (1947), 117-123.

[59] M. Willem, Functional analysis: Fundamentals and applications, Cornerstones, Birkhäuser/Springer, New York, N.Y., 2013. 